INEEL/EXT-2000-01097

August 2000

\title{
Tank Farm WM-182 and WM-183 Heel Slurry Samples PSD Results
}

T. A. Batcheller

G. M. Huestis

BECHTEL BWXTIDAHO, L L C 


\title{
Tank Farm WM-182 and WM-183 Heel Slurry Samples PSD Results
}

\author{
T. A. Batcheller \\ G. M. Huestis
}

Published August 2000

Idaho National Engineering and Environmental Laboratory Idaho Falls, Idaho 83415

\author{
Prepared for the \\ U.S. Department of Energy \\ Assistant Secretary for \\ Environmental Management \\ Under DOE Idaho Operations Office \\ Contract DE-AC07-99ID13727
}




\section{SUMMARY}

Particle size distribution (PSD) analysis of INTEC Tank Farm WM-182 and WM-183 heel slurry samples were performed using a modified Horiba LA-300 PSD analyzer at the RAL facility. There were two types of testing performed: typical PSD analysis, and settling rate testing.

Although the heel slurry samples were obtained from two separate vessels, the particle size distribution results were quite similar. The slurry solids were from approximately a minimum particle size of $0.5 \mu \mathrm{m}$ to a maximum of $230 \mu \mathrm{m}$-with about $90 \%$ of the material between 2-to- $133 \mu \mathrm{m}$, and the cumulative $50 \%$ value at approximately $20 \mu \mathrm{m}$. This testing also revealed that high frequency sonication with an ultrasonic element may break-up larger particles in the WM-182 and WM-183 tank farm heel slurries. This finding represents useful information regarding ultimate tank heel waste processing.

Settling rate testing results were also fairly consistent with material from both vessels in that it appears that most of the mass of solids settle to an agglomerated, yet easily redispersed layer at the bottom. A dispersed and suspended material remained in the "clear" layer above the settled layer after about one-half an hour of settling time. This material had a statistical mode of approximately $5 \mu \mathrm{m}$ and a maximum particle size of $30 \mu \mathrm{m}$. 


\section{CONTENTS}

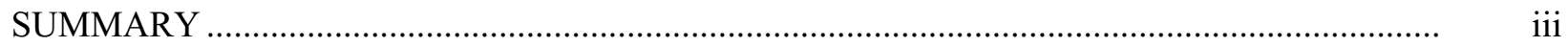

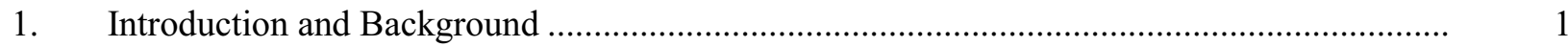

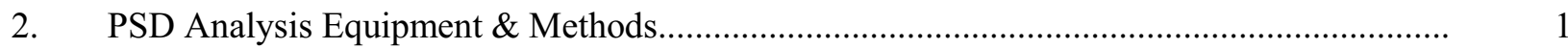

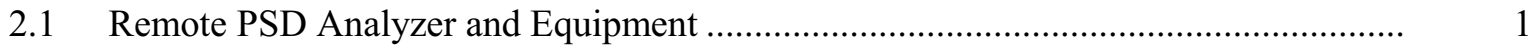

2.2 Analyzer Performance Check with Standards ......................................................... 2

2.3 Tank Farm Samples, PSD Analysis Method and Testing …...................................... 2

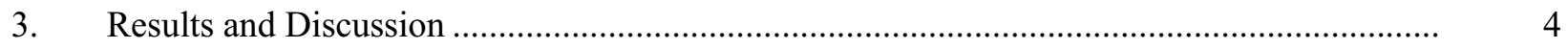

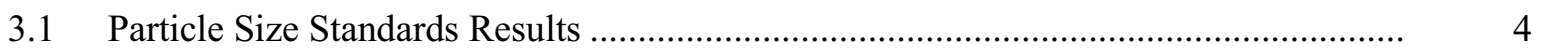

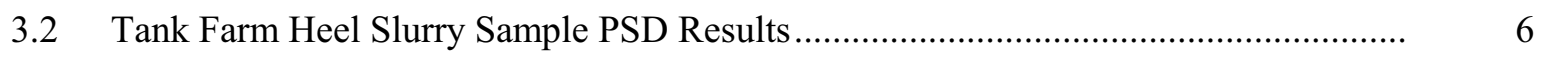

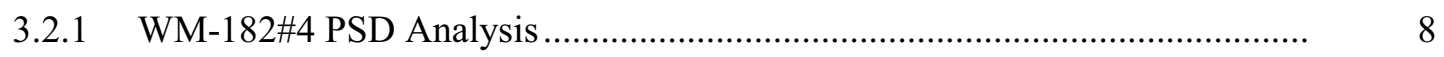

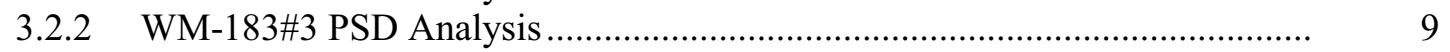

3.2.3 WM-183 Composite A PSD Analysis......................................................... 10

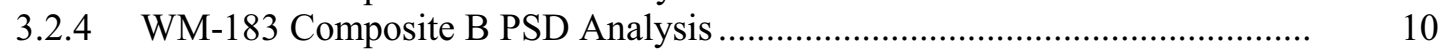

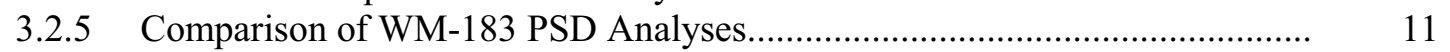

3.2.6 Comparison Between WM-182 and WM-183 PSD Analyses......................... 12

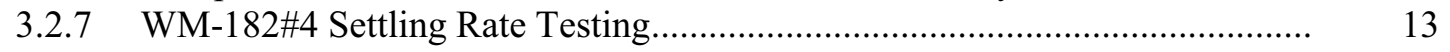

3.2.8 WM-183 Composite A Settling Rate Testing ….......................................... 14

3.2.9 WM-183 Composite B Settling Rate Testing ............................................... 16

3.2.10 Comparison Between WM-182 and WM-183 Settling Rate Testing Results.. $\quad 16$

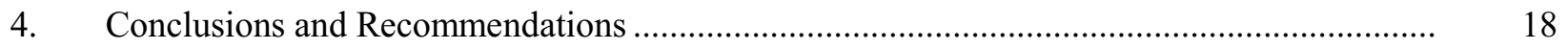

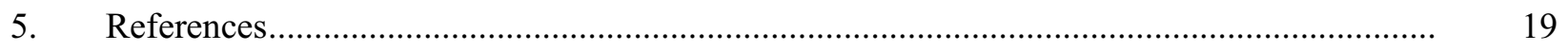

Appendix —A-1 Particle Size Standards Results Data ........................................................... A-2

Appendix -A-2 Tank Farm Slurry Sample PSD Results Data .............................................. A-7

Appendix —A-3 Settling Rate Testing Photographs …...................................................... A-32 


\section{FIGURES}

1. $\quad 35 \mu \mathrm{m}$ garnet particle size standard PSD analyses results w/SEM photomicrograph........... 4

2. $\quad$ Mixture of Coulter standards; 2.1 and $20.8 \mu \mathrm{m}$ microspheres ......................................... 5

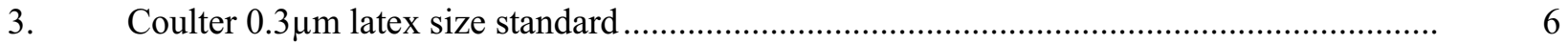

4. WM-182 \#4 PSD Analyses; avg. PSD for non sonicated samples...................................... 8

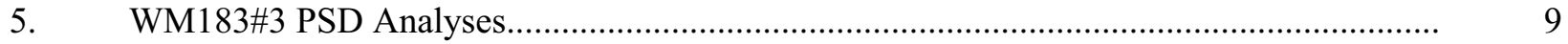

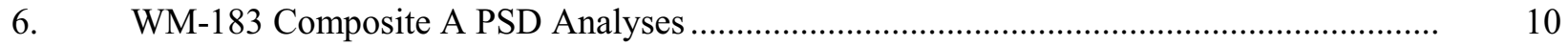

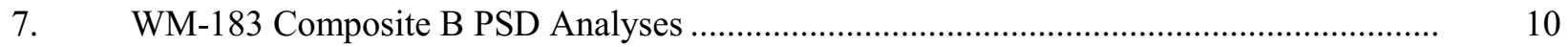

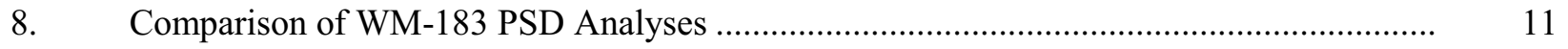

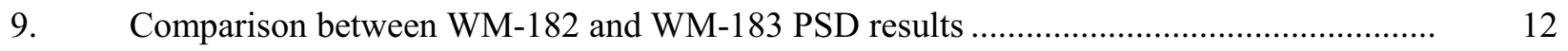

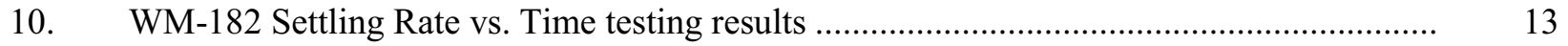

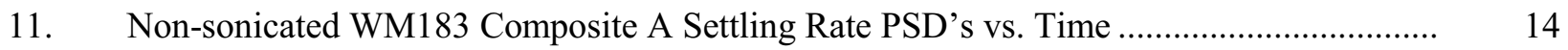

12. WM-183 Composite A non-sonicated settling rate testing. ............................................ 15

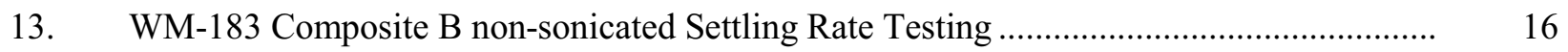

A-3a. WM-183 Composite A settling rate testing photographs ............................................... 32

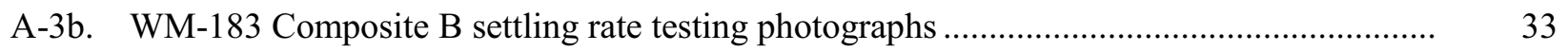

\section{TABLES}

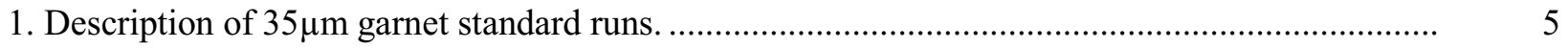

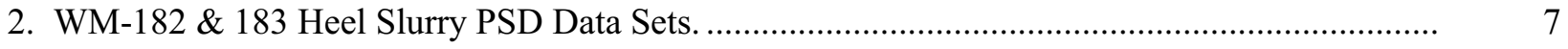




\section{Tank Farm WM-182 and WM-183 Heel Slurry Samples PSD Results}

\section{INTRODUCTION AND BACKGROUND}

As part of a sampling and physical characterization task, a laser diffraction (classical light scattering) particle size analyzer was used to determine particle size distribution characteristics of a radioactive slurry. Spent nuclear fuel was previously reprocessed at the INTEC (formerly recognized as the Idaho Chemical Processing Plant) utilizing liquid-liquid extraction processes. The acidic, radioactive aqueous streams from these processes were transferred to 300,000 gallon stainless steel storage vessels in the INTEC Tank Farm area, where each vessel sits below grade, and is totally enclosed in a concrete vault. This radioactive liquid was subsequently transferred to a solidification process. Due to the liquid transfer piping configuration in the tank farm vessels, 100 percent of this liquid could not be retrieved. Consequently, a liquid "heel" remains at the bottom of the "emptied" vessel. It is the particle size distribution (PSD) analysis of the solids in this radioactive heel slurry that is addressed in this report.

Heel slurry samples from INTEC tank farm vessels WM-182 and WM-183 were taken utilizing the Light Duty Utility Arm (LDUA) from October 1999 to January 2000. A description of this LDUA technology is presented by Patterson [1]. Tank farm samples were transferred to the INTEC Remote Analytical Laboratory (RAL) facility. Particle size analyses on these samples were performed in the RAL using a Horiba PSD analyzer which was modified for remote application. This technology provides rapid and simple PSD analysis, especially down in the fine and microscopic particle size regime. Particle size analysis of these radioactive slurries down in this smaller range was previously not achievable - making this technology far superior than the traditional particle sizing methods used before. Remote deployment and utilization of this technology is in an exploratory stage. In light of development of closure strategies for the INTEC tank farm within the auspices of the Draft High-Level Waste and Facility Disposition Environmental Impact Statement, these PSD analyses, in conjunction with other characterization analyses, are tremendously useful fundamental engineering data.

\section{PSD ANALYSIS EQUIPMENT \& METHODS}

\subsection{Remote PSD Analyzer and Equipment}

Particle size distribution analysis of INTEC Tank Farm WM-182 and -183 heel slurry samples were performed using the modified Horiba Instruments Inc. Model LA-300 laser scattering particle size distribution analyzer; it has a 0.1 to 600 micron $(\mu \mathrm{m})$ measurement range and weighs $55 \mathrm{lbs}$. This instrument was chosen for this PSD analysis task primarily because it satisfied a 12 inch wide RAL transfer tunnel dimension restriction - and because of its smaller "footprint". A description of this analyzer and some of the theory of this technology are presented in References 2 and 3.

The Horiba software generates the PSD as a discrete frequency distribution of particle volume percent versus the particle diameter. A frequency distribution is typically presented as a histogram. A differential frequency distribution, or in this case, a differential volume percent distribution curve can be approximated by drawing a smooth curve through the histogram[4]. In this way, the particle size distribution is easily grasped because the subtleties of the distribution are revealed (particularly when plotted on a logarithmic scale). Differential curves are better than histograms when comparing overlayed PSD's. The Horiba's 0.1 to $600 \mu \mathrm{m}$ particle size range is resolved into 64 logarithmically spaced channels. The channel volume percent value is matched with the channel center value. The particle size distribution and PSD statistics are calculated using the $64\{$ Vol. \% , particle size $\}$ data pairs. For greater 
flexibility of data handling and presentation purposes just described, all PSD analyses presented here were regenerated in EXCEL software by exporting the Horiba PSD data and generating a graph from this data. A cumulative volume percent plot can be obtained by summing the data (which necessarily sums to $100 \%$ ). An overlay of cumulative distributions is not as visually informative as an overlay of the differential distributions. Typically, solids-liquid-separation technologies use the cumulative $50 \%$ particle size as the "nominal" particle size that is retained by the equipment; this is reported as the median in the data presented here.

A Jencons PowerPette battery-powered pipettor with a disposable $2.2 \mathrm{ml}$ plastic pipette tip was used to draw small aliquots for remote PSD analyses of the tank farm slurry samples. A small aliquot is added to the Horiba sample dispersion/circulation tank. The circulating particles from this aliquot diffract/scatter some of the laser light. Typically a minimum of $5 \%$ obscuration (or $95 \%$ transmittance) of the light is required to ensure a diffraction/scatter pattern adequate for the analyzer to deconvolute the particle size distribution. Aliquots of slurry are added to the analyzer until satisfactory obscuration is achieved. Further technique details are presented in Reference 3. Prior to using the plastic pipettes in the RAL cell, the slurry-draw end of the tip was reamed out to approximately $2100 \mu \mathrm{m}$. This opening is over three times the analyzer's $600 \mu \mathrm{m}$ upper detection limit but it is not so large that it allows the aliquot to dribble out.

\subsection{Analyzer Performance Check with Standards}

Standards were used to demonstrate and verify analyzer performance during testing. These accuracy checks were performed during the metamorphosis of the modified analyzer and then during this tank farm slurry sample PSD analyses testing. Several Coulter Corporation particle size standards were used. A $35 \mu \mathrm{m}$ nominal mode garnet LS Control G35D (Lot: 1014) was used predominately throughout this testing. A mixture of $2.1 \mu \mathrm{m}$ modal polyvinyl toluene (Lot: 1630) and $20.8 \mu \mathrm{m}$ modal polystyrene microspheres (Lot: 5740), and a $0.3 \mu \mathrm{m}$ latex LS Size Control L300 (Lot: 1019) were also used. Typically the standards material was added directly to the Horiba sample dispersion/circulation tank.

\subsection{Tank Farm Samples, PSD Analysis Method and Testing}

The PSD analysis method sequence basically involved: 1) adding fresh water dispersant to the analyzer dispersion/circulation tank, 2) circulating and debubbling the water dispersant, 3) align the laser, 4) baseline (or "blank") the instrument, 5) add sample aliquots and perform analysis; the details of this procedure are presented in References 3 and 5.

Actual sample locations (mapped by sample number... as was done for WM-188 [1]) within the WM-182 and -183 tanks was not available at time of publication. Supposedly, sample locations are in a similar sampling pattern as to those of WM-188 [6]. A concern was raised because it was felt that a representative cross-section of the tank solids is not achievable with this sampling pattern. However, the limitations of the LDUA reach were reiterated.

In any event, there were two types of PSD analyses testing performed using the WM-182 and -183 samples: 1) typical PSD analysis testing, and 2) settling rate testing. As described earlier, small aliquots were drawn from a sample dispersion until the amount of particles circulating in the analyzer were satisfactory for an analysis by the instrument. For at least one of the tests performed, aliquots were drawn directly from an intact, non-fractionated sample dispersion. However, most testing was performed with a redispersion of solids from the original tank farm sample. And for some tests, the solids were from a composite of fractionated solids from several tank farm samples. 
For the typical PSD analyses, aliquots were drawn from a just-agitated slurry sample dispersion container using the pipettor and an analysis run performed. Duplicate analysis runs for this sample dispersion were performed until reasonable repeatability and "trending" were observed between the runs. This qualitative judgement was made by noting the mode location and, the breadth and shape of the differential PSD profile curve from one run to the next. Often this may take only three runs to clearly establish the "true" sample PSD profile. The Horiba aliquot dispersion/circulation tank has a $13 \mathrm{~W}$, $28 \mathrm{kHz}$ ultrasonic element. It was observed that use of this high frequency ultrasonic element affected the PSD profile; this is discussed in the Results section.

For the settling rate testing, a quantity of solids was fully agitated/dispersed in a $250 \mathrm{ml}$ glass graduated cylinder and then allowed to settle. At a predetermined settling time, aliquots were drawn from a point about 4" below the surface and the analysis quickly executed. This testing basically looked at the transient particle size distribution for the solids in the "clarifying" layer above the settled solids layer (at the bottom). As testing proceeded, it became apparent that the ultrasonic element should not be used for the settling rate testing PSD analyses; this is also discussed later. In this format, a settling rate PSD versus time plot was generated. Due to limitations, duplicate settling rate test runs were not performed. 


\section{RESULTS AND DISCUSSION}

\subsection{Particle Size Standards Results}

Results for the $35 \mu \mathrm{m}$ nominal mode garnet standard analyses performed over the duration of this testing are presented in Figure 1.

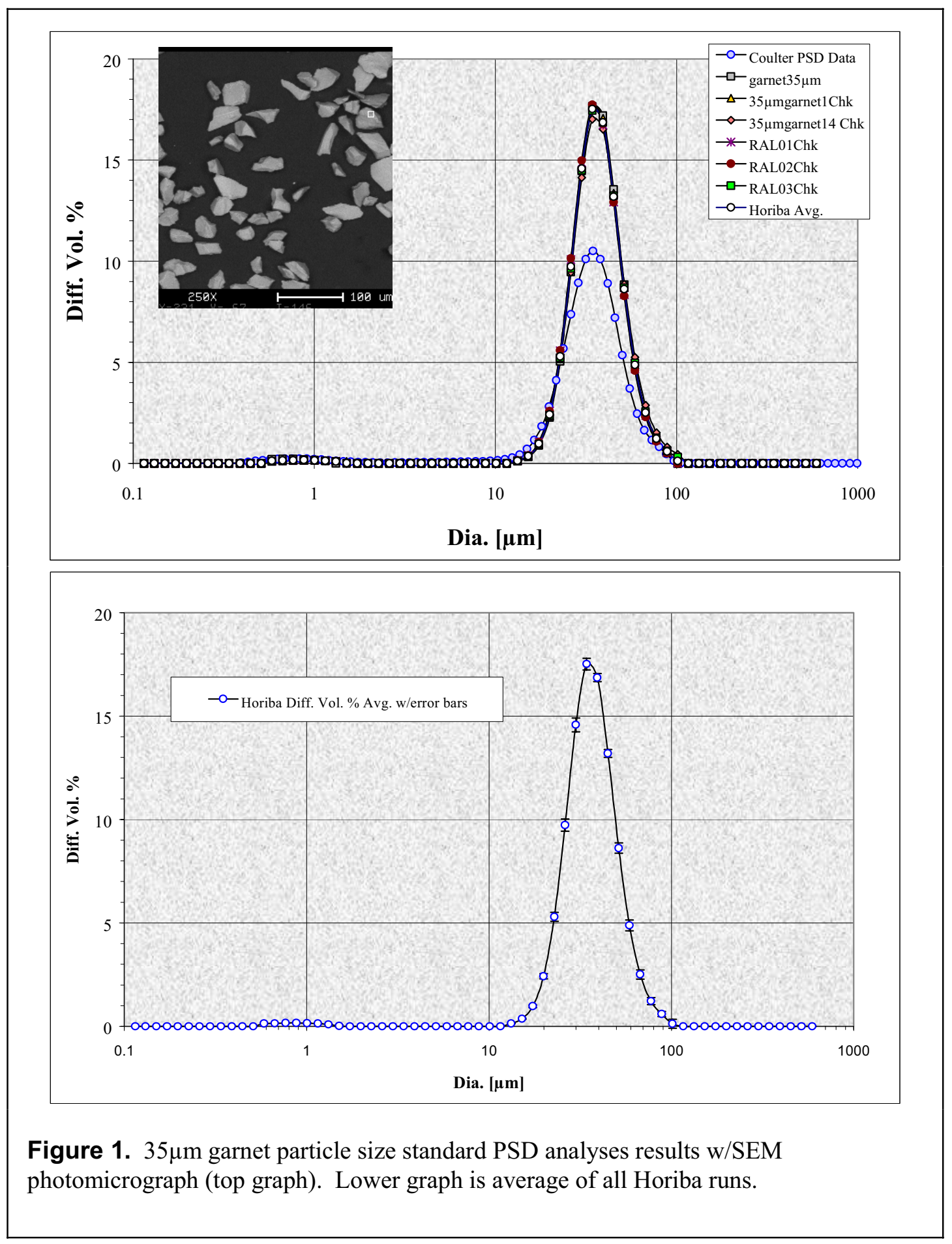


Run descriptions for these $35 \mu \mathrm{m}$ garnet runs are given in Table 1 .

Table 1. Description of $35 \mu \mathrm{m}$ garnet standard runs.

\begin{tabular}{|c|c|c|}
\hline Filename & Description & Date \\
\hline garnet $35 \mu \mathrm{m}$ & performed upon receipt and setup of unit at INTEC. & $26 \mathrm{Jul} 99$ \\
\hline $35 \mu$ mgarnet $1 \mathrm{Chk}$ & after completion of major modifications to unit & 2 Nov 99 \\
\hline $35 \mu \mathrm{mgarnet} 14 \mathrm{Chk}$ & upon final assembly of unit & 29 Nov 99 \\
\hline RAL01Chk & $\begin{array}{l}\text { first analysis performed with unit in RAL cell— just prior to first "hot" } \\
\text { sample from WM-182 }\end{array}$ & 23 Dec 99 \\
\hline RAL02Chk & $\begin{array}{l}\text { check instrument performance prior to continued analyses of tank farm } \\
\text { samples }\end{array}$ & 19 Jan 00 \\
\hline RAL03Chk & $\begin{array}{l}\text { check instrument performance prior to continued analyses of tank farm } \\
\text { samples }\end{array}$ & 7 Feb 00 \\
\hline
\end{tabular}

It is clear from these results with the garnet standard that the modifications to the unit for remote application at the RAL did not affect the analyzer, and the instruments level of repeatability remained high. However, it was noted that the Horiba consistently yielded a mode down around $32 \mu \mathrm{m}$ for this standard (see Appendix A.1; analyzer PSD data in the EXCEL spreadsheet format and statistics for WM-182 and the rest of the standards are in Appendix A.1). Analysis of this standard performed with a Coulter LS230 laser diffraction particle size analyzer is also shown in Figure 1 (top graph); the Coulter consistently yielded the mode at around $35 \mu \mathrm{m}$. This raised a concern regarding the calibration of the Horiba.

As a follow-on to this concern, a mixture of 2 and $21 \mu \mathrm{m}$ standards was analyzed in the Horiba before deployment of the analyzer in the RAL; the results for this are presented in Figure 2.

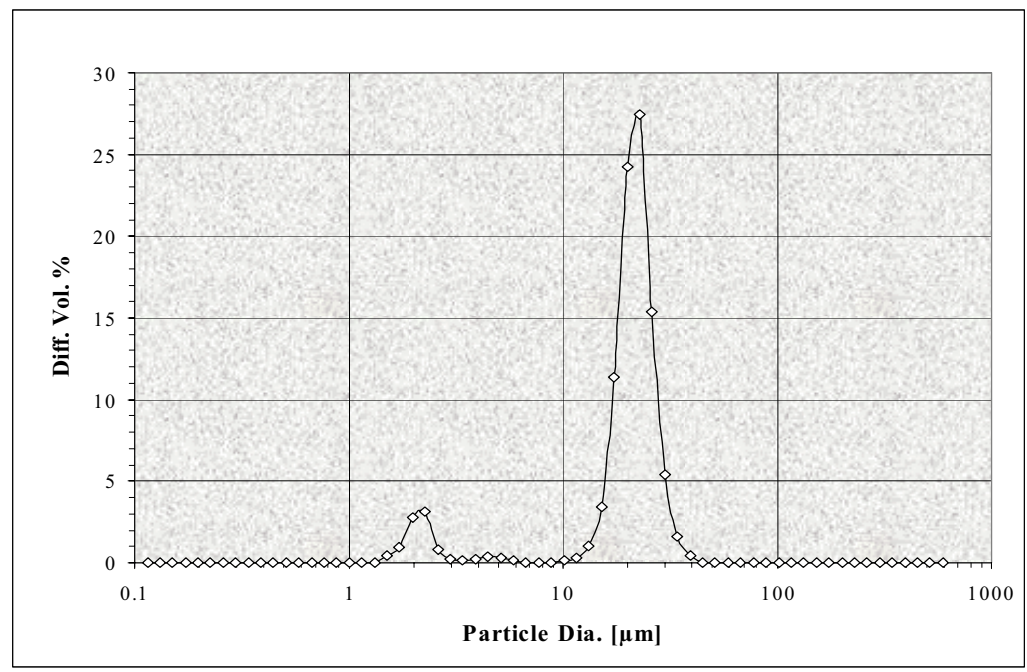

Figure 2. Mixture of Coulter standards; 2.1 and $20.8 \mu \mathrm{m}$ microspheres; 2 Nov 99.
As can be seen, these results were quite satisfactory (see data table in Appendix A.1). It was decided at this juncture that the Horiba was operating within an acceptable reproducibility range, and that there was not a calibration discrepancy of such magnitude so as to preclude deployment of the Horiba in the RAL.

In the course of the tank farm testing, a curious observation was that the PSD's showed zero volume percent of material less than about $0.5 \mu \mathrm{m}$. It was expected that some of the samples would show a non-zero volume percent down to the 
instrument's $0.1 \mu \mathrm{m}$ lower limit. An analysis of the Coulter $0.3 \mu \mathrm{m}$ standard was performed to demonstrate that the instrument was detecting particles less than $0.5 \mu \mathrm{m}$. The result for this test is presented in Figure 3. This result indicated that the Horiba was detecting particles less than $0.5 \mu \mathrm{m}$.

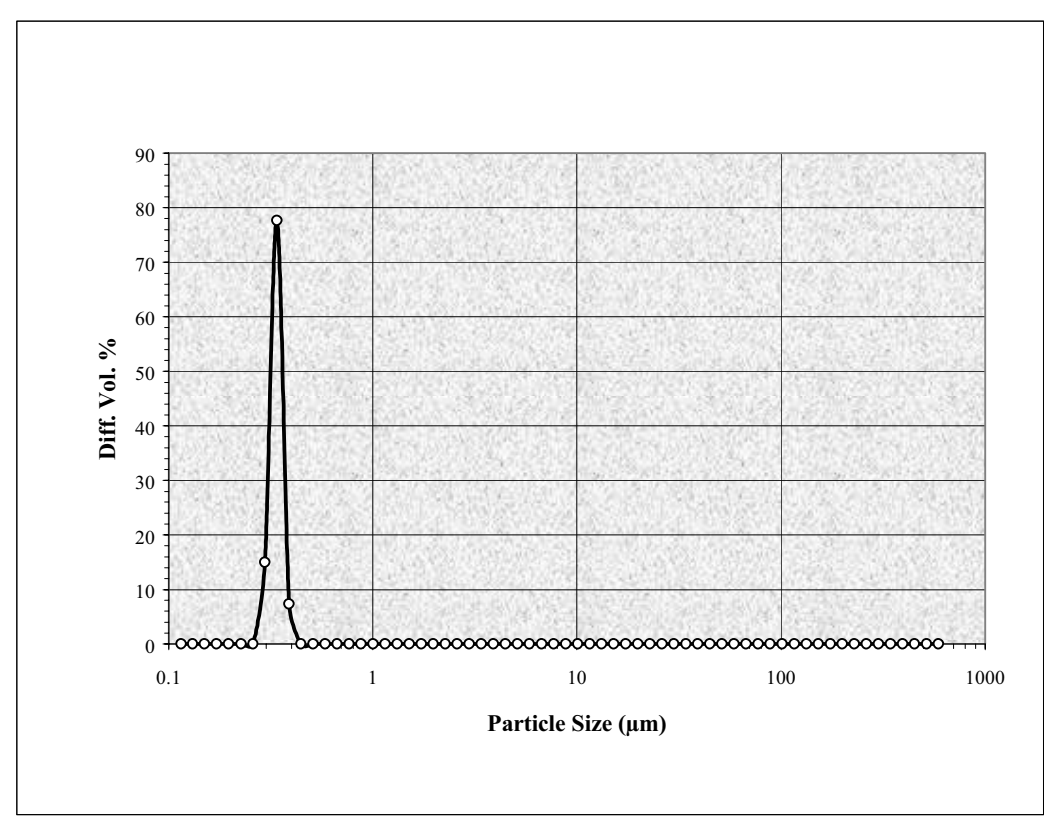

Figure 3. Coulter $0.3 \mu \mathrm{m}$ latex size standard; 11 Apr 2000.

The overall results for the standards testing were satisfactory and demonstrated that the Horiba was performing with excellent repeatability and acceptable accuracy during the actual tank farm PSD testing.

In order to decrease the abrasive wear on the Horiba's glass sample cell, a move to a glass bead standard instead of the garnet is recommended. Continued use of the engineered monosize spheres is satisfactory for checking analyzer performance at specific sizes. Standard Reference Material such as glass bead standards are available

from the National Institute of Standards and Technology (NIST) for checking performance over a range of sizes; a range of from 34-to- $120 \mu \mathrm{m}$ is recommended.

\subsection{Tank Farm Heel Slurry Sample PSD Results}

For the tank farm heel slurry sample typical PSD analysis testing, and the settling rate testing, there were seven sets of PSD data. These data sets are shown in Table 2. 
Table 2. WM-182 \& 183 Heel Slurry PSD Data Sets.

\begin{tabular}{|c|c|c|c|}
\hline & LDUA Sample & Description & Date \\
\hline \multirow{4}{*}{ 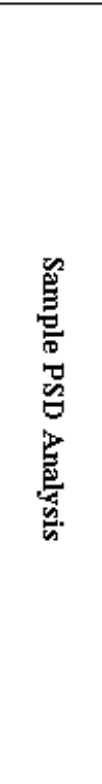 } & WM-182 \#4 & $\begin{array}{l}\text { Non-fractionated slurry from sample LN } 9911082 \\
\text { Aliquot dispersion in Horiba was not sonicated. } \\
\text { Aliquot dispersion in Horiba was sonicated. }\end{array}$ & $\begin{array}{l}23 \operatorname{Dec} 99 \\
1 \overline{1} \overline{A p r} \overline{0} \overline{0}\end{array}$ \\
\hline & WM-183\#3 & $\begin{array}{l}\text { Solids redispersed from suspended solids fraction of sample LN } 0001125 \text {. } \\
\text { Dispersion in Horiba was not sonicated } \\
\text { Dispersion in Horiba was sonicated. }\end{array}$ & $19 \mathrm{Jan} 00$ \\
\hline & $\begin{array}{l}\text { WM-183 } \\
\text { Composite of } \\
\text { Sample \#'s } 1,2 \text {, } \\
\text { and } 3 \\
\text { (Composite } A \text { ) }\end{array}$ & $\begin{array}{l}\text { Solids composited and redispersed from settled solids fraction from sample } \\
\text { LN's } 0001056,0001123 \text { and } 0001125 \text {. In this report, this composite is } \\
\text { designated as Composite A. } \\
\text { Dispersion in Horiba was not sonicated. }\end{array}$ & $19 \operatorname{Jan} 00$ \\
\hline & $\begin{array}{l}\text { WM-183 } \\
\text { Composite of } \\
\text { Sample \#'s } 4,5,6 \text {, } \\
\text { and } 7 \\
\text { (Composite B) }\end{array}$ & $\begin{array}{l}\text { Similarly, solids composited and redispersed from settled solids fraction } \\
\text { from sample LN's } 0001175,0001176,0001191 \text { and } 0001192 \text {; this } \\
\text { composite is designated as Composite B. } \\
\text { Dispersion in Horiba was not sonicated. } \\
\text { Dispersion in Horiba was sonicated. }\end{array}$ & 7 Feb 00 \\
\hline \multirow{3}{*}{ 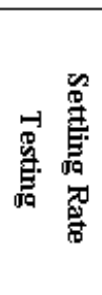 } & WM-182\#4 & $\begin{array}{l}\text { - Non-segregated slurry from sample LN } 9911082 . \\
\text { - Aliquot dispersion in Horiba was sonicated. }\end{array}$ & $8 \mathrm{Feb} 00$ \\
\hline & $\begin{array}{l}\text { WM-183 } \\
\text { Composite A }\end{array}$ & $\begin{array}{l}\text { - Testing performed with Composite A material (as described above). } \\
\text { - Aliquot dispersion in Horiba was not sonicated. }\end{array}$ & 8 Mar 00 \\
\hline & $\begin{array}{l}\text { WM-183 } \\
\text { Composite B }\end{array}$ & $\begin{array}{l}\text { - Testing performed with Composite B material (as described above). } \\
\text { - Aliquot dispersion in Horiba was not sonicated. }\end{array}$ & 8 Mar 00 \\
\hline
\end{tabular}




\subsubsection{WM-182\#4 PSD Analysis}

The WM-182 Sample \#4 PSD analyses were the first performed on actual $\gamma$ radiation material at the RAL with the newly installed Horiba. For the first five runs performed, the ultrasonic element (described previously in Section 2.3) which sonicates the liquid in the analyzer's aliquot dispersion/circulation tank, was not used. Sonication was used later and was found to significantly affect the PSD profile. The average PSD's for non-sonicated versus sonicated runs are presented in Figure 4. Initially, it was believed that sonication dispersed agglomerated particles and yielded the fundamental particle sizes in the sample. However at a later date, SEM photomicrographs of WM-182 solids (see Figure 4 insert) became available for inspection. It was then believed that the larger particles were not comprised of smaller, agglomerated particles. Moreover, the results for the unsonicated case represented the "as is" particle size distribution for the tank slurry sample (along the lines as what is seen in the SEM insert) - and the possibility that sonication is actually "breaking-up" larger particles was raised. This finding represents useful information regarding ultimate tank heel waste processing. Further investigation of sonication effects is needed and is recommended.

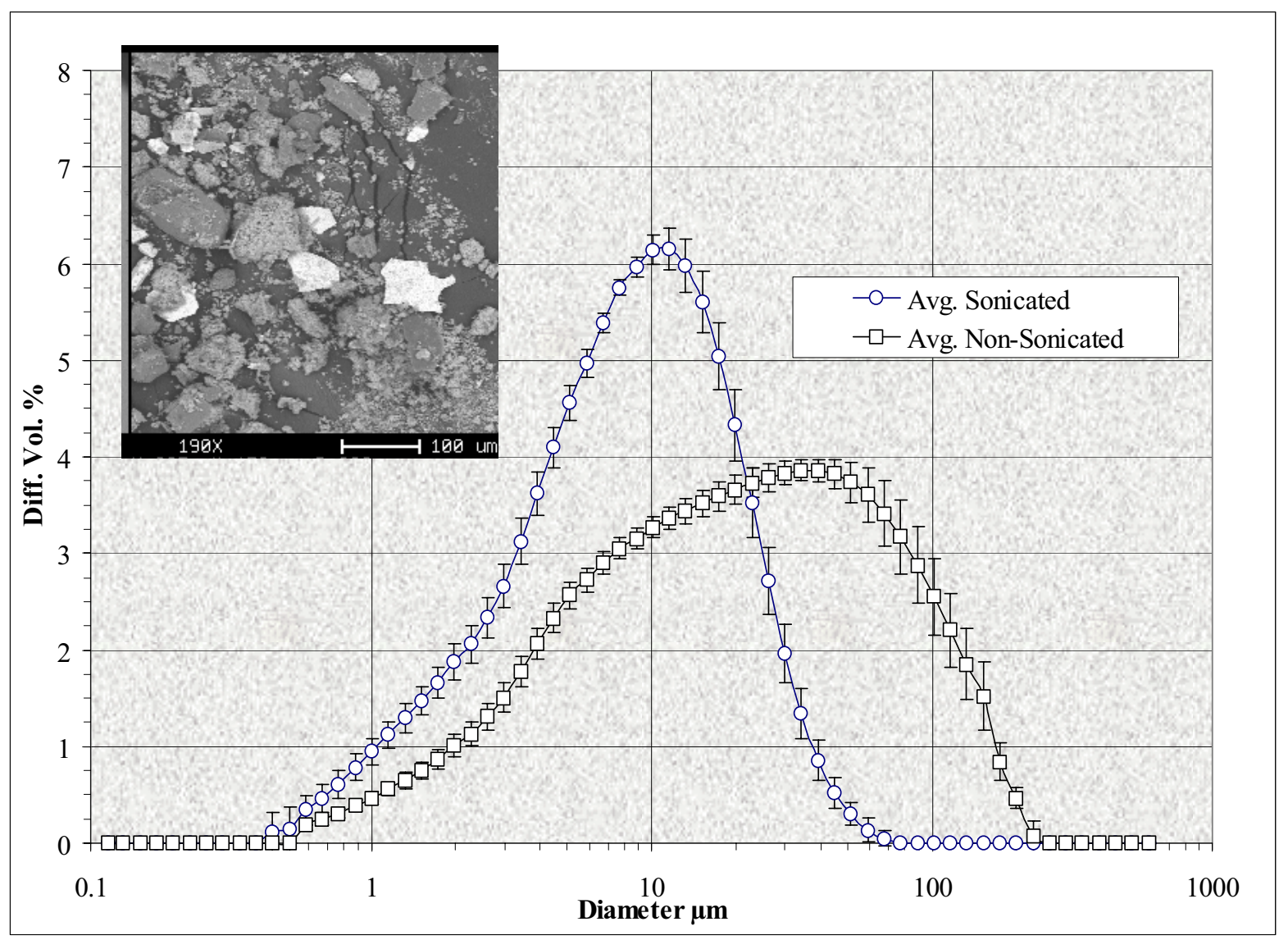

Figure 4. WM-182 \#4 PSD Analyses; avg. PSD for non sonicated vs. sonicated samples. SEM photomicrograph insert of dried and mounted WM-182 slurry solids. 
The particle sizes seen in the SEM photo compares with the non-sonicated PSD profile sizes in that there are particles on-the-order of $100 \mu \mathrm{m}$, but none are seen of the size greater than $300 \mu \mathrm{m}$ (where the profile "zeroes-out" on the "big" end) — and the broad mode around $40 \mu \mathrm{m}$ can be easily supported. Obviously it can not be assumed that this photo represents the particle distribution that was "seen" by the Horiba (let alone, that of the slurry sample). Analyzer PSD data in the spreadsheet format for this WM-182\#4 sample are provided in Appendix A.2 (as are all the remaining tank farm sample PSD data).

\subsubsection{WM-183\#3 PSD Analysis}

PSD analyses were performed with solids which were redispersed from the original suspended solids fraction of WM-183 Sample \#3 (Log Number 0001125). The suspended solids fraction is decanted/separated off the top of the settled solids fraction of the sample. The effect of sonication was noted for these results also. The average PSD's for non-sonicated versus sonicated runs are presented in Figure 5. Unlike the WM-182 results, an $\sim 15 \mu \mathrm{m}$ mode shows up in both the unsonicated and the sonicated profile - albeit much less dominant in the non-sonicated case. Because this material was from the suspended solids fraction, the significant amount of larger size particles was not expected to be seen; further discussion of this is taken up later.

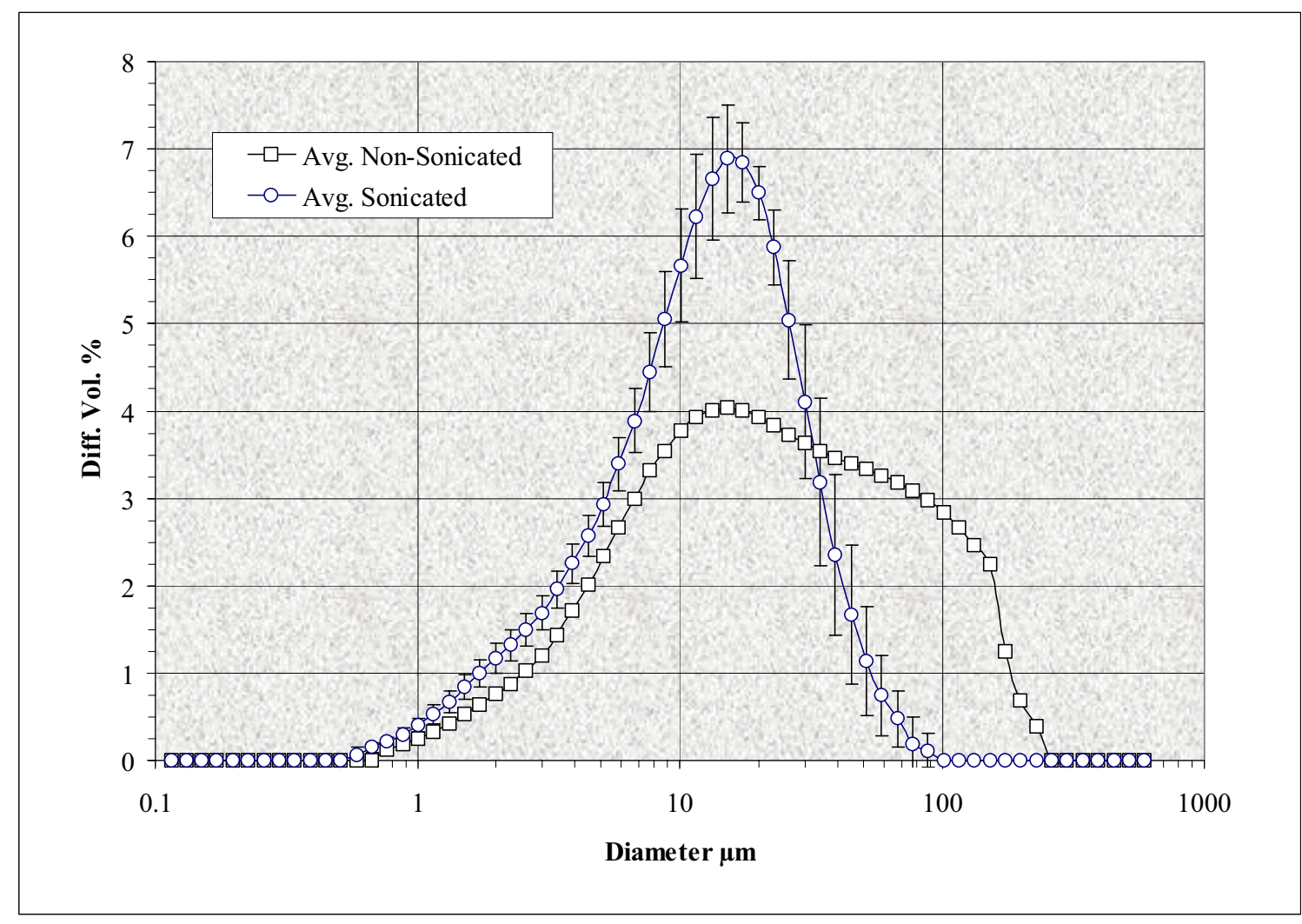

Figure 5. WM183\#3 PSD Analyses; avg. PSD for non-sonicated vs. sonicated samples. 


\subsubsection{WM-183 Composite A PSD Analysis}

PSD analyses were performed with solids which were redispersed from a composite of the original settled solids fraction of WM183 Sample \#'s 1, 2 and 3. The average PSD's for unsonicated versus sonicated runs are presented in Figure 6. Sonication had some effect on this sample. The unsonicated result shows larger particles out to $\sim 200 \mu \mathrm{m}$; however, the mode at $\sim 10 \mu \mathrm{m}$ is dominant, just like in the sonicated case. Because this material was from the settled solids fraction, a significant amount of larger size particles was expected to be seen; Again, further discussion to this is given later.

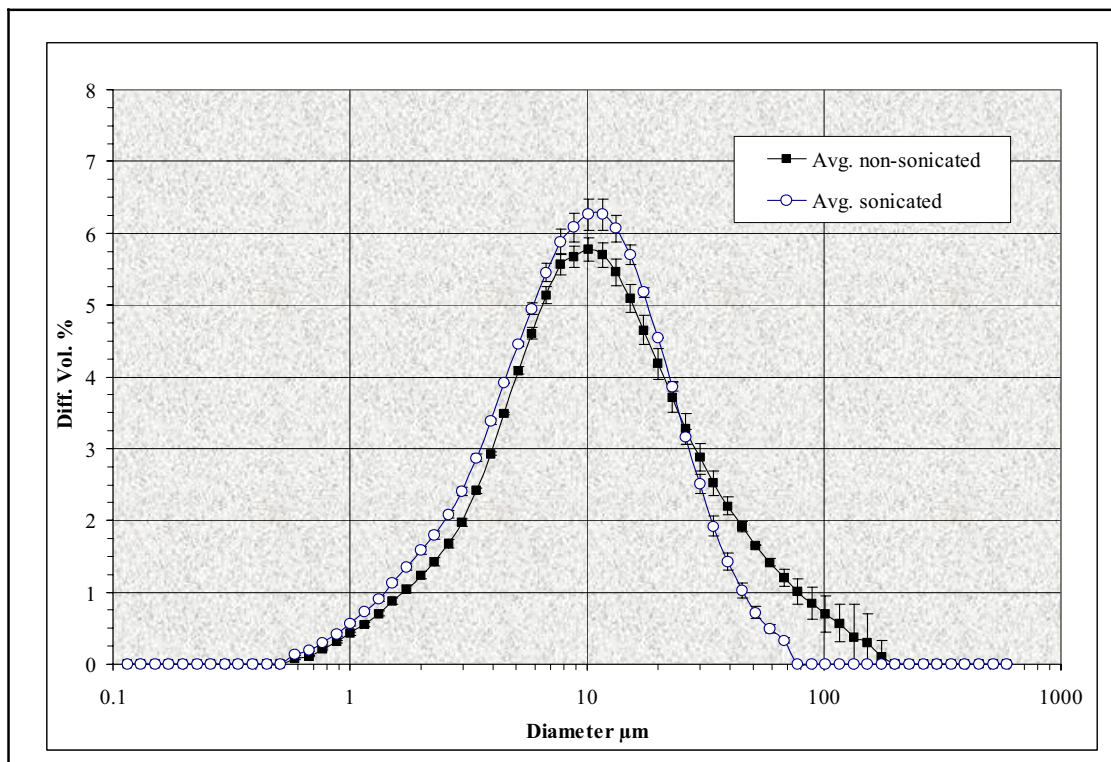

Figure 6. WM-183 Composite A PSD Analyses; avg. PSDs for non-sonicated vs. sonicated runs.

\subsubsection{WM-183 Composite B PSD Analysis}

Similarly, PSD analyses were performed with solids which were redispersed from a composite of the original settled solids fraction of WM183

Sample \#'s 4, 5, 6 and 7. The average PSD's for unsonicated versus sonicated runs are presented in Figure 7 . The effect of sonication was similar to that noted for WM-183\#3. This kind of distribution is what was expected for the settled fraction.

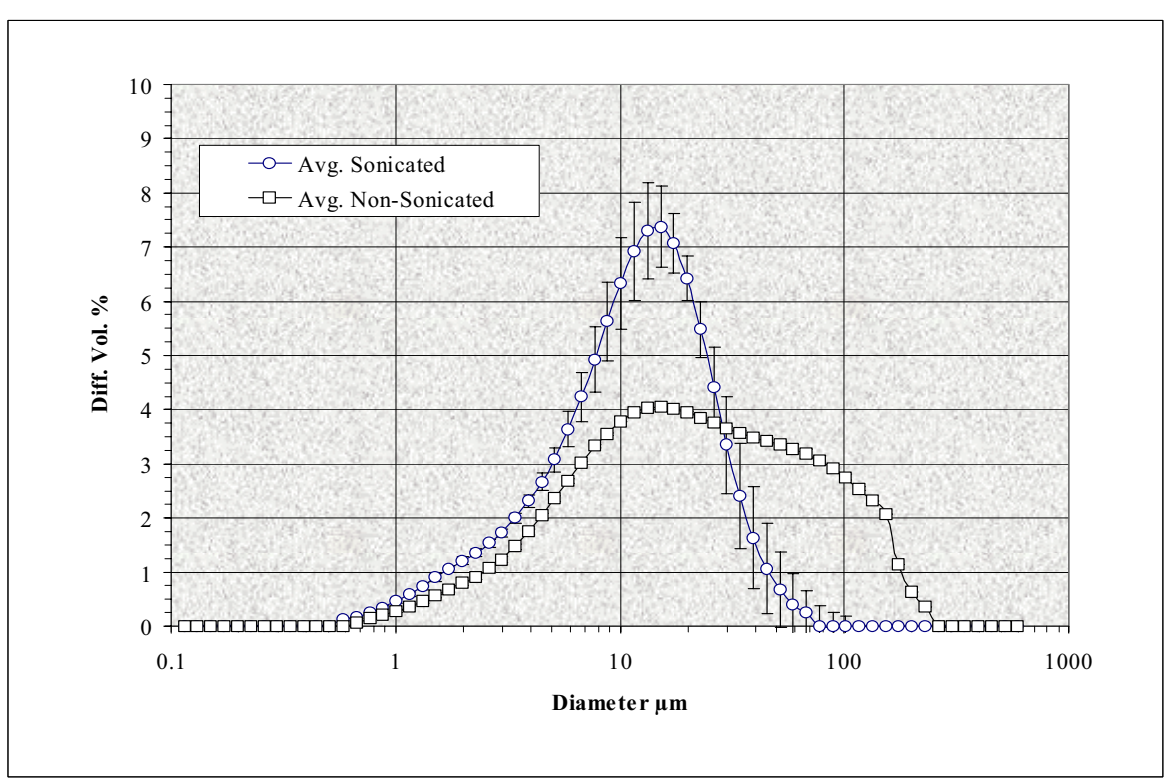

Figure 7. WM-183 Composite B PSD Analyses; avg. PSDs for non-sonicated vs. sonicated runs. 


\subsubsection{Comparison of WM-183 PSD Analyses}

A comparison of the three WM-183 sample PSD analyses is presented in Figure 8 (unsonicated and sonicated results). Per the previous deferring discussions, only Composite B results seem reasonable for settled solids fraction material. WM183\#3 and Composite A results appear to be reversed for their respective material as discussed earlier. However, concrete evidence to "correct" this anomaly has not been uncovered - the results stand as presented.
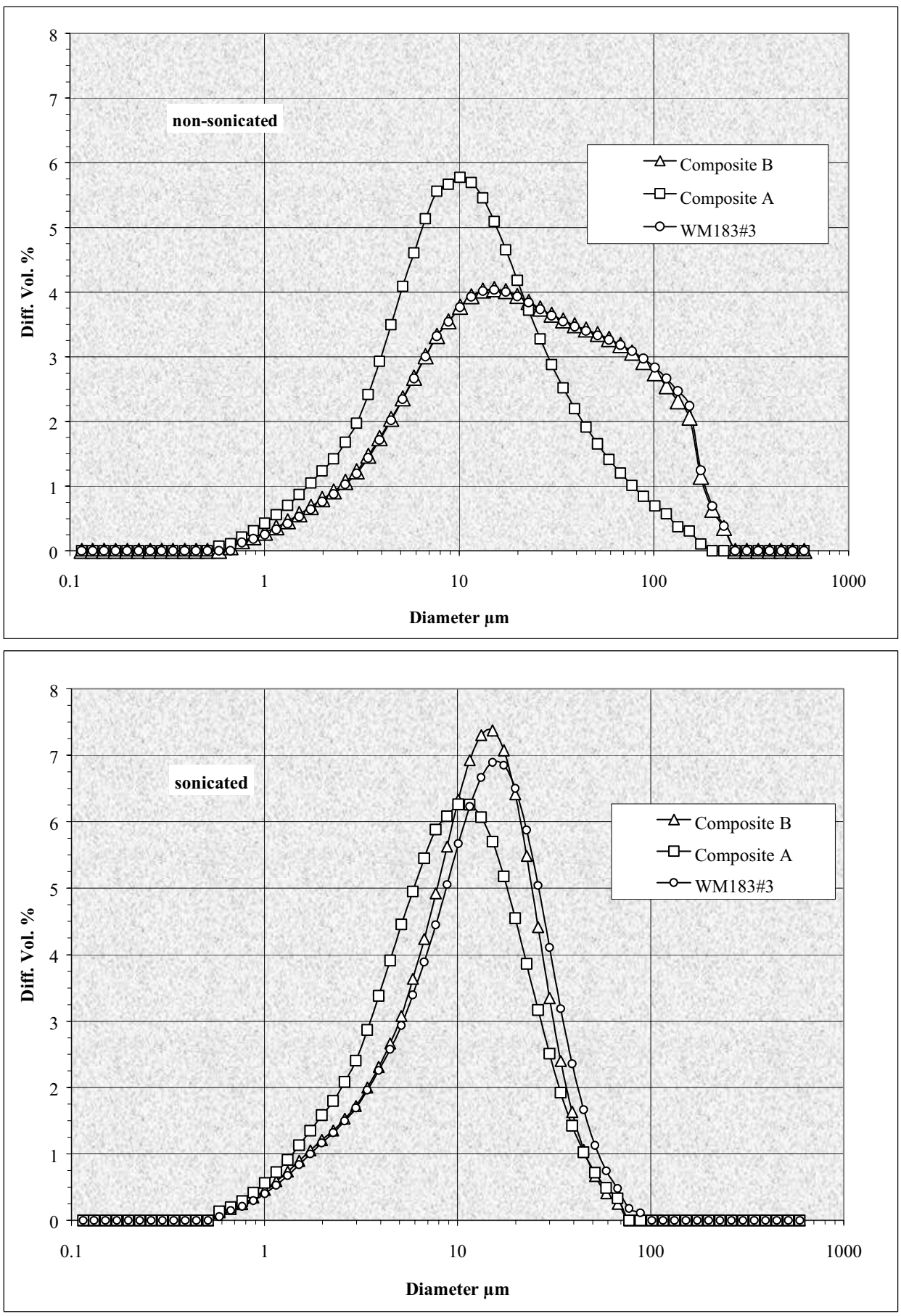

Figure 8. Comparison of WM-183 PSD analyses; avg. unsonicated (top) vs. sonicated (bottom) PSD results. 


\subsubsection{Comparison Between WM-182 and WM-183 PSD Analyses}

On one hand, the assumption that the PSD results for WM-182 and WM-183 represent the entire particle size distribution for their respective vessels is not at all statistically defensible. Conversely, considering the minute quantities used from the two separate vessels to obtain these results, the similarities between the results is noteworthy; as can be seen in Figure 9, this holds for both the non-sonicated and the sonicated case. Take note that, based on the discussion in the previous WM183 comparison section, WM183\#3 and Composite A were not included in Figure 9.

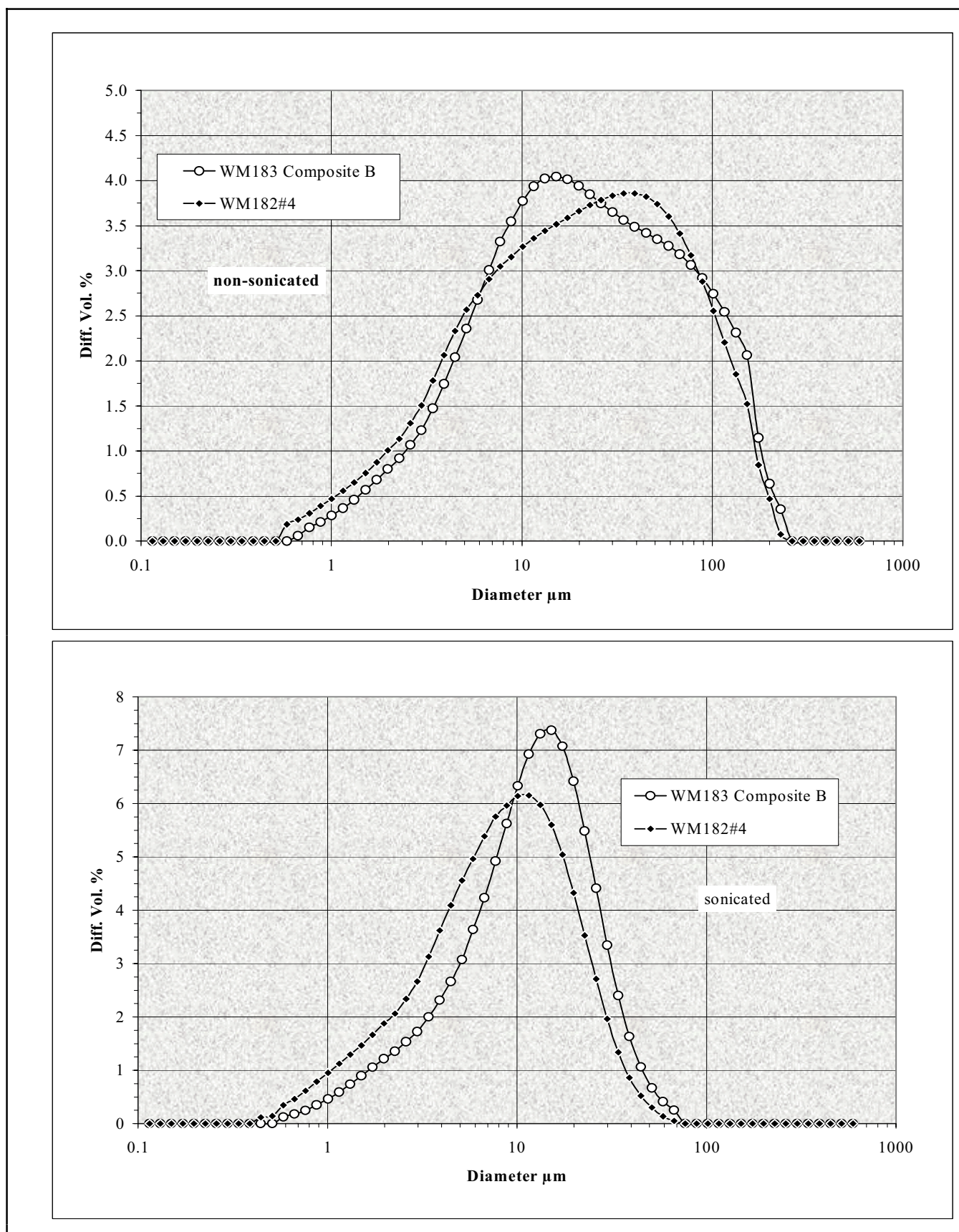

Figure 9. Comparison between WM-182 and WM-183 PSD results. 


\subsubsection{WM-182\#4 Settling Rate Testing}

Settling rate testing was performed with non-fractionated WM-182\#4 sample material. The results are presented in Figure 10. For this case, a 3-D plot was generated (the overlay plot is presented in the Appendix). When the results of this testing were reviewed in conjunction with the WM182 SEM photomicrographs (shown earlier), it became apparent that sonication was significantly affecting the PSD's and probably do not represent those of the settling solids viewed in the graduated cylinder. It was at this juncture that the decision to not use sonication for the settling rate testing PSD analyses was made.

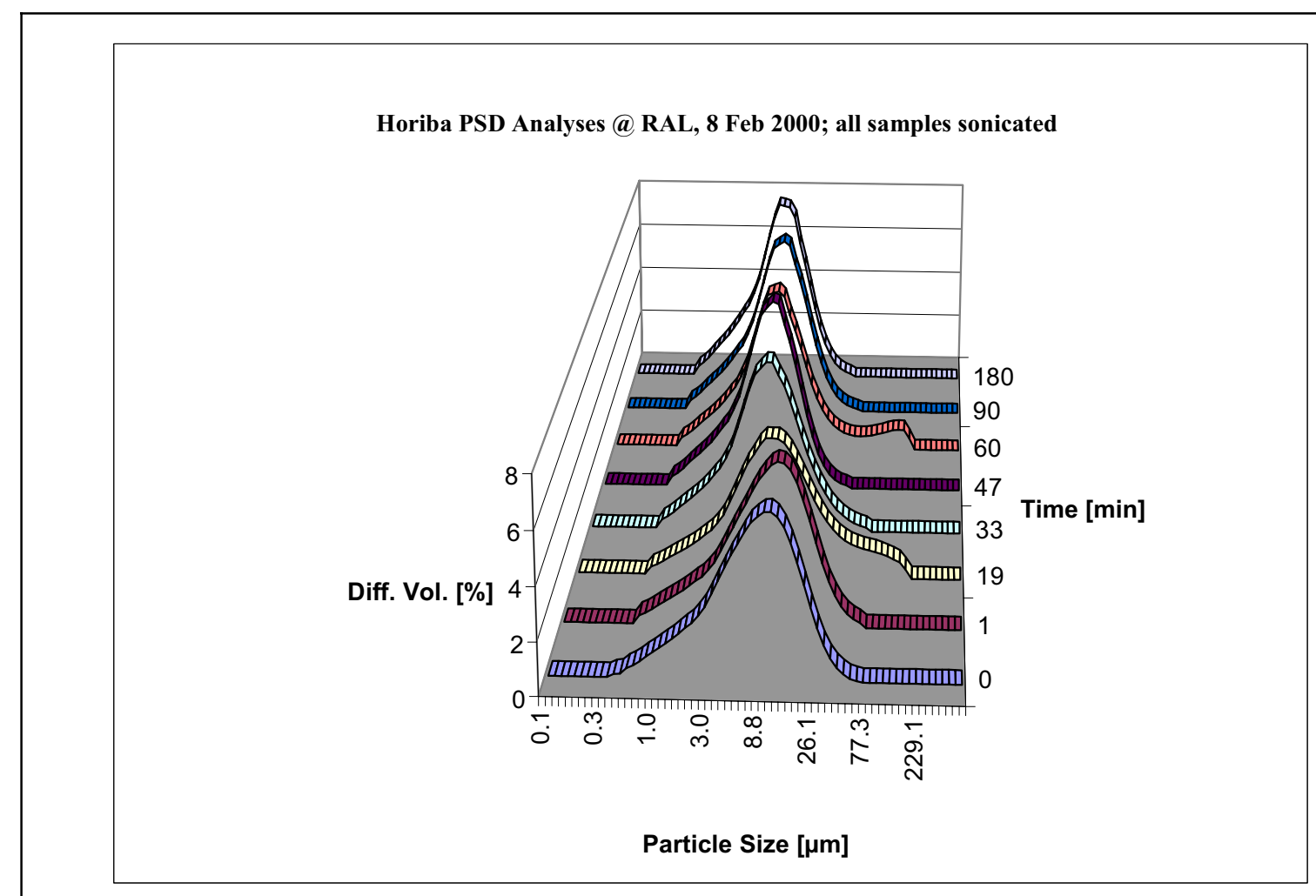

Figure 10. WM-182 Settling Rate vs. Time testing results; all samples were sonicated. 


\subsubsection{WM-183 Composite A Settling Rate Testing}

Settling rate testing was performed with WM183 Composite A material under non-sonicated conditions. These results, along with some photographs taken during this testing are presented in Figure 11. Recall from Figure 6 that this sample showedthis strong mode at $\sim 10 \mu \mathrm{m}$ even for the unsonicated

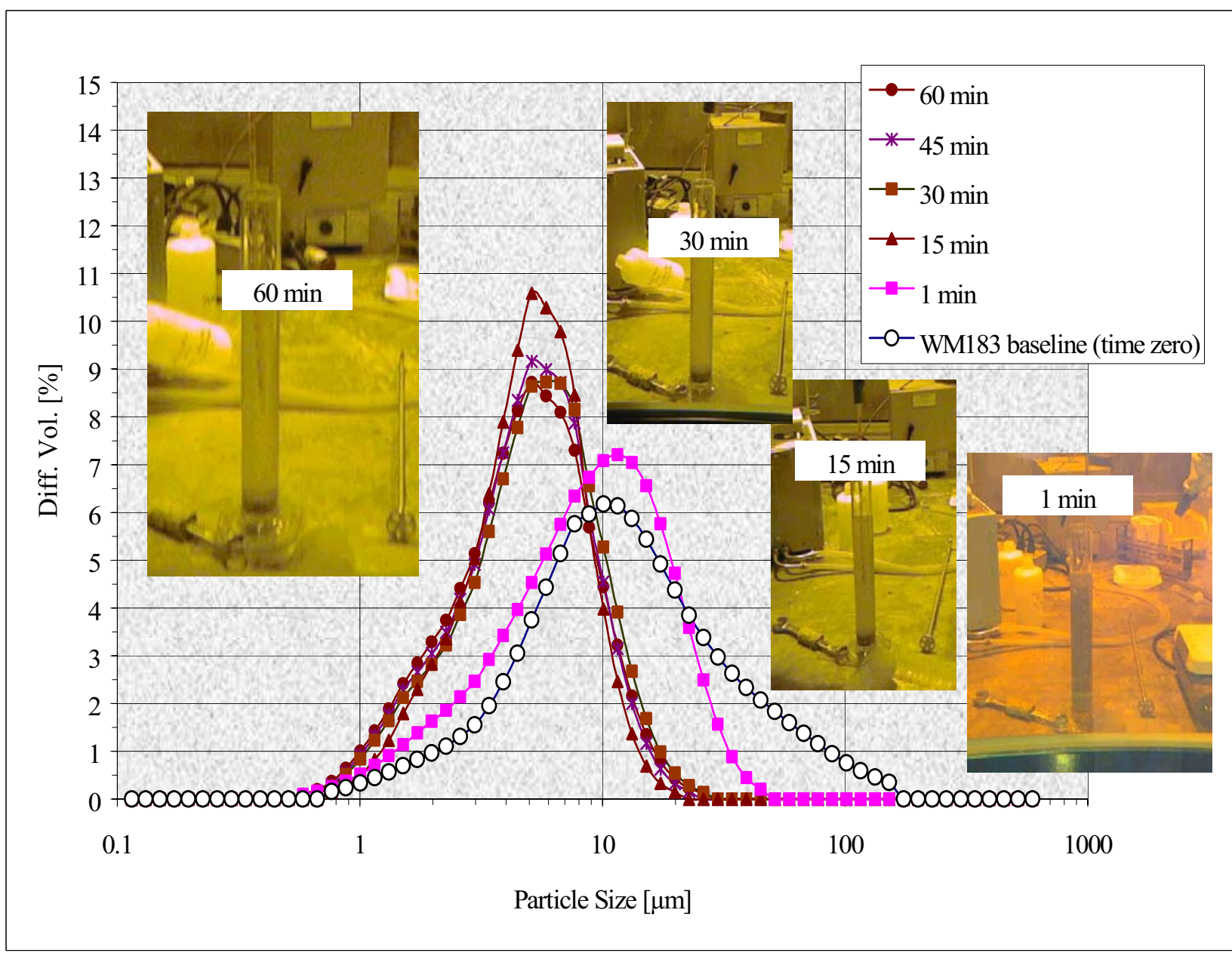

Figure 11. Non-sonicated WM183 Composite A Settling Rate PSD’s vs. Time.

case. These results show that after 15 minutes of settling time, the PSD has aligned into a distribution having a strong mode at $\sim 5 \mu \mathrm{m}$ and maximum particle size of about $30 \mu \mathrm{m}$ - this result was interestingly quite similar to the WM-182 settling test result which was performed under conditions of sonication. The one and the 30-minute photos are presented in Figure 12 for closer inspection; after 30 minutes, a distinct settled layer was observable. 


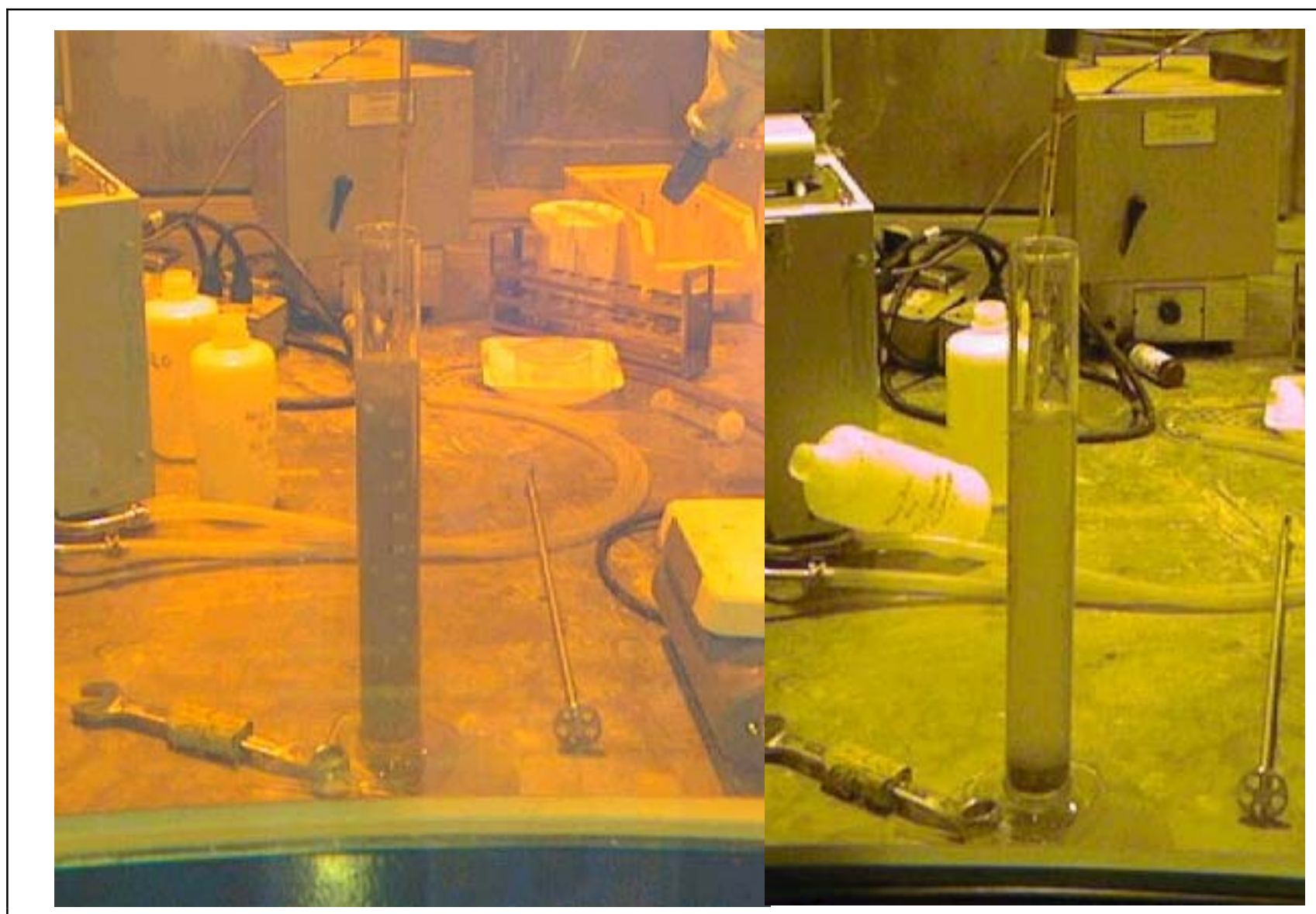

Figure 12. WM-183 Composite A non-sonicated settling rate testing; $1 \mathrm{~min}$. on left and $30 \mathrm{~min}$. on right..

A full set of the photographs taken during this Composite A settling rate testing is provided in Appendix A.3 (as is the Composite B settling rate testing set). While inspecting these, keep in mind that these photos were taken through the RAL cell shielding window, which is optically equivalent to about 3 feet thick glass; this is the reason for the poor quality for some of these photos; also take note that there is a $1.7 \times$ magnification through the window. Determination of a settling velocity and estimation of other solids physical characterization parameters (particle density, for example) were not in the scope of this work. 


\subsubsection{WM-183 Composite B Settling Rate Testing}

Settling rate testing was performed with WM183 Composite B material under non-sonicated conditions. These results, along with some of the photographs taken during this testing are presented in Figure 13. This result is what was expected for these settling rate tests.

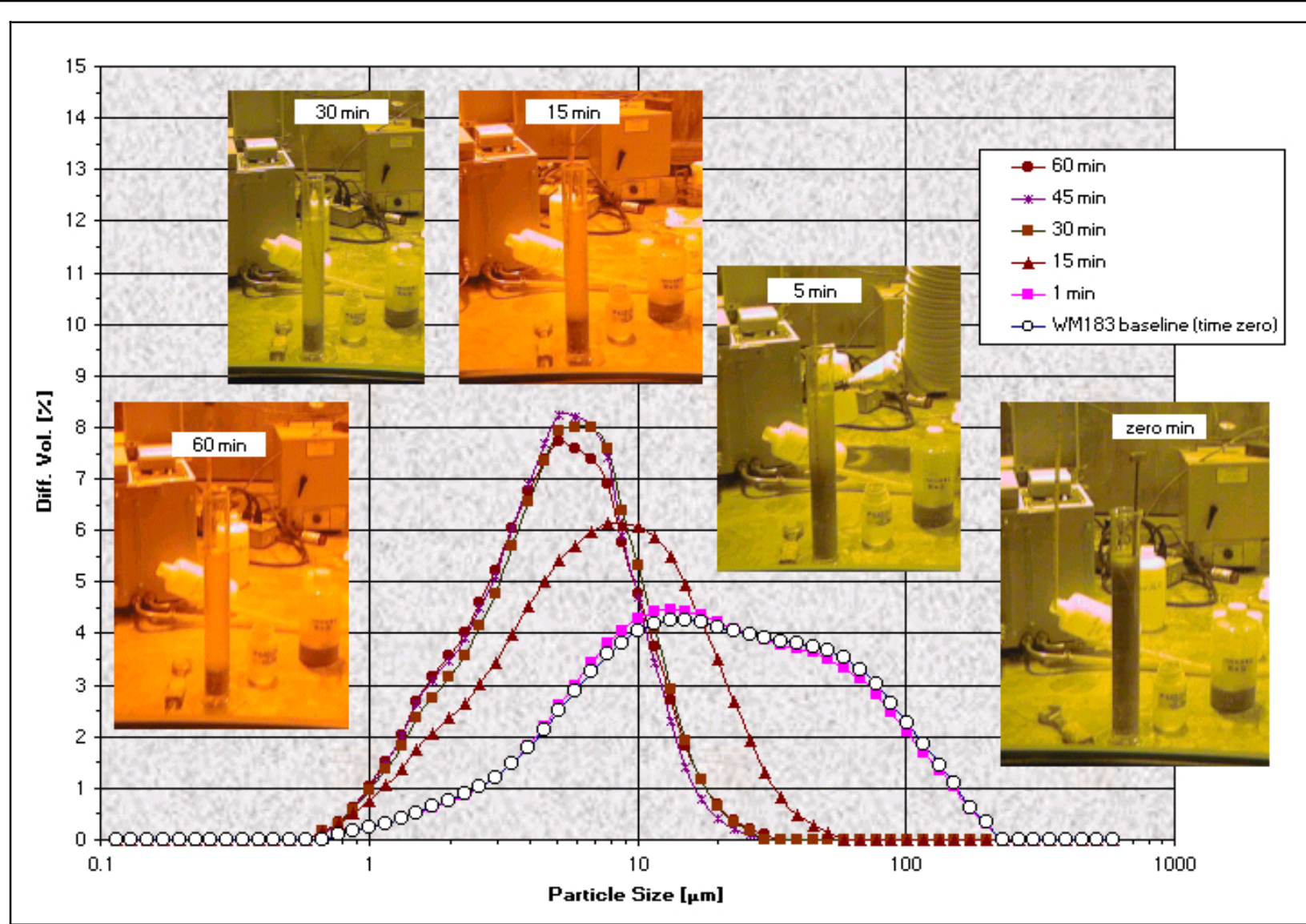

Figure 13. WM-183 Composite B non-sonicated Settling Rate Testing.

\subsubsection{Comparison Between WM-182 and WM-183 Settling Rate Testing Results}

In general, all settling rate testing results were fairly consistent in that it appears that most of the mass of solids settle to an agglomerated, yet easily redispersed layer at the bottom; and the $\sim 5 \mu \mathrm{m}$ mode and maximum $30 \mu \mathrm{m}$ size material remains dispersed/suspended in the "clear" layer (see photos) at equilibrium (that is, after about $1 / 2 \mathrm{hr}$. settling time). Also, the non-sonicated PSD results represent the particle size of the settling material and the sonicated PSD results most likely do not.

Although a non-sonicated settling rate test was not performed with WM-182\#4 sample material, the outcome would have most likely been quite similar to that observed for the WM-183 Composite B; that is, a broad distribution at one minute settling time and then alignment into the "equilibrium" PSD (described above) after 30 minutes or so. 


\subsubsection{PSD Results Closing Discussion}

Use of non-fractionated slurry is recommended; this may be a problem in that it will require more sample volume. For future settling rate testing, suspended solids concentration (loading) data should be taken for a detention test analysis as detailed in Perry's [7]. Based on the experience gained from this work, smaller interval initial settling times are needed (at least two or three before the 15 minute mark); this may require duplicate PSD settling tests because of the sampling turnaround for the Horiba. If the settling times for both the solids loading testing and the Horiba PSD testing are matched, then these data can be coupled for more robust solids/liquid separation analyses. An approximate bulk-settling rate could be determined from the photos presented in this report (this was not done in this work). As alluded to before, WM-182 and -183 solids slurries appear to have a significant "unsettleable" solids fraction with a maximum size of about $30 \mu \mathrm{m}$ and an $\sim 5 \mu \mathrm{m}$ mode.

Care must be taken in the use of this particle size data for slurry simulant formulation and in predicting slurry processing and processing equipment performance based on particle size data. Laserbased particle size differs from traditional screen size. Because most materials are not perfectly spherical, screen sizing biases toward the shortest particle dimension. The laser diffraction particle size instrument measures the particle dynamic average size between the longest and the shortest particle dimension. A traditional screen size distribution is based on mass percent; as alluded to earlier, laser size distribution is based on volume percent. Take note that in this report only laser instrument particle size is used.

Although it can be a very crucial parameter, particle shape considerations are not addressed in this report. Comparison of these tank farm data presented here against laboratory testing data obtained with classical light scattering particle sizing technology will be the ideal case, and is recommended.

An interesting and fortuitous situation occurred in that — particle sizes greater than $300 \mu \mathrm{m}$ were not observed for any of these WM-182 and -183 samples. This may have been an artifact of the LDUA sampling procedure (the $2100 \mu \mathrm{m}$ pipette tip opening should have precluded a bias problem with the Horiba aliqouting procedure). Further investigation of this was not in the scope of this work. In the future, if tank farm sample particle sizes exceed the Horiba's $600 \mu \mathrm{m}$ upper limit, the sample will require a prescreening preparation to remove the larger sizes (referred to as "scalping"). The prescreen sieve data would require "splicing" to the laser diffraction sizing data to generate a continuos distribution. 


\section{CONCLUSIONS AND RECOMMENDATIONS}

Particle size distribution analysis of the WM-182 and WM-183 tank farm heel slurry samples was performed with a modified Horiba LA 300 laser diffraction PSD analyzer. There were two types of testing performed: 1) typical PSD analysis, and 2) settling rate testing. Particle size standards were used to demonstrate and verify the performance of the analyzer during testing. The conclusions and recommendations based on the results of this work follow:

1. The overall results for the standards testing were satisfactory and demonstrated that the Horiba was performing with excellent repeatability and acceptable accuracy during the actual tank farm PSD testing.

2. Use of a NIST 34-to- $120 \mu \mathrm{m}$ glass bead Standard Reference Material is recommended over the more abrasive $35 \mu \mathrm{m}$ garnet used during his testing. This glass bead standard is for checking over a range of sizes. Continued use of engineered monosize spheres is satisfactory for checking analyzer performance at specific sizes.

3. Particle size distribution analysis showed that for both the WM-182 and WM-183 samples, the particles range approximately from a minimum of 0.5 to a maximum of $230 \mu \mathrm{m}$ - with about 90 Volume \% between approximately 2 to $133 \mu \mathrm{m}$. The WM-182 sample had a moderate mode at $32 \mu \mathrm{m}$ while the WM-183 mode was at $14 \mu \mathrm{m}$. On one hand, the assumption that the PSD results for WM-182 and WM-183 represent the entire particle size distribution for their respective vessels is not at all statistically defensible. Conversely, considering the minute quantities used from the two separate vessels to obtain these results, the similarities between the results is noteworthy.

4. High frequency sonication may be breaking-up larger particles in the WM-182 and WM-183 tank farm heel slurries. This finding represents useful information regarding ultimate tank heel waste processing. Further investigation of sonication effects is needed and is recommended.

5. Settling rate testing results were fairly consistent in that it appears that most of the mass of solids settle to an agglomerated, yet easily redispersed layer at the bottom; and the $\sim 5 \mu \mathrm{m}$ mode and maximum $30 \mu \mathrm{m}$ size material remains dispersed/suspended in the "clear" layer after about $1 / 2 \mathrm{hr}$. settling time. For future settling rate testing, suspended solids concentration (loading) data should be taken for a detention test analysis as detailed in Perry's. Use of non-fractionated slurry is recommended, however this may require more sample volume.

6. Care must be taken in the use of this particle size data for slurry simulant formulation and in predicting slurry processing and processing equipment performance based on particle size data. Laser-based particle size differs from traditional screen size. Comparison of these tank farm data presented here against laboratory testing data obtained with classical light scattering particle sizing technology will be the ideal case, and is recommended. 


\section{REFERENCES}

1. M. Patterson, Light Duty Utility Arm Deployment in Tank WM-188, INEEL/EXT-99-1302, December 1999.

2. T. A. Batcheller, et al., Remote Laser Diffraction PSD Analyzer, INEEL/EXT-2000-479, June 2000.

3. INEEL Analytical Department Laboratory "Laser Light Scattering PSD Analysis" Method (in Draft), Spring 2000.

4. Terence Allen, Particle Size Measurement, $4^{\text {th }}$ Edition, Chapman and Hall, New York, 1990

5. Horiba Instruction Manual, Laser Scattering Particle Size Distribution Analyzer LA-300, HORIBA, Ltd., Kyoto, Japan, $2^{\text {nd }}$ Edition, September 1998.

6. Personal Conversation, M. Patterson, LDUA Project Manager, June 2000.

7. R. H. Perry, et al., Perry's Chemical Engineers' Handbook, $6^{\text {th }}$ Edition, McGraw-Hill Book Company, New York, 1984. 
Appendix A

\section{Testing Results Data}




\section{Appendix A-1 \\ Particle Size Standards Results Data}

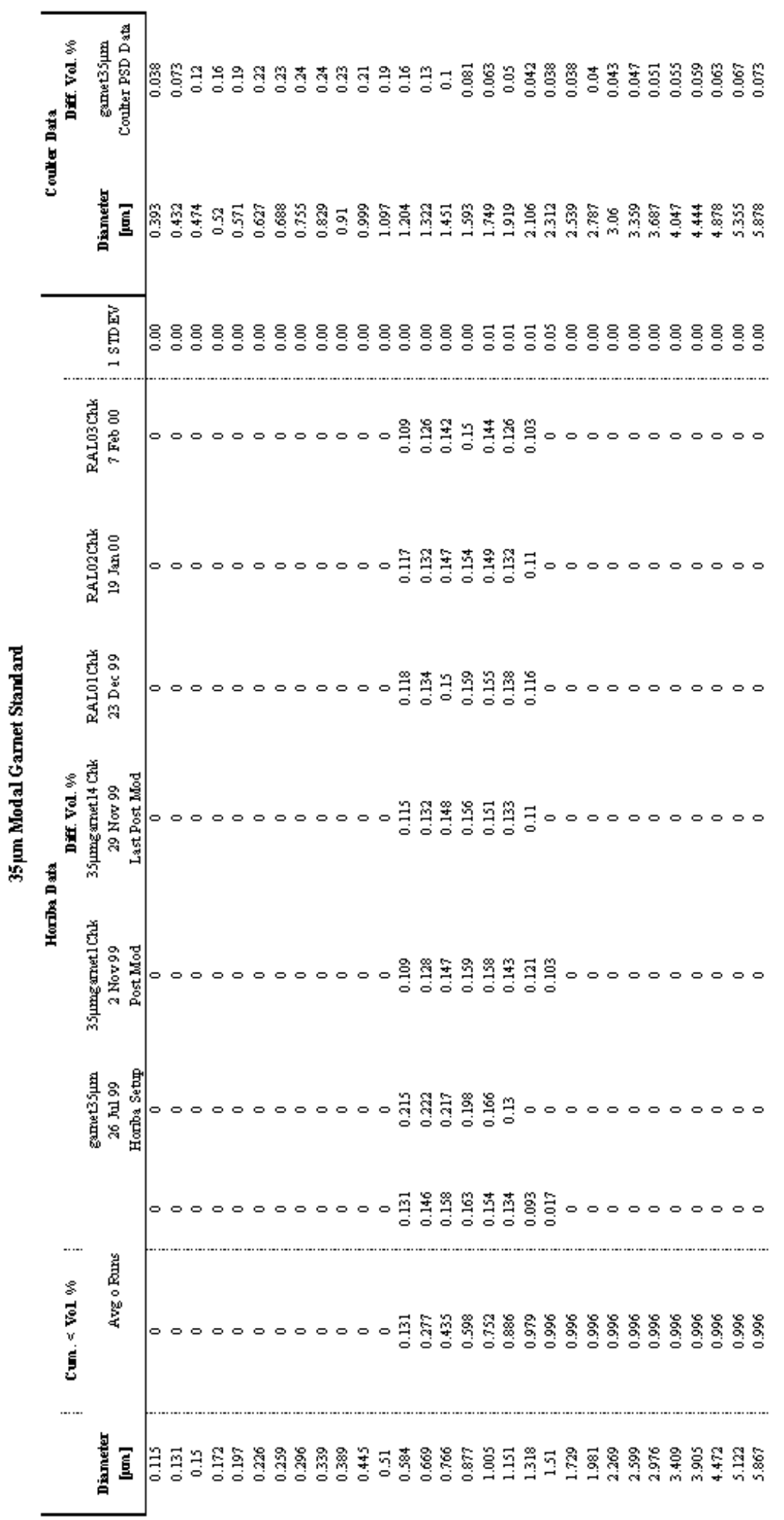




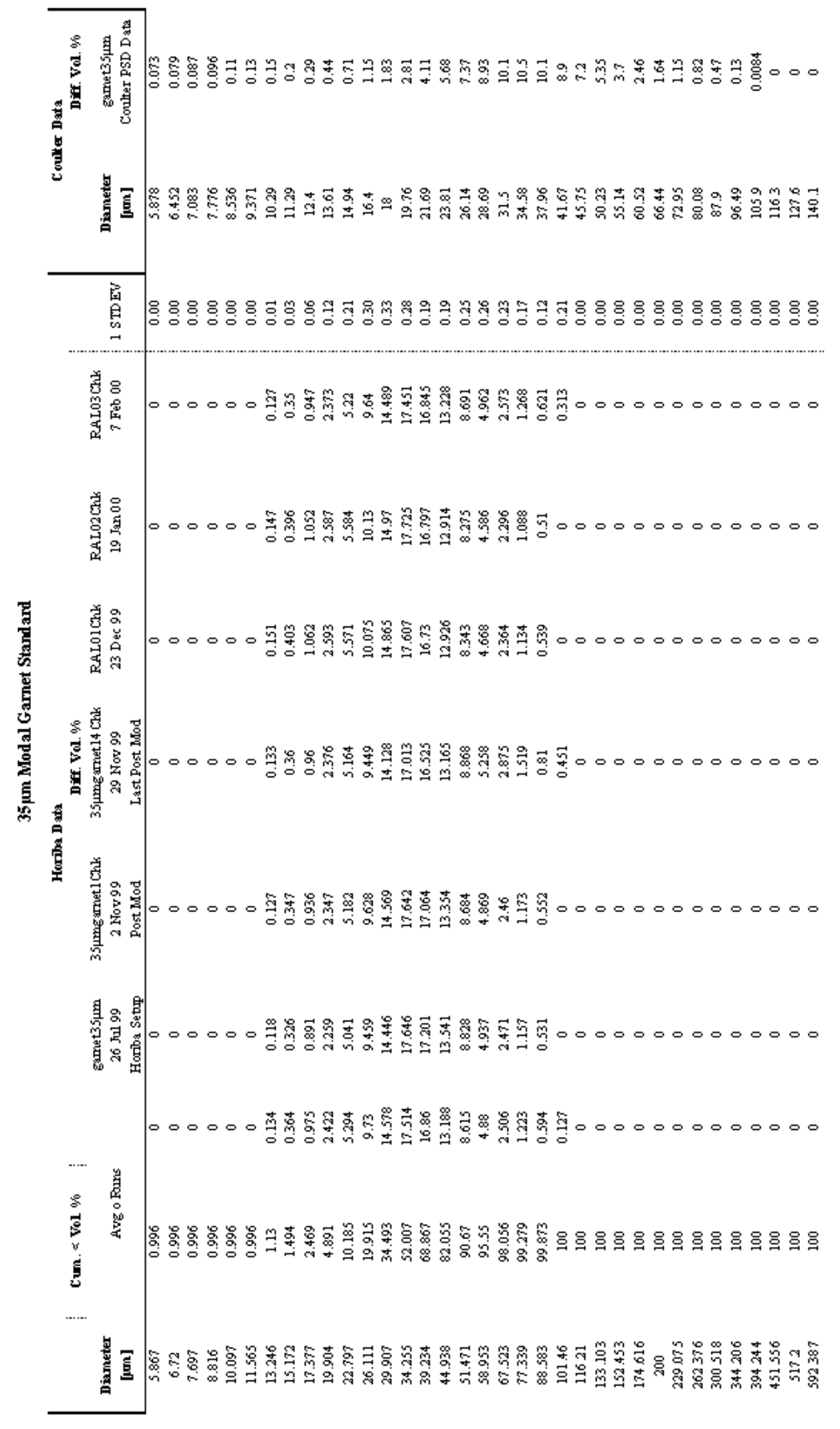




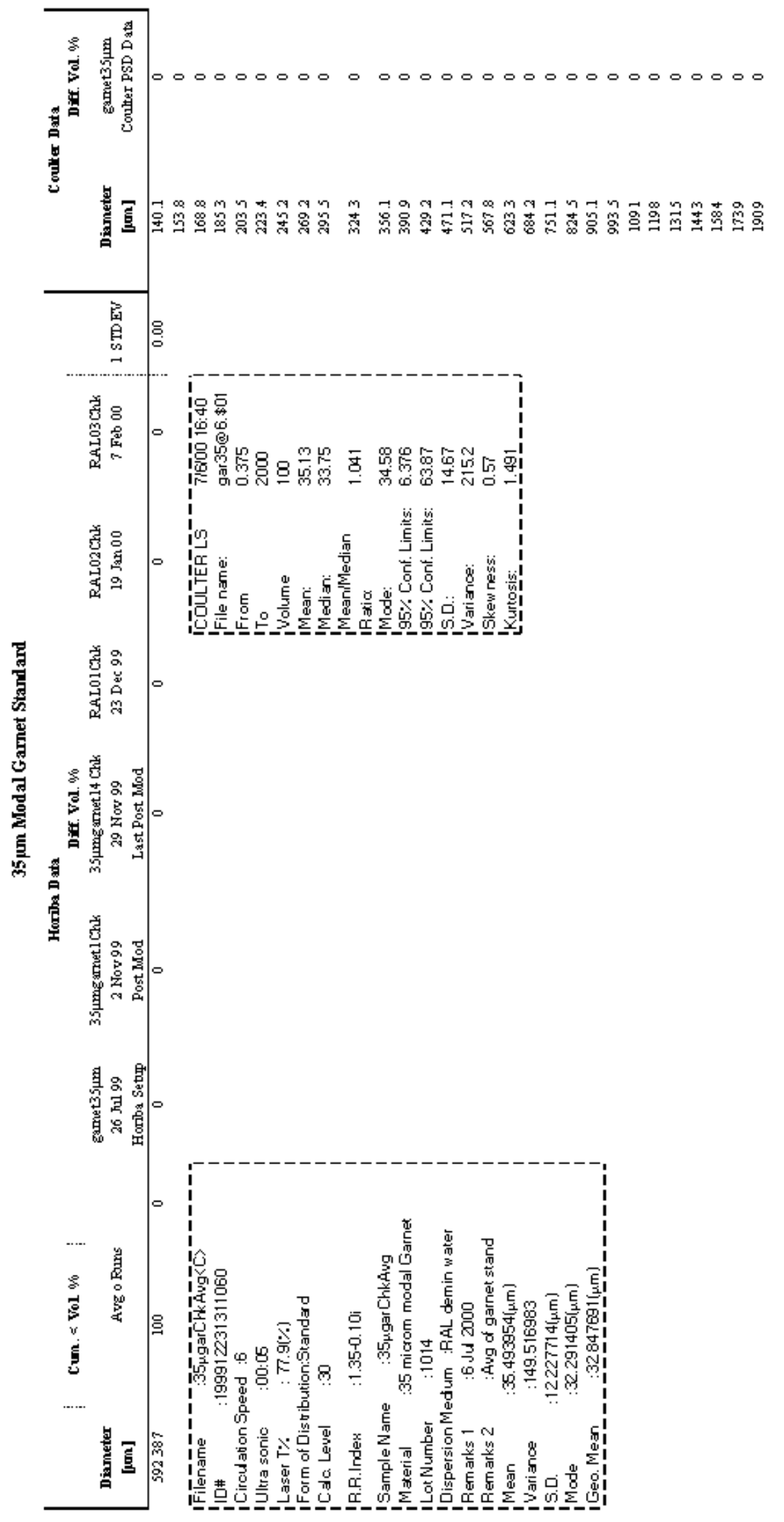


$2 \& 21 \mu \mathrm{m}$ Microsphere Standards Mixture

\begin{tabular}{|c|c|c|}
\hline $\begin{array}{l}\text { Diameter } \\
\qquad[\mu \mathrm{m}]\end{array}$ & Frequency (\%) & Undersize (\%) \\
\hline 0.115 & 0 & 0 \\
\hline 0.131 & 0 & 0 \\
\hline 0.15 & 0 & 0 \\
\hline 0.172 & 0 & 0 \\
\hline 0.197 & 0 & 0 \\
\hline 0.226 & 0 & 0 \\
\hline 0.259 & 0 & 0 \\
\hline 0.296 & 0 & 0 \\
\hline 0.339 & 0 & 0 \\
\hline 0.389 & 0 & 0 \\
\hline 0.445 & 0 & 0 \\
\hline 0.51 & 0 & 0 \\
\hline 0.584 & 0 & 0 \\
\hline 0.669 & 0 & 0 \\
\hline 0.766 & 0 & 0 \\
\hline 0.877 & 0 & 0 \\
\hline 1.005 & 0 & 0 \\
\hline 1.151 & 0 & 0 \\
\hline 1.318 & 0 & 0 \\
\hline 1.51 & 0.472 & 0.472 \\
\hline 1.729 & 0.942 & 1.414 \\
\hline 1.981 & 2.735 & 4.149 \\
\hline 2.269 & 3.125 & 7.274 \\
\hline 2.599 & 0.796 & 8.07 \\
\hline 2.976 & 0.215 & 8.285 \\
\hline 3.409 & 0.121 & 8.406 \\
\hline 3.905 & 0.211 & 8.617 \\
\hline 4.472 & 0.357 & 8.975 \\
\hline 5.122 & 0.27 & 9.244 \\
\hline 5.867 & 0.113 & 9.358 \\
\hline 6.72 & 0 & 9.358 \\
\hline 7.697 & 0 & 9.358 \\
\hline 8.816 & 0 & 9.358 \\
\hline 10.097 & 0.127 & 9.485 \\
\hline 11.565 & 0.298 & 9.783 \\
\hline 13.246 & 1.009 & 10.792 \\
\hline 15.172 & 3.402 & 14.194 \\
\hline 17.377 & 11.39 & 25.584 \\
\hline 19.904 & 24.272 & 49.856 \\
\hline 22.797 & 27.436 & 77.293 \\
\hline 26.111 & 15.345 & 92.638 \\
\hline 29.907 & 5.374 & 98.012 \\
\hline 34.255 & 1.578 & 99.589 \\
\hline 39.234 & 0.411 & 100 \\
\hline 44.938 & 0 & 100 \\
\hline 51.471 & 0 & 100 \\
\hline 58.953 & 0 & 100 \\
\hline 67.523 & 0 & 100 \\
\hline 77.339 & 0 & 100 \\
\hline 88.583 & 0 & 100 \\
\hline 101.46 & 0 & 100 \\
\hline 116.21 & 0 & 100 \\
\hline 133.103 & 0 & 100 \\
\hline 152.453 & 0 & 100 \\
\hline 174.616 & 0 & 100 \\
\hline 200 & 0 & 100 \\
\hline 229.075 & 0 & 100 \\
\hline 262.376 & 0 & 100 \\
\hline 300.518 & 0 & 100 \\
\hline 344.206 & 0 & 100 \\
\hline 394.244 & 0 & 100 \\
\hline 451.556 & 0 & 100 \\
\hline 517.2 & 0 & 100 \\
\hline 592.387 & 0 & 100 \\
\hline
\end{tabular}

\begin{tabular}{|c|c|c|}
\hline :1mmPSChk & Mean & $: 18.969900(\mu \mathrm{m})$ \\
\hline :199911020856019 & Variance & $: 42.976208$ \\
\hline Circulation Speed :4 & S.D. & $6.555624(\mu \mathrm{m})$ \\
\hline Ultra sonic & Mode & $: 20.836029(\mu \mathrm{m})$ \\
\hline |Laser T\% & Geo. Mean & $: 16.468813(\mu \mathrm{m}$ \\
\hline
\end{tabular}

IForm of Distribution:Standard

ICalc. Level : :30

IR.R.Index :PSL-mm

Sample Name :1mmPSChk

Material :mixture polysty micrsphrs

Source :Coulter Standrds; $2 \mu$ \& $21 \mu$ mix

Lot Number :6130 \& 5740 (respectively)

IDispersion Medium :aqua

IRemarks :TAB operator

IRemarks $1 \quad$ :2 Nov 99

IRemarks 2 _ _ polystyrne mixtre of standrds

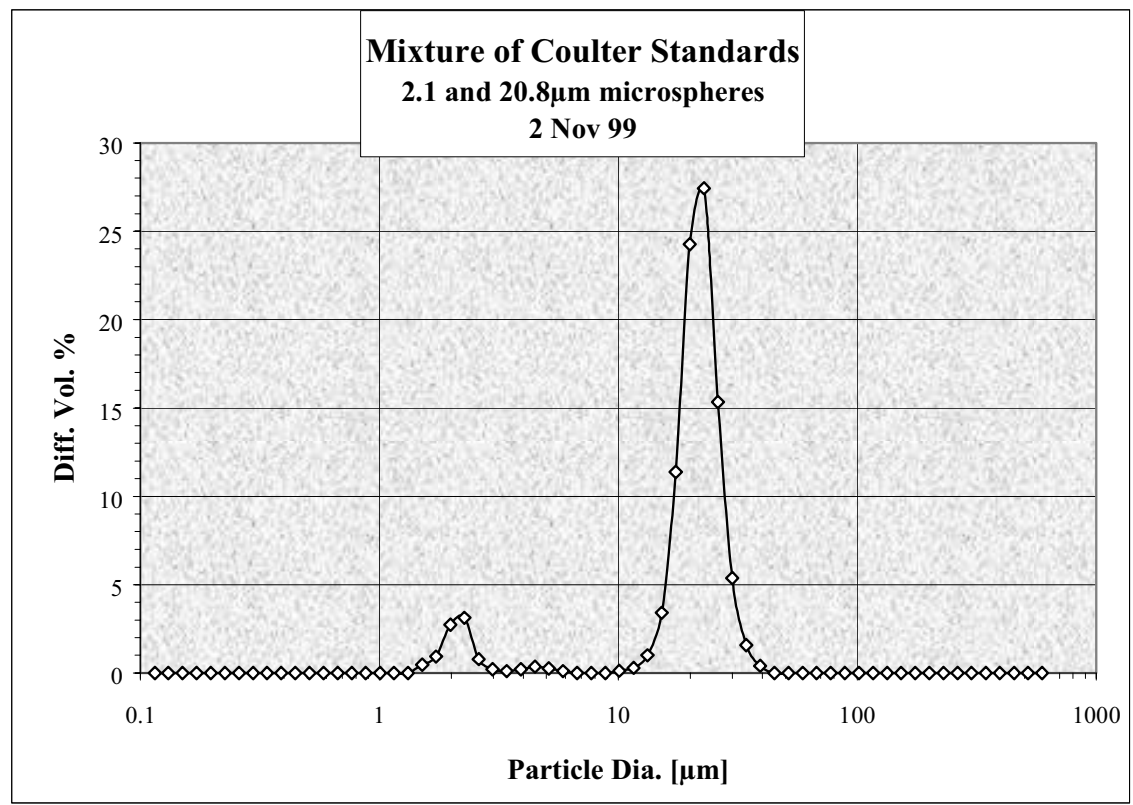




\section{Latron 300LS Standard \\ $0.3 \mu \mathrm{m}$ latex spheres \\ Horiba PSD Analysis @ RAL \\ 11 Apr 2000}

\begin{tabular}{|c|c|c|}
\hline $\begin{array}{c}\text { Diameter } \\
{[\mu \mathrm{m}]}\end{array}$ & Frequency (\%) & Undersize $(\%$ \\
\hline 0.115 & 0 & 0 \\
\hline 0.131 & 0 & 0 \\
\hline 0.15 & 0 & 0 \\
\hline 0.172 & 0 & 0 \\
\hline 0.197 & 0 & 0 \\
\hline 0.226 & 0 & 0 \\
\hline 0.259 & 0 & 0 \\
\hline 0.296 & 15.004 & 15.004 \\
\hline 0.339 & 77.638 & 92.642 \\
\hline 0.389 & 7.358 & 100 \\
\hline 0.445 & 0 & 100 \\
\hline 0.51 & 0 & 100 \\
\hline 0.584 & 0 & 100 \\
\hline 0.669 & 0 & 100 \\
\hline 0.766 & 0 & 100 \\
\hline 0.877 & 0 & 100 \\
\hline 1.005 & 0 & 100 \\
\hline 1.151 & 0 & 100 \\
\hline 1.318 & 0 & 100 \\
\hline 1.51 & 0 & 100 \\
\hline 1.729 & 0 & 100 \\
\hline 1.981 & 0 & 100 \\
\hline 2.269 & 0 & 100 \\
\hline 2.599 & 0 & 100 \\
\hline 2.976 & 0 & 100 \\
\hline 3.409 & 0 & 100 \\
\hline 3.905 & 0 & 100 \\
\hline 4.472 & 0 & 100 \\
\hline 5.122 & 0 & 100 \\
\hline 5.867 & 0 & 100 \\
\hline 6.72 & 0 & 100 \\
\hline 7.697 & 0 & 100 \\
\hline 8.816 & 0 & 100 \\
\hline 10.097 & 0 & 100 \\
\hline 11.565 & 0 & 100 \\
\hline 13.246 & 0 & 100 \\
\hline 15.172 & 0 & 100 \\
\hline 17.377 & 0 & 100 \\
\hline 19.904 & 0 & 100 \\
\hline 22.797 & 0 & 100 \\
\hline 26.111 & 0 & 100 \\
\hline 29.907 & 0 & 100 \\
\hline 34.255 & 0 & 100 \\
\hline 39.234 & 0 & 100 \\
\hline 44.938 & 0 & 100 \\
\hline 51.471 & 0 & 100 \\
\hline 58.953 & 0 & 100 \\
\hline 67.523 & 0 & 100 \\
\hline 77.339 & 0 & 100 \\
\hline 88.583 & 0 & 100 \\
\hline 101.46 & 0 & 100 \\
\hline 116.21 & 0 & 100 \\
\hline 133.103 & 0 & 100 \\
\hline 152.453 & 0 & 100 \\
\hline 174.616 & 0 & 100 \\
\hline 200 & 0 & 100 \\
\hline 229.075 & 0 & 100 \\
\hline 262.376 & 0 & 100 \\
\hline 300.518 & 0 & 100 \\
\hline 344.206 & 0 & 100 \\
\hline 394.244 & 0 & 100 \\
\hline 451.556 & 0 & 100 \\
\hline
\end{tabular}

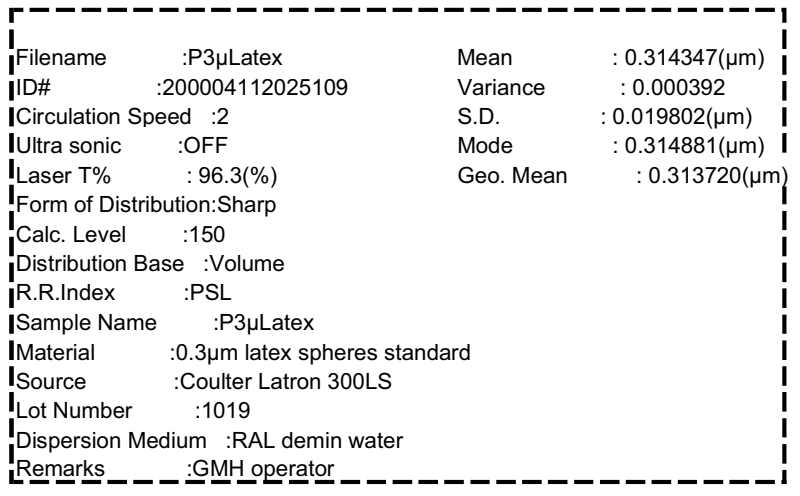

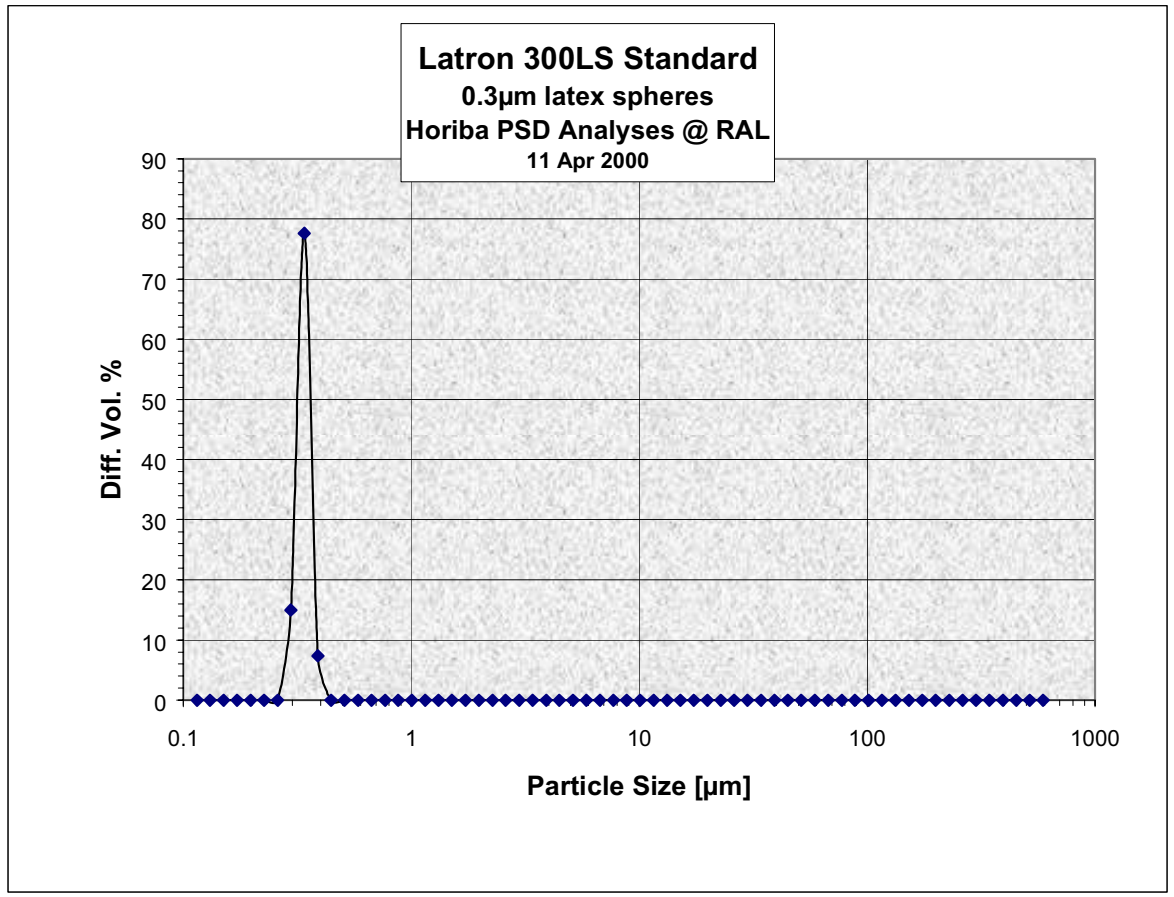




\section{Appendix A-2 Tank Farm Slurry Sample PSD Results Data}

WM182 PSD Analysis

WM182\#4 sonicated run

Diff. Frequency $(\%)$

\begin{tabular}{|c|c|c|c|c|c|}
\hline Diameter & Avg o Runs & Run 06 & Run 07 & Run 08 & 1 STDEV \\
\hline 0.115 & 0 & 0 & 0 & 0 & 0.00 \\
\hline 0.131 & 0 & 0 & 0 & 0 & 0.00 \\
\hline 0.15 & 0 & 0 & 0 & 0 & 0.00 \\
\hline 0.172 & 0 & 0 & 0 & 0 & 0.00 \\
\hline 0.197 & 0 & 0 & 0 & 0 & 0.00 \\
\hline 0.226 & 0 & 0 & 0 & 0 & 0.00 \\
\hline 0.259 & 0 & 0 & 0 & 0 & 0.00 \\
\hline 0.296 & 0 & 0 & 0 & 0 & 0.00 \\
\hline 0.339 & 0 & 0 & 0 & 0 & 0.00 \\
\hline 0.389 & 0 & 0 & 0 & 0 & 0.00 \\
\hline 0.445 & 0.114 & 0 & 0.343 & 0 & 0.20 \\
\hline 0.51 & 0.14 & 0 & 0.419 & 0 & 0.24 \\
\hline 0.584 & 0.342 & 0.248 & 0.517 & 0.261 & 0.15 \\
\hline 0.669 & 0.462 & 0.366 & 0.636 & 0.385 & 0.15 \\
\hline 0.766 & 0.612 & 0.515 & 0.778 & 0.543 & 0.14 \\
\hline 0.877 & 0.787 & 0.687 & 0.946 & 0.728 & 0.14 \\
\hline 1.005 & 0.949 & 0.846 & 1.101 & 0.9 & 0.13 \\
\hline 1.151 & 1.121 & 1.01 & 1.278 & 1.074 & 0.14 \\
\hline 1.318 & 1.297 & 1.175 & 1.466 & 1.25 & 0.15 \\
\hline 1.51 & 1.469 & 1.343 & 1.629 & 1.435 & 0.15 \\
\hline 1.729 & 1.664 & 1.524 & 1.837 & 1.63 & 0.16 \\
\hline 1.981 & 1.877 & 1.718 & 2.076 & 1.836 & 0.18 \\
\hline 2.269 & 2.059 & 1.889 & 2.273 & 2.014 & 0.20 \\
\hline 2.599 & 2.336 & 2.153 & 2.565 & 2.289 & 0.21 \\
\hline 2.976 & 2.66 & 2.463 & 2.905 & 2.613 & 0.22 \\
\hline 3.409 & 3.126 & 2.917 & 3.378 & 3.084 & 0.23 \\
\hline 3.905 & 3.618 & 3.405 & 3.859 & 3.59 & 0.23 \\
\hline 4.472 & 4.094 & 3.889 & 4.307 & 4.085 & 0.21 \\
\hline 5.122 & 4.556 & 4.37 & 4.727 & 4.571 & 0.18 \\
\hline 5.867 & 4.964 & 4.807 & 5.081 & 5.004 & 0.14 \\
\hline 6.72 & 5.386 & 5.264 & 5.442 & 5.451 & 0.11 \\
\hline 7.697 & 5.754 & 5.677 & 5.74 & 5.845 & 0.08 \\
\hline 8.816 & 5.964 & 5.947 & 5.875 & 6.072 & 0.10 \\
\hline 10.097 & 6.139 & 6.184 & 5.971 & 6.263 & 0.15 \\
\hline 11.565 & 6.152 & 6.265 & 5.908 & 6.284 & 0.21 \\
\hline 13.246 & 5.977 & 6.157 & 5.668 & 6.107 & 0.27 \\
\hline 15.172 & 5.603 & 5.845 & 5.243 & 5.721 & 0.32 \\
\hline 17.377 & 5.041 & 5.333 & 4.65 & 5.14 & 0.35 \\
\hline 19.904 & 4.327 & 4.653 & 3.927 & 4.402 & 0.37 \\
\hline 22.797 & 3.525 & 3.864 & 3.138 & 3.574 & 0.37 \\
\hline 26.111 & 2.713 & 3.043 & 2.357 & 2.739 & 0.34 \\
\hline 29.907 & 1.966 & 2.269 & 1.656 & 1.974 & 0.31 \\
\hline 34.255 & 1.34 & 1.601 & 1.083 & 1.336 & 0.26 \\
\hline 39.234 & 0.859 & 1.072 & 0.658 & 0.848 & 0.21 \\
\hline 44.938 & 0.521 & 0.685 & 0.37 & 0.508 & 0.16 \\
\hline 51.471 & 0.301 & 0.42 & 0.194 & 0.288 & 0.11 \\
\hline 58.953 & 0.135 & 0.25 & 0 & 0.156 & 0.13 \\
\hline 67.523 & 0.049 & 0.147 & 0 & 0 & 0.08 \\
\hline 77.339 & 0 & 0 & 0 & 0 & 0.00 \\
\hline 88.583 & 0 & 0 & 0 & 0 & 0.00 \\
\hline 101.46 & 0 & 0 & 0 & 0 & 0.00 \\
\hline 116.21 & 0 & 0 & 0 & 0 & 0.00 \\
\hline 133.103 & 0 & 0 & 0 & 0 & 0.00 \\
\hline 152.453 & 0 & 0 & 0 & 0 & 0.00 \\
\hline 174.616 & 0 & 0 & 0 & 0 & 0.00 \\
\hline 200 & 0 & 0 & 0 & 0 & 0.00 \\
\hline 229.075 & 0 & 0 & 0 & 0 & 0.00 \\
\hline 262.376 & 0 & 0 & 0 & 0 & 0.00 \\
\hline 300.518 & 0 & 0 & 0 & 0 & 0.00 \\
\hline 344.206 & 0 & 0 & 0 & 0 & 0.00 \\
\hline 394.244 & 0 & 0 & 0 & 0 & 0.00 \\
\hline 451.556 & 0 & 0 & 0 & 0 & 0.00 \\
\hline 517.2 & 0 & 0 & 0 & 0 & 0.00 \\
\hline 592.387 & 0 & 0 & 0 & 0 & 0.00 \\
\hline
\end{tabular}

WM182\#4 non-sonicated runs Diff. Frequency \%

Avg o Runs Run 01 Run 02 Run 03 Run 04 Run 05 1 STDEV Run 01 $\begin{array}{lllllllr}0 & 0 & 0 & 0 & 0 & 0 & 0.00 & 0\end{array}$

$\begin{array}{lllllll}0 & 0 & 0 & 0 & 0 & 0 & 0.00 \\ 0 & 0 & 0 & 0 & 0 & 0 & 0.00 \\ 0 & 0 & 0 & 0 & 0 & 0 & 0.00 \\ 0 & 0 & 0 & 0 & 0 & 0 & 0.00 \\ 0 & 0 & 0 & 0 & 0 & 0 & 0.00 \\ 0 & 0 & 0 & 0 & 0 & 0 & 0.00 \\ 0 & 0 & 0 & 0 & 0 & 0 & 0.00 \\ 0 & 0 & 0 & 0 & 0 & 0 & 0.00 \\ 0 & 0 & 0 & 0 & 0 & 0 & 0.00 \\ 0 & 0 & 0 & 0 & 0 & 0 & 0.00\end{array}$

$\begin{array}{cccccccc}0 & 0 & 0 & 0 & 0 & 0 & 0.00 & 0.369 \\ 0.185 & 0.189 & 0.173 & 0.137 & 0.271 & 0.157 & 0.05 & 0.567\end{array}$

$\begin{array}{llllllll}0.239 & 0.247 & 0.229 & 0.187 & 0.322 & 0.21 & 0.05 & 0.853\end{array}$

$\begin{array}{llllllll}0.307 & 0.32 & 0.3 & 0.251 & 0.387 & 0.276 & 0.05 & 1.238\end{array}$

$\begin{array}{llllllll}0.389 & 0.408 & 0.385 & 0.327 & 0.469 & 0.355 & 0.05 & 1.701 \\ 0.469 & 0.494 & 0.466 & 0.4 & 0.555 & 0.43 & 0.06 & 2.091\end{array}$

$\begin{array}{llllllll}0.469 & 0.494 & 0.466 & 0.4 & 0.555 & 0.43 & 0.06 & 2.091\end{array}$

$\begin{array}{llllllll}0.556 & 0.585 & 0.552 & 0.476 & 0.659 & 0.509 & 0.07 & 2.429\end{array}$

$\begin{array}{llllllll}0.652 & 0.685 & 0.646 & 0.559 & 0.774 & 0.596 & 0.08 & 2.706\end{array}$

$\begin{array}{llllllll}0.754 & 0.793 & 0.749 & 0.653 & 0.881 & 0.692 & 0.09 & 2.977\end{array}$

$\begin{array}{llllllll}0.873 & 0.917 & 0.867 & 0.76 & 1.018 & 0.803 & 0.10 & 3.116\end{array}$

$\begin{array}{llllllll}1.008 & 1.057 & 1.001 & 0.88 & 1.176 & 0.927 & 0.12 & 3.201\end{array}$

$\begin{array}{llllllll}1.133 & 1.185 & 1.125 & 0.994 & 1.316 & 1.043 & 0.13 & 3.199\end{array}$

$\begin{array}{llllllll}1.307 & 1.364 & 1.3 & 1.159 & 1.505 & 1.208 & 0.14 & 3.245\end{array}$

$\begin{array}{llllllll}1.506 & 1.567 & 1.5 & 1.346 & 1.72 & 1.396 & 0.15 & 3.362\end{array}$

$\begin{array}{llllllll}1.779 & 1.846 & 1.776 & 1.612 & 2.004 & 1.657 & 0.16 & 3.492\end{array}$

$\begin{array}{llllllll}2.063 & 2.134 & 2.065 & 1.899 & 2.286 & 1.934 & 0.16 & 3.552\end{array}$

$\begin{array}{llllllll}2.329 & 2.401 & 2.336 & 2.177 & 2.534 & 2.197 & 0.15 & 3.541\end{array}$

$\begin{array}{llllllll}2.568 & 2.641 & 2.581 & 2.433 & 2.747 & 2.436 & 0.14 & 3.509\end{array}$

$\begin{array}{llllllll}2.727 & 2.805 & 2.749 & 2.605 & 2.879 & 2.599 & 0.12 & 3.571\end{array}$

$\begin{array}{llllllll}2.906 & 2.988 & 2.936 & 2.796 & 3.029 & 2.78 & 0.11 & 3.639\end{array}$

$\begin{array}{llllllll}3.05 & 3.14 & 3.09 & 2.947 & 3.145 & 2.927 & 0.11 & 3.749\end{array}$

$\begin{array}{llllllll}3.152 & 3.257 & 3.203 & 3.043 & 3.229 & 3.03 & 0.11 & 3.96\end{array}$

$\begin{array}{llllllll}3.267 & 3.385 & 3.329 & 3.153 & 3.323 & 3.144 & 0.11 & 4.131\end{array}$

$\begin{array}{llllllll}3.361 & 3.496 & 3.434 & 3.238 & 3.401 & 3.236 & 0.12 & 4.287\end{array}$

$\begin{array}{lllllll}3.442 & 3.595 & 3.524 & 3.308 & 3.471 & 3.313 & 0.13\end{array}$

$\begin{array}{llllllll}3.517 & 3.687 & 3.605 & 3.371 & 3.538 & 3.382 & 0.14 & 4.394\end{array}$

$\begin{array}{llllllll}3.589 & 3.777 & 3.681 & 3.437 & 3.605 & 3.447 & 0.15 & 4.262\end{array}$

$\begin{array}{llllllll}3.66 & 3.86 & 3.749 & 3.51 & 3.672 & 3.51 & 0.15 & 3.971\end{array}$

$\begin{array}{llllllll}3.727 & 3.934 & 3.806 & 3.592 & 3.734 & 3.57 & 0.15 & 3.533\end{array}$

$\begin{array}{llllllll}3.786 & 3.991 & 3.848 & 3.683 & 3.786 & 3.625 & 0.14 & 2.991\end{array}$

$\begin{array}{llllllll}3.833 & 4.022 & 3.869 & 3.783 & 3.819 & 3.672 & 0.13 & 2.406\end{array}$

$\begin{array}{llllllll}3.86 & 4.019 & 3.865 & 3.886 & 3.824 & 3.707 & 0.11 & 1.844\end{array}$

$\begin{array}{llllllll}3.86 & 3.969 & 3.831 & 3.984 & 3.79 & 3.725 & 0.11 & 1.354\end{array}$

$\begin{array}{llllllll}3.822 & 3.862 & 3.76 & 4.062 & 3.706 & 3.72 & 0.15 & 0.958\end{array}$

$\begin{array}{llllllll}3.739 & 3.694 & 3.649 & 4.1 & 3.565 & 3.685 & 0.21 & 0.66\end{array}$

$\begin{array}{llllllll}3.604 & 3.464 & 3.497 & 4.074 & 3.367 & 3.617 & 0.28 & 0.448 \\ 3.414 & 3.179 & 3.305 & 3.959 & 3.115 & 3.512 & 0.34 & 0.304\end{array}$

$\begin{array}{llllllll}3.414 & 3.179 & 3.305 & 3.959 & 3.115 & 3.512 & 0.34 & 0.304\end{array}$

$\begin{array}{lllllll}3.171 & 2.853 & 3.075 & 3.736 & 2.823 & 3.368 & 0.38\end{array}$

$\begin{array}{lllllll}2.88 & 2.503 & 2.808 & 3.398 & 2.506 & 3.186 & 0.40\end{array}$

$\begin{array}{lllllll}2.553 & 2.148 & 2.508 & 2.959 & 2.182 & 2.967 & 0.40\end{array}$

$\begin{array}{lllllll}2.204 & 1.806 & 2.18 & 2.454 & 1.867 & 2.712 & 0.39\end{array}$

$\begin{array}{lllllll}1.852 & 1.488 & 1.839 & 1.937 & 1.572 & 2.425 & 0.37\end{array}$

$\begin{array}{lllllll}1.52 & 1.205 & 1.508 & 1.466 & 1.304 & 2.117 & 0.36\end{array}$

$\begin{array}{lllllll}0.844 & 0.67 & 0.838 & 0.814 & 0.725 & 1.176 & 0.20\end{array}$

$\begin{array}{lllllll}0.469 & 0.372 & 0.465 & 0.452 & 0.403 & 0.654 & 0.11\end{array}$

$\begin{array}{lllllll}0.073 & 0 & 0 & 0 & 0 & 0.363 & 0.16\end{array}$

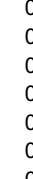




\begin{tabular}{|c|c|}
\hline Filename & :9911082WM182\#4-savg<C> \\
\hline ID\# & :200003081413108 \\
\hline \multicolumn{2}{|c|}{ Circulation Speed $: 6$} \\
\hline Ultra sonic & $: 00: 13$ \\
\hline Laser T\% & : 87.2(\%) \\
\hline \multicolumn{2}{|c|}{ Form of Distribution:Standard } \\
\hline Calc. Level & :30 \\
\hline R.R.Index & $: 1.35-0.10 i$ \\
\hline Material & :WM182\#4 \\
\hline Source & $:$ \\
\hline \multicolumn{2}{|l|}{ Lot Number } \\
\hline \multicolumn{2}{|c|}{ Dispersion Medium :RAL demin water + usonic } \\
\hline Remarks & :GMH operator \\
\hline Remarks 1 & :8 Mar 2000 \\
\hline Remarks 2 & :Avg of Runs 06 to 08 \\
\hline Mean & $: 10.023014(\mu \mathrm{m})$ \\
\hline Variance & :69.660103 \\
\hline S.D. & : 8.346263( $\mu \mathrm{m})$ \\
\hline Mode & $: 10.786839(\mu \mathrm{m})$ \\
\hline Geo. Mean & : 6.977938( $\mu \mathrm{m})$ \\
\hline
\end{tabular}

\begin{tabular}{|c|c|}
\hline Filename & :9911082WM182\#4-nsavg<C> \\
\hline ID\# & :199912231337061 - - \\
\hline \multicolumn{2}{|c|}{ Circulation Speed $: 6$} \\
\hline Ultra sonic & :OFF \\
\hline Laser T\% & : 73.8(\%) \\
\hline \multicolumn{2}{|c|}{ Form of Distribution:Standard } \\
\hline Calc. Level & :30 \\
\hline R.R.Index & $: 1.35-0.10 i$ \\
\hline Material & :WM182 \#4 Slurry \\
\hline Source & $:$ \\
\hline \multicolumn{2}{|l|}{ Lot Number } \\
\hline \multicolumn{2}{|c|}{ Dispersion Medium :RAL demin water } \\
\hline Remarks & :GMH/TAB operators \\
\hline Remarks 1 & :23 Dec 99 \\
\hline Remarks 2 & :Avg of Runs 01 to 05 \\
\hline Mean & $: 33.539398(\mu \mathrm{m})$ \\
\hline Variance & :1352.909424 \\
\hline S.D. & $: 36.781918(\mu \mathrm{m})$ \\
\hline Mode & $: 32.022137(\mu \mathrm{m})$ \\
\hline Geo. Mean & $: 17.479652(\mu \mathrm{m})$ \\
\hline
\end{tabular}

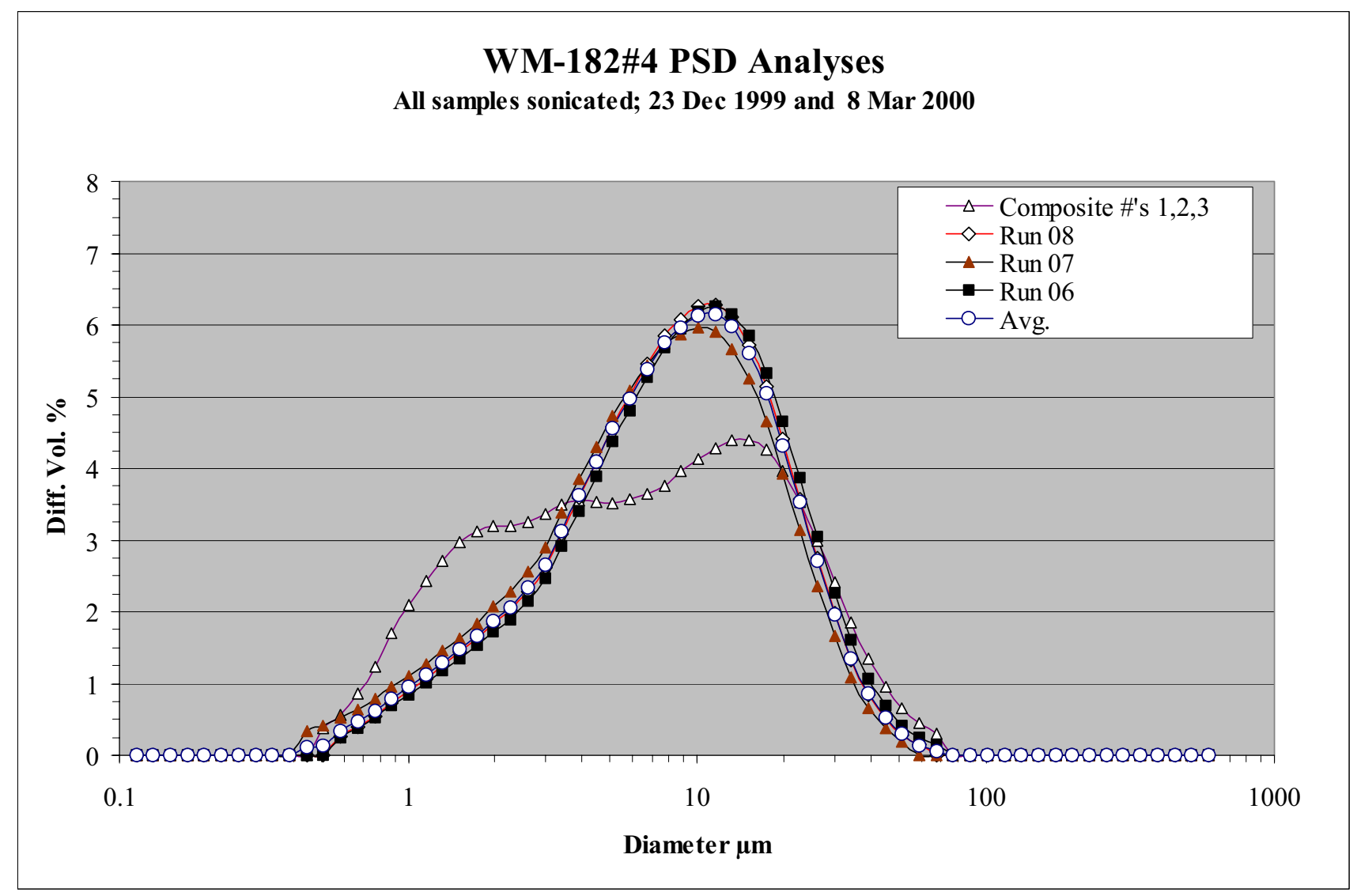




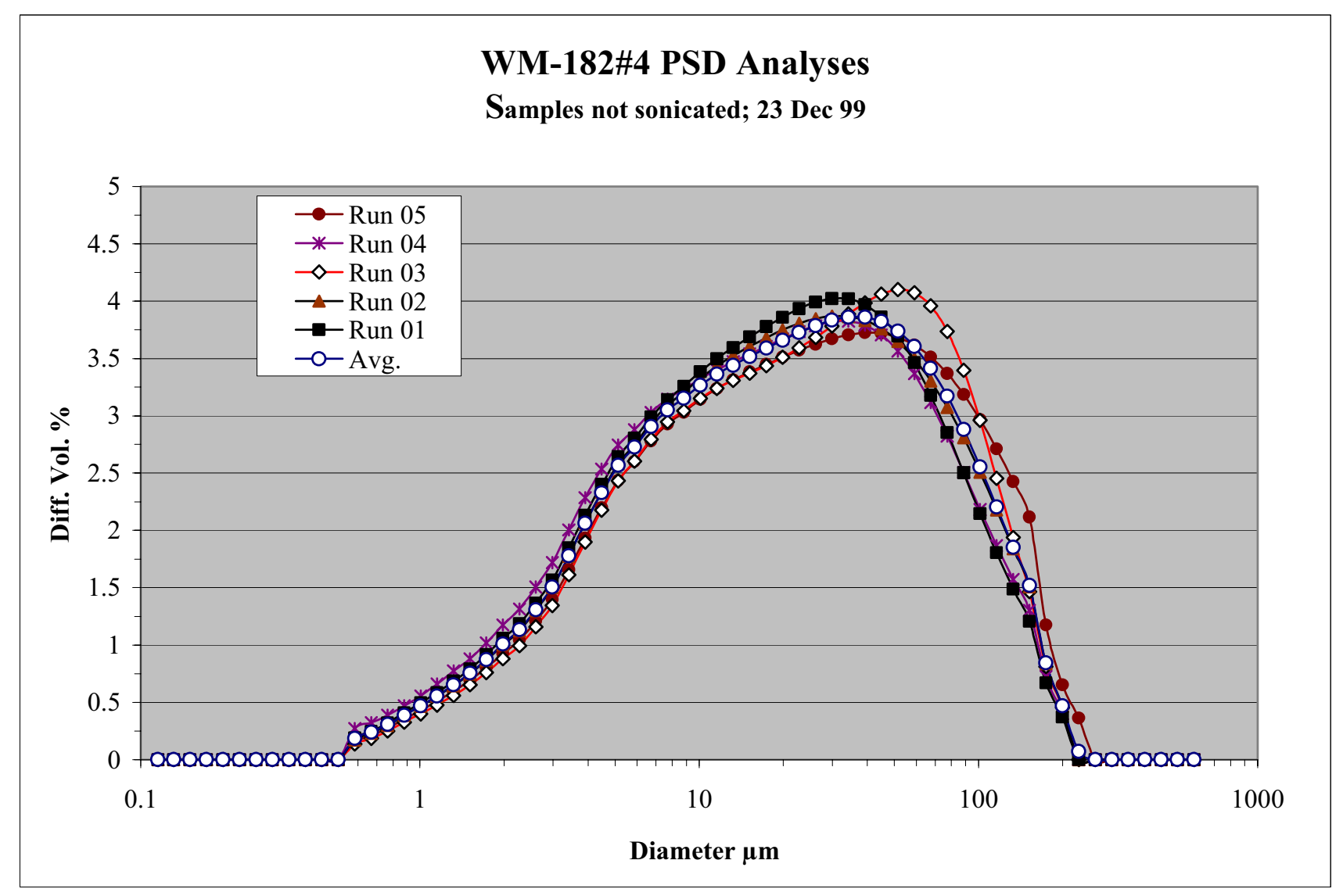


WM183\#3 PSD Analysis

WM183\#3 sonicated runs

Diff. Frequency (\%)

\begin{tabular}{|c|c|c|c|c|c|c|c|c|c|c|}
\hline & & & & & & & & & & \\
\hline Diameter & Avg o Runs & Run 01 & Run 04 & Run 05 & 1 STDEV & Avg o Runs & Run 02 & Run 03 & Run 06 & 1 STDEV \\
\hline 0.115 & 0 & 0 & 0 & 0 & 0 & 0 & 0 & 0 & 0 & 0 \\
\hline 0.131 & 0 & 0 & 0 & 0 & 0.000 & 0 & 0 & 0 & 0 & 0.000 \\
\hline 0.15 & 0 & 0 & 0 & 0 & 0.000 & 0 & 0 & 0 & 0 & 0.000 \\
\hline 0.172 & 0 & 0 & 0 & 0 & 0.000 & 0 & 0 & 0 & 0 & 0.000 \\
\hline 0.197 & 0 & 0 & 0 & 0 & 0.000 & 0 & 0 & 0 & 0 & 0.000 \\
\hline 0.226 & 0 & 0 & 0 & 0 & 0.000 & 0 & 0 & 0 & 0 & 0.000 \\
\hline 0.259 & 0 & 0 & 0 & 0 & 0.000 & 0 & 0 & 0 & 0 & 0.000 \\
\hline 0.296 & 0 & 0 & 0 & 0 & 0.000 & 0 & 0 & 0 & 0 & 0.000 \\
\hline 0.339 & 0 & 0 & 0 & 0 & 0.000 & 0 & 0 & 0 & 0 & 0.000 \\
\hline 0.389 & 0 & 0 & 0 & 0 & 0.000 & 0 & 0 & 0 & 0 & 0.000 \\
\hline 0.445 & 0 & 0 & 0 & 0 & 0.000 & 0 & 0 & 0 & 0 & 0.000 \\
\hline 0.51 & 0 & 0 & 0 & 0 & 0.000 & 0 & 0 & 0 & 0 & 0.000 \\
\hline 0.584 & 0.055 & 0 & 0 & 0.164 & 0.095 & 0 & 0 & 0 & 0 & 0.000 \\
\hline 0.669 & 0.15 & 0.11 & 0.131 & 0.21 & 0.053 & 0 & 0 & 0 & 0 & 0.000 \\
\hline 0.766 & 0.212 & 0.162 & 0.194 & 0.279 & 0.060 & 0.128 & 0.13 & 0.134 & 0.12 & 0.007 \\
\hline 0.877 & 0.298 & 0.237 & 0.282 & 0.375 & 0.070 & 0.184 & 0.185 & 0.192 & 0.174 & 0.009 \\
\hline 1.005 & 0.404 & 0.329 & 0.388 & 0.494 & 0.084 & 0.25 & 0.252 & 0.261 & 0.239 & 0.011 \\
\hline 1.151 & 0.53 & 0.438 & 0.511 & 0.642 & 0.103 & 0.329 & 0.329 & 0.342 & 0.314 & 0.014 \\
\hline 1.318 & 0.674 & 0.563 & 0.649 & 0.81 & 0.125 & 0.418 & 0.417 & 0.436 & 0.402 & 0.017 \\
\hline 1.51 & 0.839 & 0.716 & 0.815 & 0.986 & 0.137 & 0.527 & 0.524 & 0.549 & 0.509 & 0.020 \\
\hline 1.729 & 1 & 0.865 & 0.971 & 1.165 & 0.152 & 0.639 & 0.634 & 0.664 & 0.619 & 0.023 \\
\hline 1.981 & 1.166 & 1.02 & 1.128 & 1.351 & 0.169 & 0.759 & 0.752 & 0.788 & 0.736 & 0.027 \\
\hline 2.269 & 1.319 & 1.168 & 1.273 & 1.516 & 0.179 & 0.876 & 0.868 & 0.909 & 0.852 & 0.029 \\
\hline 2.599 & 1.497 & 1.342 & 1.444 & 1.706 & 0.188 & 1.027 & 1.017 & 1.063 & 1.001 & 0.032 \\
\hline 2.976 & 1.691 & 1.532 & 1.63 & 1.913 & 0.198 & 1.193 & 1.182 & 1.232 & 1.166 & 0.034 \\
\hline 3.409 & 1.96 & 1.792 & 1.892 & 2.196 & 0.210 & 1.435 & 1.423 & 1.476 & 1.406 & 0.037 \\
\hline 3.905 & 2.254 & 2.075 & 2.189 & 2.499 & 0.219 & 1.712 & 1.7 & 1.753 & 1.684 & 0.036 \\
\hline 4.472 & 2.573 & 2.375 & 2.52 & 2.824 & 0.229 & 2.016 & 2.004 & 2.053 & 1.989 & 0.033 \\
\hline 5.122 & 2.931 & 2.698 & 2.904 & 3.19 & 0.247 & 2.342 & 2.332 & 2.373 & 2.319 & 0.028 \\
\hline 5.867 & 3.392 & 3.074 & 3.424 & 3.677 & 0.303 & 2.663 & 2.656 & 2.686 & 2.648 & 0.020 \\
\hline 6.72 & 3.887 & 3.481 & 3.987 & 4.193 & 0.366 & 3 & 2.995 & 3.012 & 2.993 & 0.010 \\
\hline 7.697 & 4.447 & 3.927 & 4.64 & 4.774 & 0.455 & 3.317 & 3.314 & 3.317 & 3.321 & 0.004 \\
\hline 8.816 & 5.053 & 4.416 & 5.357 & 5.386 & 0.552 & 3.539 & 3.534 & 3.526 & 3.555 & 0.015 \\
\hline 10.097 & 5.667 & 4.926 & 6.085 & 5.989 & 0.643 & 3.767 & 3.762 & 3.742 & 3.797 & 0.028 \\
\hline 11.565 & 6.228 & 5.427 & 6.749 & 6.508 & 0.704 & 3.927 & 3.92 & 3.89 & 3.97 & 0.040 \\
\hline 13.246 & 6.663 & 5.882 & 7.248 & 6.86 & 0.704 & 4.014 & 4.005 & 3.969 & 4.069 & 0.051 \\
\hline 15.172 & 6.892 & 6.245 & 7.472 & 6.958 & 0.616 & 4.035 & 4.023 & 3.984 & 4.098 & 0.058 \\
\hline 17.377 & 6.847 & 6.46 & 7.336 & 6.746 & 0.447 & 4.002 & 3.987 & 3.949 & 4.07 & 0.062 \\
\hline 19.904 & 6.5 & 6.475 & 6.813 & 6.211 & 0.302 & 3.93 & 3.913 & 3.879 & 3.999 & 0.062 \\
\hline 22.797 & 5.872 & 6.251 & 5.955 & 5.41 & 0.427 & 3.836 & 3.817 & 3.79 & 3.9 & 0.057 \\
\hline 26.111 & 5.04 & 5.784 & 4.887 & 4.448 & 0.681 & 3.733 & 3.715 & 3.696 & 3.789 & 0.049 \\
\hline 29.907 & 4.108 & 5.106 & 3.765 & 3.454 & 0.878 & 3.635 & 3.619 & 3.608 & 3.677 & 0.037 \\
\hline 34.255 & 3.185 & 4.286 & 2.731 & 2.539 & 0.958 & 3.545 & 3.533 & 3.532 & 3.571 & 0.022 \\
\hline 39.234 & 2.354 & 3.413 & 1.874 & 1.776 & 0.918 & 3.467 & 3.459 & 3.468 & 3.475 & 0.008 \\
\hline 44.938 & 1.665 & 2.579 & 1.225 & 1.19 & 0.792 & 3.398 & 3.395 & 3.413 & 3.386 & 0.014 \\
\hline 51.471 & 1.131 & 1.852 & 0.772 & 0.77 & 0.624 & 3.331 & 3.334 & 3.358 & 3.301 & 0.029 \\
\hline 58.953 & 0.744 & 1.271 & 0.473 & 0.487 & 0.457 & 3.261 & 3.271 & 3.297 & 3.216 & 0.041 \\
\hline 67.523 & 0.477 & 0.839 & 0.287 & 0.305 & 0.314 & 3.182 & 3.199 & 3.221 & 3.127 & 0.049 \\
\hline 77.339 & 0.18 & 0.54 & 0 & 0 & 0.312 & 3.087 & 3.111 & 3.122 & 3.029 & 0.051 \\
\hline 88.583 & 0.114 & 0.342 & 0 & 0 & 0.197 & 2.972 & 3.002 & 2.994 & 2.919 & 0.046 \\
\hline 101.46 & 0 & 0 & 0 & 0 & 0.000 & 2.832 & 2.867 & 2.836 & 2.793 & 0.037 \\
\hline 116.21 & 0 & 0 & 0 & 0 & 0.000 & 2.665 & 2.702 & 2.645 & 2.648 & 0.032 \\
\hline 133.103 & 0 & 0 & 0 & 0 & 0.000 & 2.467 & 2.502 & 2.421 & 2.478 & 0.042 \\
\hline 152.453 & 0 & 0 & 0 & 0 & 0.000 & 2.24 & 2.269 & 2.171 & 2.281 & 0.060 \\
\hline 174.616 & 0 & 0 & 0 & 0 & 0.000 & 1.245 & 1.261 & 1.206 & 1.267 & 0.034 \\
\hline 200 & 0 & 0 & 0 & 0 & 0.000 & 0.691 & 0.7 & 0.67 & 0.704 & 0.019 \\
\hline 229.075 & 0 & 0 & 0 & 0 & 0.000 & 0.384 & 0.389 & 0.372 & 0.391 & 0.010 \\
\hline 262.376 & 0 & 0 & 0 & 0 & 0.000 & 0 & 0 & 0 & 0 & 0.000 \\
\hline 300.518 & 0 & 0 & 0 & 0 & 0.000 & 0 & 0 & 0 & 0 & 0.000 \\
\hline 344.206 & 0 & 0 & 0 & 0 & 0.000 & 0 & 0 & 0 & 0 & 0.000 \\
\hline 394.244 & 0 & 0 & 0 & 0 & 0.000 & 0 & 0 & 0 & 0 & 0.000 \\
\hline 451.556 & 0 & 0 & 0 & 0 & 0.000 & 0 & 0 & 0 & 0 & 0.000 \\
\hline 517.2 & 0 & 0 & 0 & 0 & 0.000 & 0 & 0 & 0 & 0 & 0.000 \\
\hline 592.387 & 0 & 0 & 0 & 0 & 0.000 & 0 & 0 & 0 & 0 & 0.000 \\
\hline
\end{tabular}


Filename

ID\#

:000112-5WM183\#3 s -avg<C>

Circulation Speed :5

Ultra sonic : :00:06

Laser T\% : :78.4(\%)

Form of Distribution:Standard

Calc. Level : :30

R.R.Index $\quad: 1.35-0.10 \mathrm{i}$

Material :WM183 Suspended Solids

Source

Lot Number

Dispersion Medium :RAL demin water

Remarks :GMH/TAB operators

Remarks 1 :19 Jan 2000

Remarks 2 :Avg o Runs 1, 4 \& 5 (w/usonic)

$\begin{array}{lc}\text { Mean } & : 14.761811(\mu \mathrm{m}) \\ \text { Variance } & : 136.578308 \\ \text { S.D. } & : 11.686672(\mu \mathrm{m}) \\ \text { Mode } & : 14.202041(\mu \mathrm{m}) \\ \text { Geo. Mean } & : 10.592593(\mu \mathrm{m})\end{array}$

Filename

ID\#

:000112-5WM183\#3 ns-avg<C>

Circulation Speed :5

Ultra sonic :OFF

Laser T\% : : 80.8(\%)

Form of Distribution:Standard

Calc. Level $\quad: 30$

R.R.Index $\quad: 1.35-0.10 \mathrm{i}$

Material :WM183 Suspended Solids

Source

Lot Number

Dispersion Medium :RAL demin water

Remarks : :GHH/TAB operators

Remarks 1 :19 Jan 2000

Remarks 2 :Avg. 2,3,6 non-sonic

$\begin{array}{lc}\text { Mean } & : 37.176636(\mu \mathrm{m}) \\ \text { Variance } & : 1703.956299 \\ \text { S.D. } & : 41.279007(\mu \mathrm{m}) \\ \text { Mode } & : 14.173473(\mu \mathrm{m}) \\ \text { Geo. Mean } & : 19.812233(\mu \mathrm{m})\end{array}$

\section{WM-183\#3 PSD Analyses}

All samples sonicated; 19 Jan 2000

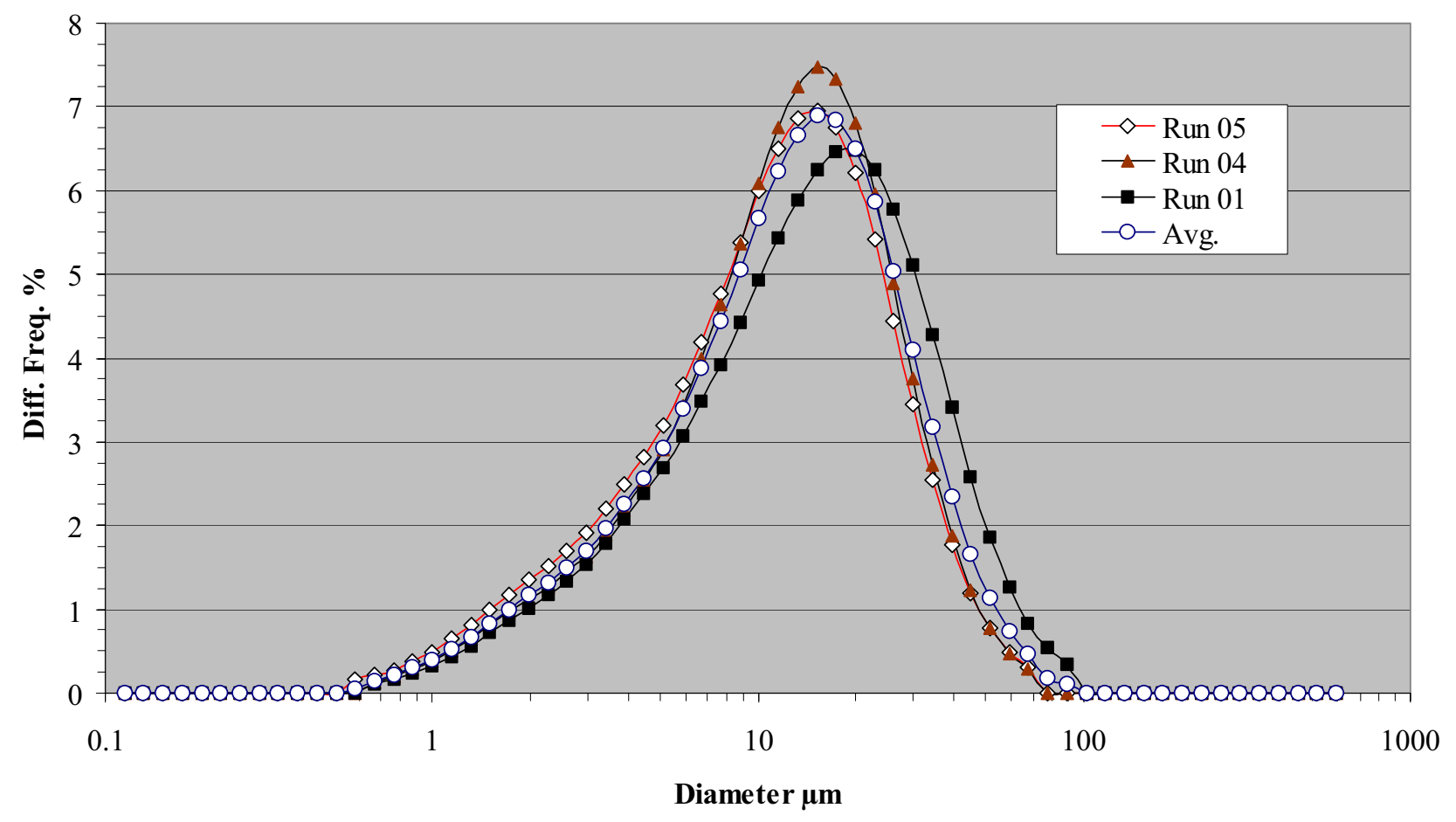




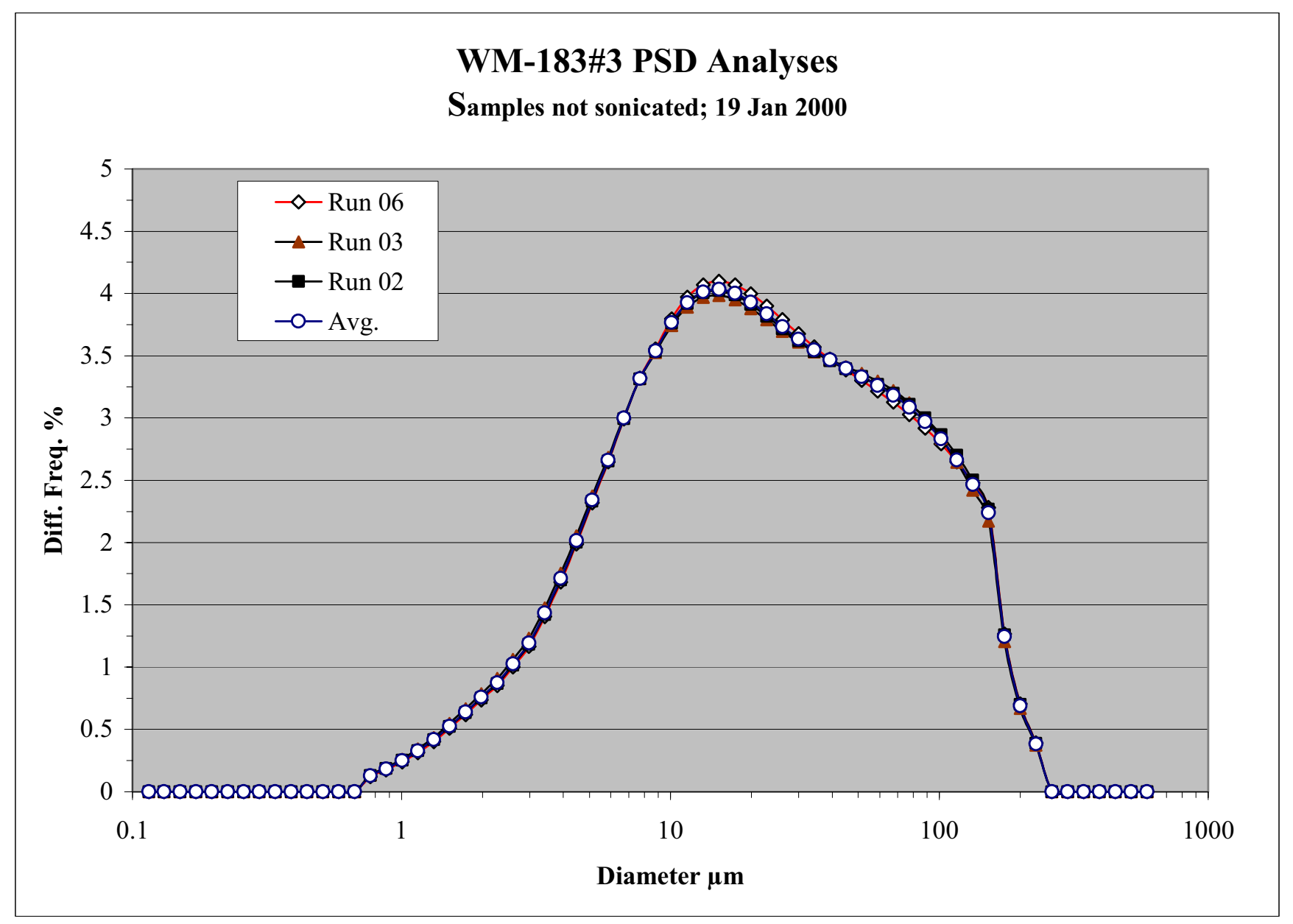


WM183Composite 1,2,3 PSD Analysis

WM183Com 1,2 3 sonicated runs Diff. Vol (\%)

\begin{tabular}{|c|c|c|c|c|}
\hline & & & & \\
\hline Diameter & Avg o Runs & Run 02 & Run 04 & 1 STDEV \\
\hline 0.115 & 0 & 0 & 0 & 0 \\
\hline 0.131 & 0 & 0 & 0 & 0.000 \\
\hline 0.15 & 0 & 0 & 0 & 0.000 \\
\hline 0.172 & 0 & 0 & 0 & 0.000 \\
\hline 0.197 & 0 & 0 & 0 & 0.000 \\
\hline 0.226 & 0 & 0 & 0 & 0.000 \\
\hline 0.259 & 0 & 0 & 0 & 0.000 \\
\hline 0.296 & 0 & 0 & 0 & 0.000 \\
\hline 0.339 & 0 & 0 & 0 & 0.000 \\
\hline 0.389 & 0 & 0 & 0 & 0.000 \\
\hline 0.445 & 0 & 0 & 0 & 0.000 \\
\hline 0.51 & 0 & 0 & 0 & 0.000 \\
\hline 0.584 & 0.134 & 0.119 & 0.149 & 0.021 \\
\hline 0.669 & 0.197 & 0.18 & 0.214 & 0.024 \\
\hline 0.766 & 0.29 & 0.271 & 0.309 & 0.027 \\
\hline 0.877 & 0.417 & 0.397 & 0.438 & 0.029 \\
\hline 1.005 & 0.563 & 0.542 & 0.584 & 0.030 \\
\hline 1.151 & 0.727 & 0.703 & 0.751 & 0.034 \\
\hline 1.318 & 0.911 & 0.884 & 0.939 & 0.039 \\
\hline 1.51 & 1.131 & 1.104 & 1.158 & 0.038 \\
\hline 1.729 & 1.349 & 1.319 & 1.38 & 0.043 \\
\hline 1.981 & 1.582 & 1.547 & 1.617 & 0.049 \\
\hline 2.269 & 1.797 & 1.758 & 1.835 & 0.054 \\
\hline 2.599 & 2.083 & 2.044 & 2.122 & 0.055 \\
\hline 2.976 & 2.404 & 2.365 & 2.444 & 0.056 \\
\hline 3.409 & 2.867 & 2.832 & 2.903 & 0.050 \\
\hline 3.905 & 3.38 & 3.356 & 3.404 & 0.034 \\
\hline 4.472 & 3.911 & 3.906 & 3.917 & 0.008 \\
\hline 5.122 & 4.453 & 4.474 & 4.432 & 0.030 \\
\hline 5.867 & 4.948 & 5.002 & 4.894 & 0.076 \\
\hline 6.72 & 5.449 & 5.538 & 5.361 & 0.125 \\
\hline 7.697 & 5.881 & 6.003 & 5.759 & 0.173 \\
\hline 8.816 & 6.081 & 6.22 & 5.942 & 0.197 \\
\hline 10.097 & 6.259 & 6.412 & 6.106 & 0.216 \\
\hline 11.565 & 6.257 & 6.408 & 6.106 & 0.214 \\
\hline 13.246 & 6.067 & 6.197 & 5.936 & 0.185 \\
\hline 15.172 & 5.697 & 5.792 & 5.602 & 0.134 \\
\hline 17.377 & 5.176 & 5.225 & 5.127 & 0.069 \\
\hline 19.904 & 4.547 & 4.547 & 4.547 & 0.000 \\
\hline 22.797 & 3.859 & 3.816 & 3.903 & 0.062 \\
\hline 26.111 & 3.165 & 3.089 & 3.24 & 0.107 \\
\hline 29.907 & 2.508 & 2.415 & 2.6 & 0.131 \\
\hline 34.255 & 1.921 & 1.826 & 2.017 & 0.135 \\
\hline 39.234 & 1.425 & 1.337 & 1.513 & 0.124 \\
\hline 44.938 & 1.025 & 0.952 & 1.097 & 0.103 \\
\hline 51.471 & 0.717 & 0.662 & 0.772 & 0.078 \\
\hline 58.953 & 0.49 & 0.452 & 0.529 & 0.054 \\
\hline 67.523 & 0.33 & 0.305 & 0.355 & 0.035 \\
\hline 77.339 & 0 & 0 & 0 & 0.000 \\
\hline 88.583 & 0 & 0 & 0 & 0.000 \\
\hline 101.46 & 0 & 0 & 0 & 0.000 \\
\hline 116.21 & 0 & 0 & 0 & 0.000 \\
\hline 133.103 & 0 & 0 & 0 & 0.000 \\
\hline 152.453 & 0 & 0 & 0 & 0.000 \\
\hline 174.616 & 0 & 0 & 0 & 0.000 \\
\hline 200 & 0 & 0 & 0 & 0.000 \\
\hline 229.075 & 0 & 0 & 0 & 0.000 \\
\hline 262.376 & 0 & 0 & 0 & 0.000 \\
\hline 300.518 & 0 & 0 & 0 & 0.000 \\
\hline 344.206 & 0 & 0 & 0 & 0.000 \\
\hline 394.244 & 0 & 0 & 0 & 0.000 \\
\hline 451.556 & 0 & 0 & 0 & 0.000 \\
\hline 517.2 & 0 & 0 & 0 & 0.000 \\
\hline 592.387 & 0 & 0 & 0 & 0.000 \\
\hline
\end{tabular}

WM183Com123 non-sonicated runs Diff. Vol. \%

$\begin{array}{cccc}\text { Avg o Runs } & \text { Run 01 } & \text { Run 03 } & 1 \text { STDEV } \\ 0 & 0 & 0 & 0 \\ 0 & 0 & 0 & 0.000 \\ 0 & 0 & 0 & 0.000 \\ 0 & 0 & 0 & 0.000 \\ 0 & 0 & 0 & 0.000 \\ 0 & 0 & 0 & 0.000 \\ 0 & 0 & 0 & 0.000 \\ 0 & 0 & 0 & 0.000 \\ 0 & 0 & 0 & 0.000 \\ 0 & 0 & 0 & 0.000 \\ 0 & 0 & 0 & 0.000 \\ 0 & 0 & 0 & 0.000 \\ 0.074 & 0.105 & 0.118 & 0.009 \\ 0.11 & 0.156 & 0.173 & 0.012 \\ 0.214 & 0.231 & 0.252 & 0.015 \\ 0.312 & 0.334 & 0.362 & 0.020 \\ 0.426 & 0.453 & 0.487 & 0.024 \\ 0.555 & 0.587 & 0.63 & 0.030 \\ 0.701 & 0.739 & 0.792 & 0.037 \\ 0.871 & 0.925 & 0.987 & 0.044 \\ 1.046 & 1.118 & 1.189 & 0.050 \\ 1.236 & 1.331 & 1.409 & 0.055 \\ 1.419 & 1.535 & 1.618 & 0.059 \\ 1.677 & 1.819 & 1.899 & 0.057 \\ 1.97 & 2.14 & 2.215 & 0.053 \\ 2.414 & 2.614 & 2.672 & 0.041 \\ 2.929 & 3.154 & 3.18 & 0.018 \\ 3.493 & 3.723 & 3.702 & 0.015 \\ 4.089 & 4.298 & 4.222 & 0.054 \\ 4.606 & 4.75 & 4.634 & 0.082 \\ 5.133 & 5.209 & 5.048 & 0.114 \\ 5.559 & 5.557 & 5.361 & 0.139 \\ 5.665 & 5.619 & 5.409 & 0.148 \\ 5.773 & 5.687 & 5.457 & 0.163 \\ 5.696 & 5.599 & 5.353 & 0.174 \\ 5.455 & 5.377 & 5.117 & 0.184 \\ 5.09 & 5.051 & 4.778 & 0.193 \\ 4.651 & 4.66 & 4.372 & 0.204 \\ 4.18 & 4.234 & 3.934 & 0.212 \\ 3.715 & 3.799 & 3.494 & 0.216 \\ 3.276 & 3.373 & 3.074 & 0.211 \\ 2.877 & 2.965 & 2.689 & 0.195 \\ 2.519 & 2.581 & 2.344 & 0.168 \\ 2.199 & 2.22 & 2.041 & 0.127 \\ 1.912 & 1.882 & 1.779 & 0.073 \\ 1.652 & 1.569 & 1.553 & 0.011 \\ 1.415 & 1.284 & 1.361 & 0.054 \\ 1.201 & 1.03 & 1.198 & 0.119 \\ 1.01 & 0.811 & 1.06 & 0.176 \\ 0.841 & 0.629 & 0.943 & 0.222 \\ 0.694 & 0.483 & 0.839 & 0.252 \\ 0.569 & 0.369 & 0.742 & 0.264 \\ 0.369 & 0 & 0.648 & 0.458 \\ 0.303 & 0 & 0.556 & 0.393 \\ 0.103 & 0 & 0.309 & 0.218 \\ 0 & 0 & 0 & 0.000 \\ 0 & 0 & 0 & 0.000 \\ 0 & 0 & 0 & 0.000 \\ 0 & 0 & 0 & 0.000 \\ 0 & 0 & 0 & 0.000 \\ 0 & 0 & 0 & 0.000 \\ 0 & 0 & 0 & 0.000 \\ 0 & 0 & 0 & 0.000 \\ 0 & 0 & 0 & 0.000 \\ & & & \\ 0 & 0 & 0 & \end{array}$




\begin{tabular}{|c|c|c|c|}
\hline $\begin{array}{l}\text { Filename } \\
\text { ID\# }\end{array}$ & $\begin{array}{l}: 000105-6 \text { WM183-1,2,3,com s-avg<C> } \\
: 200001191515075\end{array}$ & $\begin{array}{l}\text { Filename } \\
\text { ID\# }\end{array}$ & $\begin{array}{l}: 000105-6 \text { ns WM183-avg<C> } \\
: 200003081011101\end{array}$ \\
\hline \multicolumn{2}{|c|}{ Circulation Speed $: 5$} & \multicolumn{2}{|c|}{ Circulation Speed $: 6$} \\
\hline Ultra sonic & $: 00: 24$ & Ultra sonic & :OFF \\
\hline Laser T\% & : 90.3(\%) & Laser T\% & : 92.0(\%) \\
\hline \multicolumn{2}{|c|}{ Form of Distribution:Standard } & \multicolumn{2}{|c|}{ Form of Distribution:Standard } \\
\hline Calc. Level & :30 & Calc. Level & :30 \\
\hline R.R.Index & $: 1.35-0.10 \mathrm{i}$ & R.R.Index & :1.35-0.10i \\
\hline Material & :WM183 Solids Composite & Material & :WM183Compos1,2,3 \\
\hline Source & $:$ & Source & $:$ \\
\hline Lot Number & : & Lot Number & : \\
\hline \multicolumn{2}{|c|}{ Dispersion Medium :RAL demin water + usonic } & \multicolumn{2}{|c|}{ Dispersion Medium :RAL demin water } \\
\hline Remarks & :GHH/TAB operators & Remarks & :GMH operator \\
\hline Remarks 1 & :19 Jan 2000 & Remarks 1 & :8 Mar 2000 \\
\hline Remarks 2 & :Avg Runs 2, 4 & Remarks 2 & : \\
\hline Mean & $: 11.579919(\mu \mathrm{m})$ & Mean & $: 17.159410(\mu \mathrm{m})$ \\
\hline Variance & :100.125282 & Variance & :438.032654 \\
\hline S.D. & $: 10.006262(\mu \mathrm{m})$ & S.D. & $: 20.929230(\mu \mathrm{m})$ \\
\hline Mode & : 9.452659( $\mu \mathrm{m})$ & Mode & : 9.438090( $\mu \mathrm{m})$ \\
\hline Geo. Mean & $: 8.138731(\mu \mathrm{m})$ & Geo. Mean & $: 10.284929(\mu \mathrm{m})$ \\
\hline
\end{tabular}

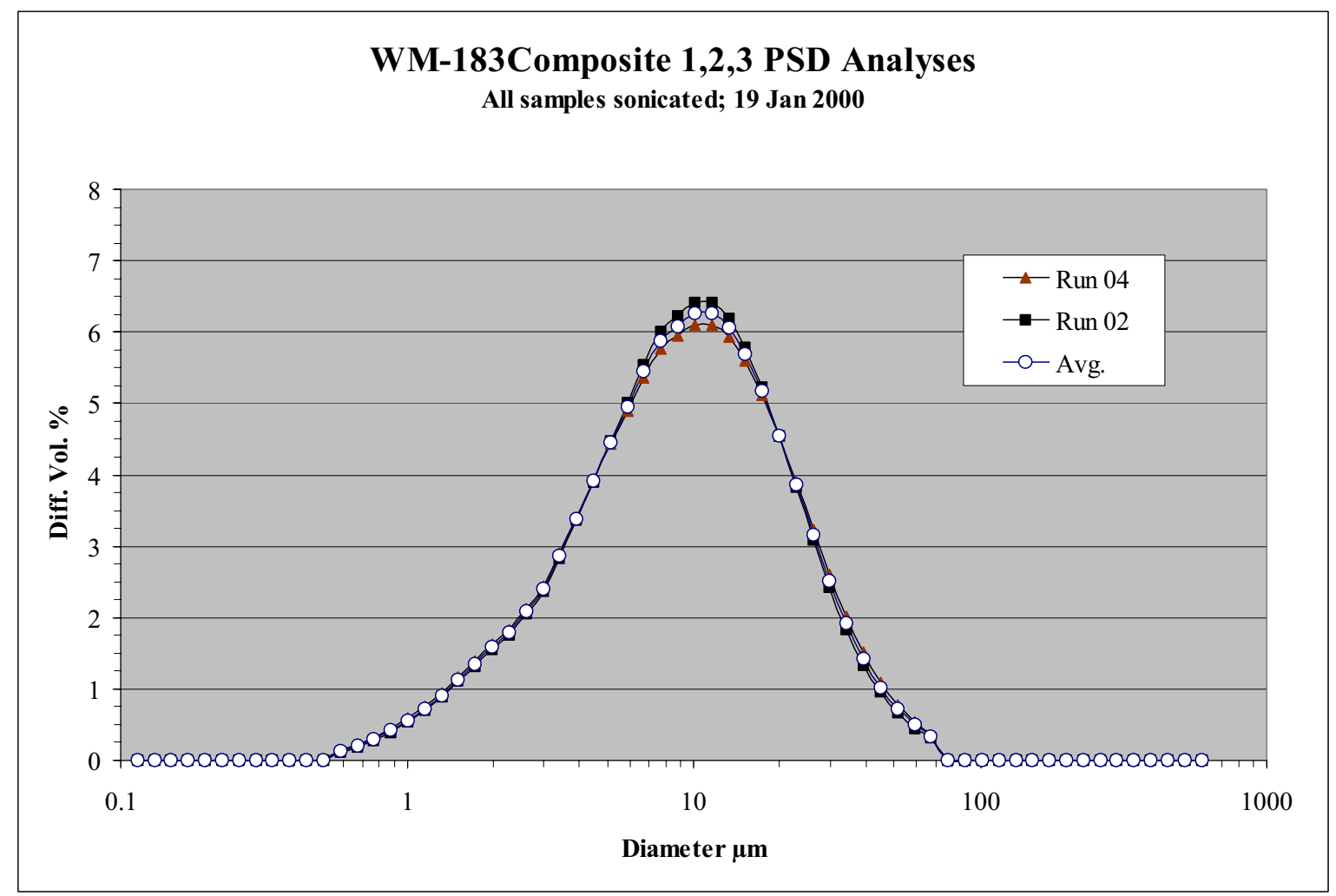




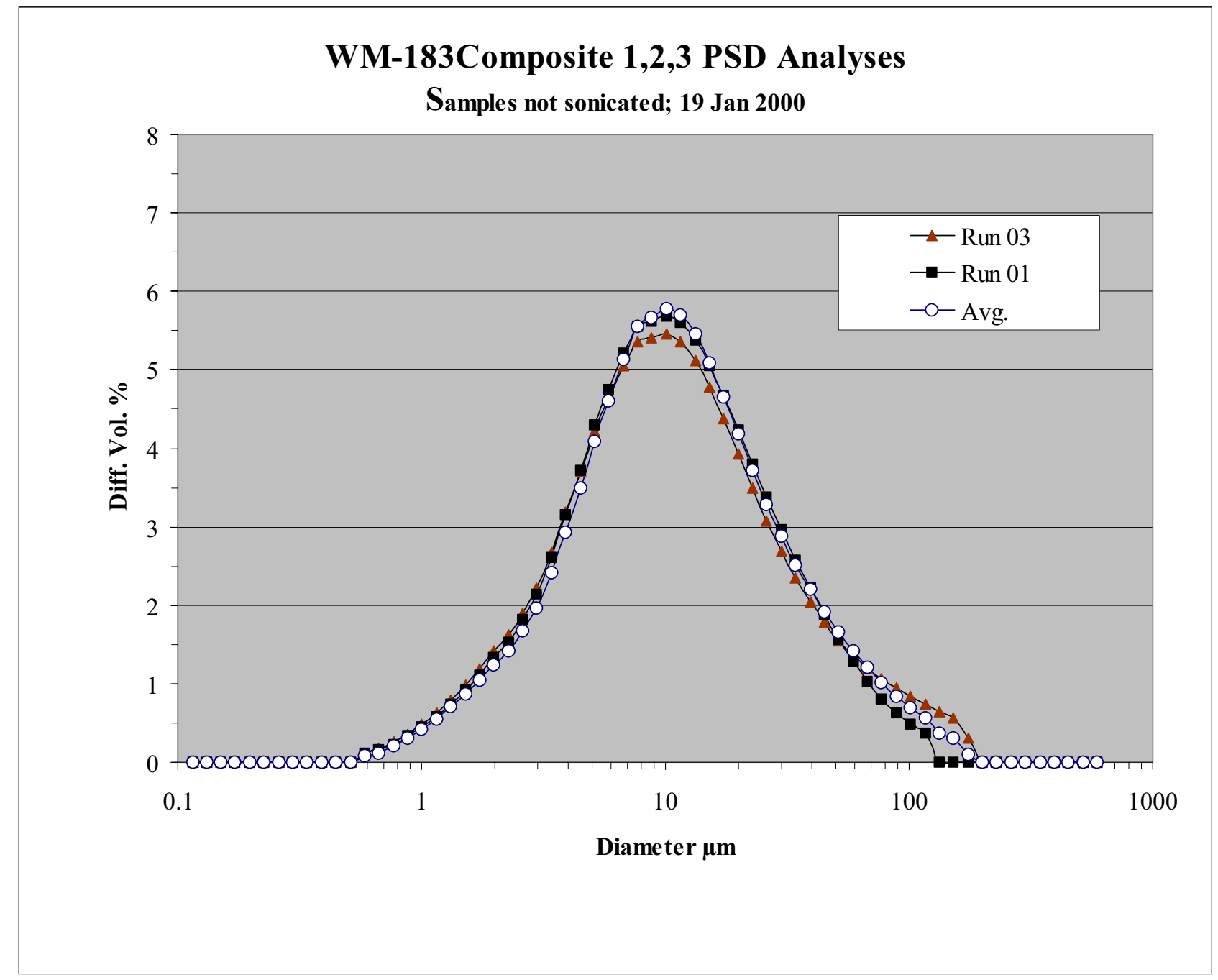


WM183 Composite B PSD Analysis

sonicated runs

Diff. Frequency (\%)

\begin{tabular}{|c|c|c|c|c|c|c|}
\hline Diameter & Avg o Runs & Run 01 & Run 03 & Run 05 & Run 06 & 1 STDEV \\
\hline 0.115 & 0 & 0 & 0 & 0 & 0 & 0 \\
\hline 0.131 & 0 & 0 & 0 & 0 & 0 & 0.000 \\
\hline 0.15 & 0 & 0 & 0 & 0 & 0 & 0.000 \\
\hline 0.172 & 0 & 0 & 0 & 0 & 0 & 0.000 \\
\hline 0.197 & 0 & 0 & 0 & 0 & 0 & 0.000 \\
\hline 0.226 & 0 & 0 & 0 & 0 & 0 & 0.000 \\
\hline 0.259 & 0 & 0 & 0 & 0 & 0 & 0.000 \\
\hline 0.296 & 0 & 0 & 0 & 0 & 0 & 0.000 \\
\hline 0.339 & 0 & 0 & 0 & 0 & 0 & 0.000 \\
\hline 0.389 & 0 & 0 & 0 & 0 & 0 & 0.000 \\
\hline 0.445 & 0 & 0 & 0 & 0 & 0 & 0.000 \\
\hline 0.51 & 0 & 0 & 0 & 0 & 0 & 0.000 \\
\hline 0.584 & 0.125 & 0.121 & 0.111 & 0.12 & 0.143 & 0.014 \\
\hline 0.669 & 0.176 & 0.163 & 0.159 & 0.169 & 0.199 & 0.018 \\
\hline 0.766 & 0.248 & 0.223 & 0.227 & 0.241 & 0.278 & 0.025 \\
\hline 0.877 & 0.347 & 0.306 & 0.32 & 0.338 & 0.384 & 0.034 \\
\hline 1.005 & 0.461 & 0.404 & 0.429 & 0.451 & 0.505 & 0.043 \\
\hline 1.151 & 0.592 & 0.521 & 0.554 & 0.579 & 0.642 & 0.051 \\
\hline 1.318 & 0.735 & 0.654 & 0.692 & 0.72 & 0.792 & 0.058 \\
\hline 1.51 & 0.898 & 0.801 & 0.853 & 0.882 & 0.959 & 0.066 \\
\hline 1.729 & 1.054 & 0.952 & 1.006 & 1.036 & 1.119 & 0.070 \\
\hline 1.981 & 1.212 & 1.111 & 1.161 & 1.191 & 1.283 & 0.072 \\
\hline 2.269 & 1.354 & 1.258 & 1.303 & 1.331 & 1.429 & 0.072 \\
\hline 2.599 & 1.531 & 1.437 & 1.474 & 1.503 & 1.615 & 0.077 \\
\hline 2.976 & 1.721 & 1.633 & 1.66 & 1.689 & 1.816 & 0.081 \\
\hline 3.409 & 1.998 & 1.904 & 1.923 & 1.956 & 2.115 & 0.096 \\
\hline 3.905 & 2.311 & 2.197 & 2.214 & 2.258 & 2.46 & 0.121 \\
\hline 4.472 & 2.663 & 2.504 & 2.534 & 2.599 & 2.857 & 0.161 \\
\hline 5.122 & 3.076 & 2.832 & 2.898 & 3 & 3.33 & 0.221 \\
\hline 5.867 & 3.635 & 3.203 & 3.379 & 3.55 & 3.975 & 0.331 \\
\hline 6.72 & 4.235 & 3.599 & 3.899 & 4.143 & 4.664 & 0.451 \\
\hline 7.697 & 4.92 & 4.019 & 4.493 & 4.826 & 5.44 & 0.597 \\
\hline 8.816 & 5.627 & 4.448 & 5.148 & 5.553 & 6.18 & 0.726 \\
\hline 10.097 & 6.33 & 4.889 & 5.815 & 6.283 & 6.893 & 0.845 \\
\hline 11.565 & 6.924 & 5.298 & 6.433 & 6.918 & 7.421 & 0.908 \\
\hline 13.246 & 7.304 & 5.644 & 6.917 & 7.352 & 7.642 & 0.882 \\
\hline 15.172 & 7.372 & 5.892 & 7.175 & 7.475 & 7.465 & 0.753 \\
\hline 17.377 & 7.072 & 6.007 & 7.126 & 7.218 & 6.873 & 0.552 \\
\hline 19.904 & 6.415 & 5.956 & 6.729 & 6.577 & 5.939 & 0.412 \\
\hline 22.797 & 5.483 & 5.721 & 6.008 & 5.634 & 4.807 & 0.516 \\
\hline 26.111 & 4.412 & 5.305 & 5.056 & 4.529 & 3.651 & 0.732 \\
\hline 29.907 & 3.346 & 4.737 & 4.004 & 3.422 & 2.613 & 0.899 \\
\hline 34.255 & 2.399 & 4.064 & 2.986 & 2.438 & 1.773 & 0.969 \\
\hline 39.234 & 1.633 & 3.347 & 2.104 & 1.647 & 1.15 & 0.941 \\
\hline 44.938 & 1.064 & 2.647 & 1.408 & 1.063 & 0.72 & 0.840 \\
\hline 51.471 & 0.669 & 2.015 & 0.902 & 0.662 & 0.441 & 0.699 \\
\hline 58.953 & 0.41 & 1.484 & 0.56 & 0.403 & 0.267 & 0.550 \\
\hline 67.523 & 0.249 & 1.064 & 0.341 & 0.243 & 0.163 & 0.414 \\
\hline 77.339 & 0 & 0.749 & 0 & 0 & 0 & 0.375 \\
\hline 88.583 & 0 & 0.524 & 0 & 0 & 0 & 0.262 \\
\hline 101.46 & 0 & 0.368 & 0 & 0 & 0 & 0.184 \\
\hline 116.21 & 0 & 0 & 0 & 0 & 0 & 0.000 \\
\hline 133.103 & 0 & 0 & 0 & 0 & 0 & 0.000 \\
\hline 152.453 & 0 & 0 & 0 & 0 & 0 & 0.000 \\
\hline 174.616 & 0 & 0 & 0 & 0 & 0 & 0.000 \\
\hline 200 & 0 & 0 & 0 & 0 & 0 & 0.000 \\
\hline 229.075 & 0 & 0 & 0 & 0 & 0 & 0.000 \\
\hline 262.376 & 0 & 0 & 0 & 0 & 0 & 0.000 \\
\hline 300.518 & 0 & 0 & 0 & 0 & 0 & 0.000 \\
\hline 344.206 & 0 & 0 & 0 & 0 & 0 & 0.000 \\
\hline 394.244 & 0 & 0 & 0 & 0 & 0 & 0.000 \\
\hline 451.556 & 0 & 0 & 0 & 0 & 0 & 0.000 \\
\hline 517.2 & 0 & 0 & 0 & 0 & 0 & 0.000 \\
\hline 592.387 & 0 & 0 & 0 & 0 & 0 & 0.000 \\
\hline
\end{tabular}

on-sonicated runs

Diff. Frequency \%

Avgo Runs Run 02 Run 04 STDEV

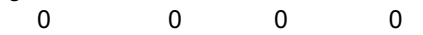

$\begin{array}{cccc}0 & 0 & 0 & 0 \\ 0 & 0 & 0 & 0.000\end{array}$

$\begin{array}{llll}0 & 0 & 0 & 0.000 \\ 0 & 0 & 0 & 0.000\end{array}$

$\begin{array}{llll}0 & 0 & 0 & 0.000 \\ 0 & 0 & 0 & 0.000\end{array}$

$\begin{array}{llll}0 & 0 & 0 & 0.000\end{array}$

$\begin{array}{llll}0 & 0 & 0 & 0.000 \\ 0 & 0 & 0 & 0.000\end{array}$

$\begin{array}{llll}0 & 0 & 0 & 0.000 \\ 0 & 0 & 0 & 0.000\end{array}$

$\begin{array}{llll}0 & 0 & 0 & 0.000\end{array}$

$\begin{array}{llll}0 & 0 & 0 & 0.000 \\ 0 & 0 & 0 & 0.000\end{array}$

$\begin{array}{cccc}0 & 0 & 0 & 0.000 \\ 0.058 & 0.117 & 0 & 0.083\end{array}$

$\begin{array}{llll}0.152 & 0.162 & 0.142 & 0.014\end{array}$

$\begin{array}{llll}0.212 & 0.224 & 0.2 & 0.017\end{array}$

$\begin{array}{llll}0.283 & 0.297 & 0.269 & 0.020\end{array}$

$\begin{array}{llll}0.365 & 0.383 & 0.348 & 0.025\end{array}$

$\begin{array}{llll}0.459 & 0.481 & 0.438 & 0.030\end{array}$

$\begin{array}{llll}0.568 & 0.591 & 0.545 & 0.033\end{array}$

$\begin{array}{llll}0.68 & 0.707 & 0.654 & 0.037\end{array}$

$\begin{array}{llll}0.801 & 0.831 & 0.772 & 0.042\end{array}$

$\begin{array}{llll}0.917 & 0.949 & 0.884 & 0.046\end{array}$

$\begin{array}{llll}1.067 & 1.102 & 1.032 & 0.049\end{array}$

$\begin{array}{llll}1.231 & 1.269 & 1.194 & 0.053\end{array}$

$\begin{array}{llll}1.471 & 1.511 & 1.431 & 0.057\end{array}$

$\begin{array}{llll}1.744 & 1.784 & 1.703 & 0.057\end{array}$

$\begin{array}{llll}2.04 & 2.078 & 2.002 & 0.054\end{array}$

$\begin{array}{llll}2.358 & 2.392 & 2.324 & 0.048\end{array}$

$\begin{array}{llll}2.677 & 2.706 & 2.648 & 0.041\end{array}$

$\begin{array}{llll}3.009 & 3.031 & 2.986 & 0.032\end{array}$

$\begin{array}{llll}3.323 & 3.339 & 3.308 & 0.022\end{array}$

$\begin{array}{llll}3.546 & 3.557 & 3.536 & 0.015\end{array}$

$\begin{array}{llll}3.775 & 3.779 & 3.771 & 0.006\end{array}$

$\begin{array}{llll}3.935 & 3.935 & 3.936 & 0.001\end{array}$

$\begin{array}{llll}4.024 & 4.02 & 4.029 & 0.006\end{array}$

$\begin{array}{llll}4.046 & 4.038 & 4.054 & 0.011\end{array}$

$\begin{array}{llll}4.014 & 4.003 & 4.024 & 0.015\end{array}$

$\begin{array}{llll}3.942 & 3.928 & 3.955 & 0.019\end{array}$

$\begin{array}{llll}3.848 & 3.831 & 3.865 & 0.024\end{array}$

$\begin{array}{llll}3.746 & 3.725 & 3.767 & 0.030\end{array}$

$\begin{array}{llll}3.65 & 3.623 & 3.676 & 0.037\end{array}$

$\begin{array}{llll}3.563 & 3.531 & 3.595 & 0.045\end{array}$

$\begin{array}{llll}3.488 & 3.449 & 3.527 & 0.055\end{array}$

$\begin{array}{llll}3.42 & 3.374 & 3.465 & 0.064\end{array}$

$\begin{array}{llll}3.351 & 3.3 & 3.403 & 0.073\end{array}$

$\begin{array}{llll}3.275 & 3.219 & 3.331 & 0.079\end{array}$

$\begin{array}{llll}3.181 & 3.124 & 3.239 & 0.081\end{array}$

$\begin{array}{llll}3.064 & 3.009 & 3.118 & 0.077\end{array}$

$\begin{array}{llll}2.918 & 2.871 & 2.966 & 0.067\end{array}$

$\begin{array}{llll}2.744 & 2.708 & 2.781 & 0.052\end{array}$

$\begin{array}{llll}2.542 & 2.518 & 2.565 & 0.033\end{array}$

$\begin{array}{llll}2.312 & 2.302 & 2.322 & 0.014\end{array}$

$\begin{array}{llll}2.062 & 2.064 & 2.061 & 0.002\end{array}$

$\begin{array}{llll}1.146 & 1.147 & 1.145 & 0.001\end{array}$

$\begin{array}{llll}0.637 & 0.637 & 0.636 & 0.001\end{array}$

$\begin{array}{llll}0.354 & 0.354 & 0.353 & 0.001\end{array}$

$\begin{array}{cccc}0 & 0 & 0 & 0.000\end{array}$

$\begin{array}{llll}0 & 0 & 0 & 0.000 \\ 0 & 0 & 0 & 0.000\end{array}$

$0=0.000$

$\begin{array}{llll}0 & 0 & 0 & 0.000 \\ 0 & 0 & 0 & 0.000\end{array}$

$\begin{array}{llll}0 & 0 & 0 & 0.000 \\ 0 & 0 & 0 & 0.000\end{array}$ 


\begin{tabular}{|c|c|}
\hline Filename & :000117-5WM183ComB s avg<C> \\
\hline ID\# & $: 200002071150080$ \\
\hline \multicolumn{2}{|c|}{ Circulation Speed $: 5$} \\
\hline Ultra sonic & :01:15 \\
\hline Laser T\% & $: 72.1(\%)$ \\
\hline \multicolumn{2}{|c|}{ Form of Distribution:Standard } \\
\hline Calc. Level & :30 \\
\hline R.R.Index & $: 1.35-0.10 \mathrm{i}$ \\
\hline Material & :WM183 Solids Composite B \\
\hline Source & $:$ \\
\hline Lot Number & : \\
\hline \multicolumn{2}{|c|}{ Dispersion Medium $\quad$ RAL demin water + usonic } \\
\hline Remarks & :GHH/TAB operators \\
\hline Remarks 1 & :07 Feb 2000 \\
\hline Remarks 2 & :Avg. Runs 3,5,6 \\
\hline Mean & $: 13.214108(\mu \mathrm{m})$ \\
\hline Variance & :97.520866 \\
\hline S.D. & $: 9.875265(\mu \mathrm{m})$ \\
\hline Mode & $: 14.146187(\mu \mathrm{m})$ \\
\hline Geo. Mean & : 9.731933( $\mu \mathrm{m})$ \\
\hline
\end{tabular}

\begin{tabular}{|c|c|}
\hline Filename & :000117-5WM183ComB ns avg<C> \\
\hline ID\# & :200002071130079 \\
\hline \multicolumn{2}{|c|}{ Circulation Speed :5 } \\
\hline Ultra sonic & :OFF \\
\hline Laser T\% & $: 68.2(\%)$ \\
\hline \multicolumn{2}{|c|}{ Form of Distribution:Standard } \\
\hline Calc. Level & $: 30$ \\
\hline R.R.Index & $: 1.35-0.10 \mathrm{i}$ \\
\hline Material & :WM183 Solids Composite B \\
\hline Source & $:$ \\
\hline Lot Number & : \\
\hline \multicolumn{2}{|c|}{ Dispersion Medium :RAL demin water + usonic } \\
\hline Remarks & :GHH/TAB operators \\
\hline Remarks 1 & :07 Feb 2000 \\
\hline Remarks 2 & :Avg Runs 2,4 \\
\hline Mean & $: 36.193790(\mu \mathrm{m})$ \\
\hline Variance & :1631.289551 \\
\hline S.D. & $: 40.389225(\mu \mathrm{m})$ \\
\hline Mode & $: 14.173810(\mu \mathrm{m})$ \\
\hline Geo. Mean & $: 19.242262(\mu \mathrm{m})$ \\
\hline
\end{tabular}

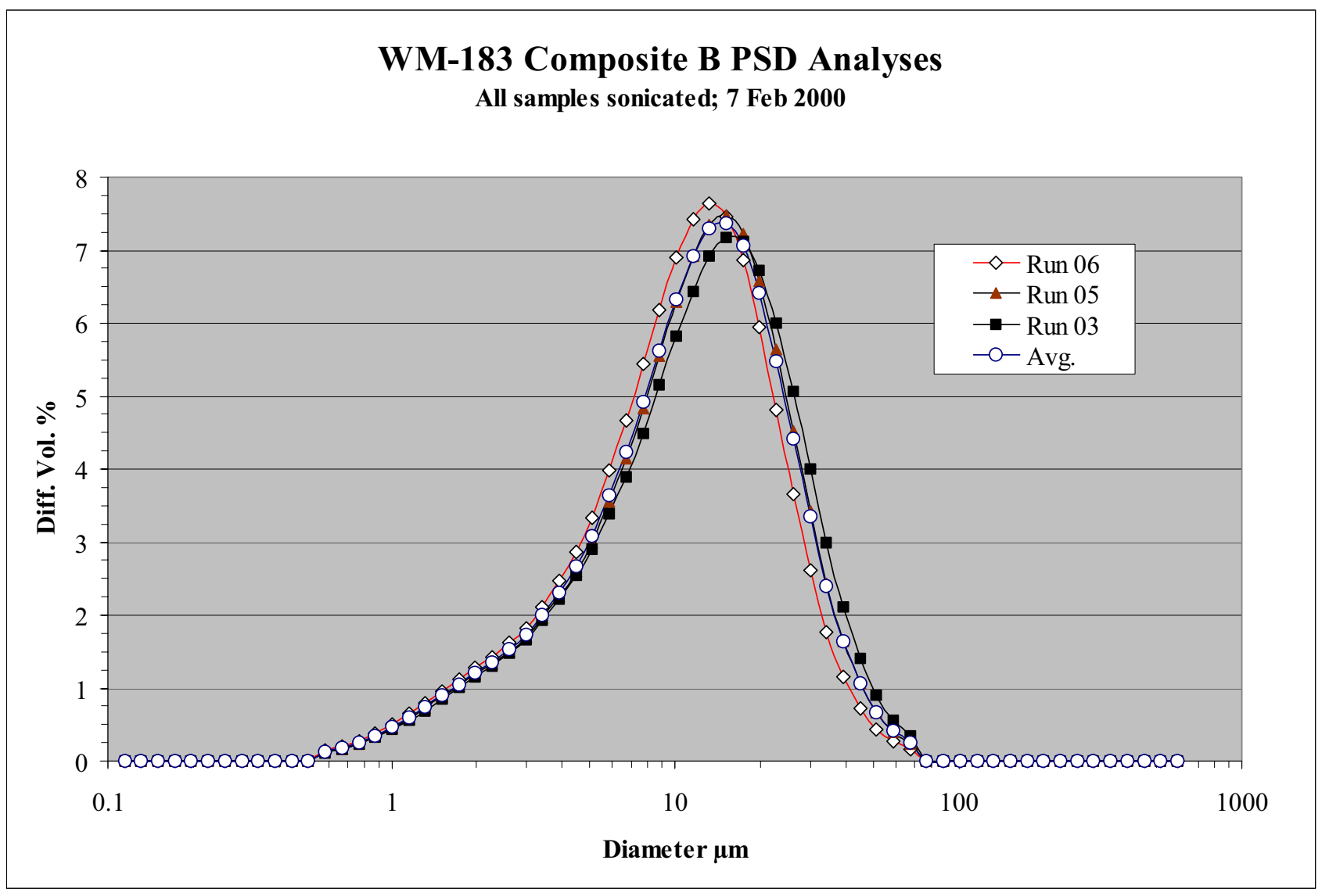




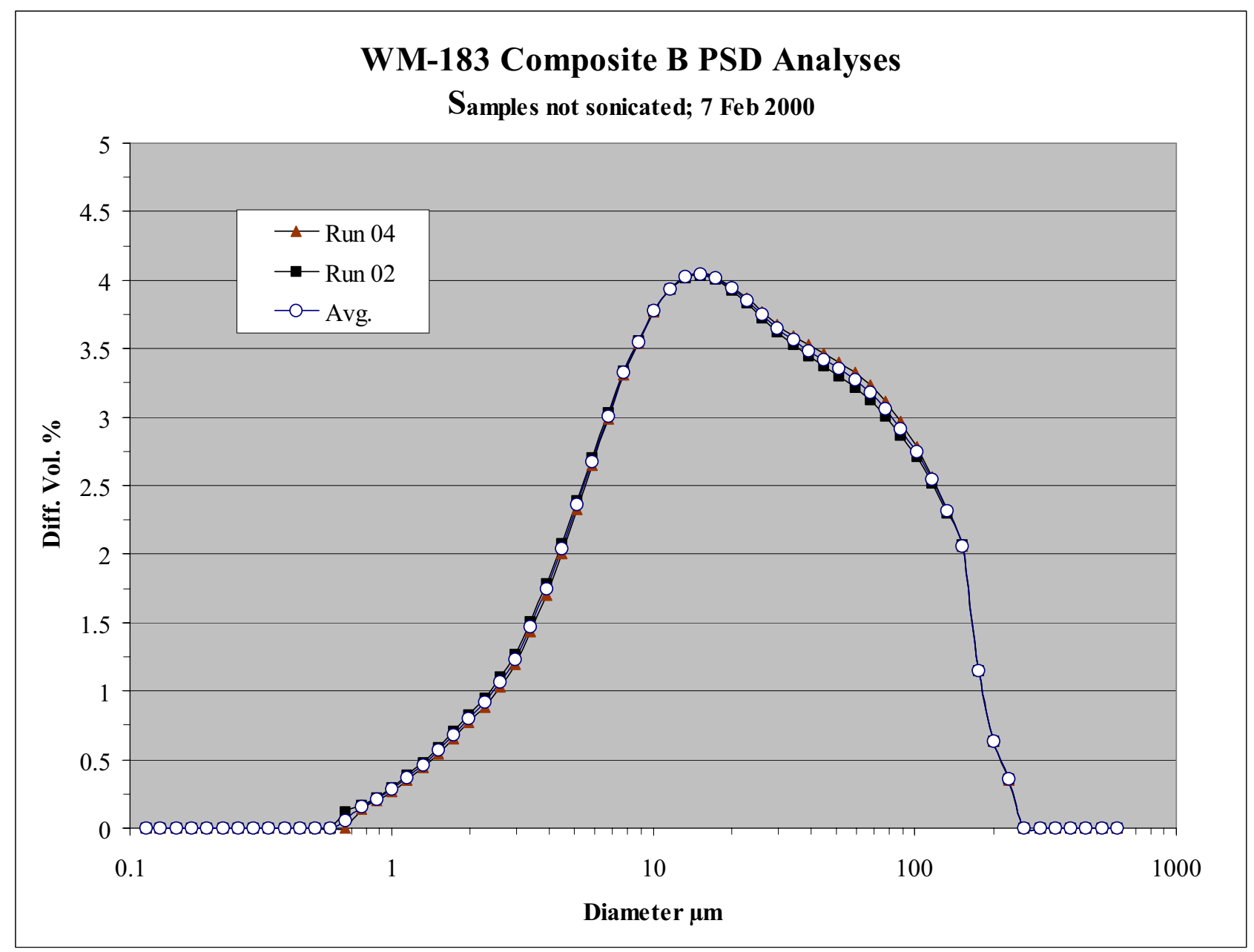


WM182 Settling Rate PSD vs. Time Testing Data

\begin{tabular}{|c|c|c|c|c|c|c|c|c|c|}
\hline Hori & D Ana & Diff. Fre & ; @ R & 8 Feb 2 & ; all sa & es sor & & & \\
\hline $\begin{array}{c}\text { Diameter } \\
{[\mu \mathrm{m}]}\end{array}$ & & & & Fre & ency $(\%)$ & & & & \\
\hline Time [min] & 0 & 1 & 19 & 33 & 47 & 60 & 90 & 160 & 300 \\
\hline 0.115 & 0 & 0 & 0 & 0 & 0 & 0 & 0 & 0 & 0 \\
\hline 0.131 & 0 & 0 & 0 & 0 & 0 & 0 & 0 & 0 & 0 \\
\hline 0.15 & 0 & 0 & 0 & 0 & 0 & 0 & 0 & 0 & 0 \\
\hline 0.172 & 0 & 0 & 0 & 0 & 0 & 0 & 0 & 0 & 0 \\
\hline 0.197 & 0 & 0 & 0 & 0 & 0 & 0 & 0 & 0 & 0 \\
\hline 0.226 & 0 & 0 & 0 & 0 & 0 & 0 & 0 & 0 & 0 \\
\hline 0.259 & 0 & 0 & 0 & 0 & 0 & 0 & 0 & 0 & 0 \\
\hline 0.296 & 0 & 0 & 0 & 0 & 0 & 0 & 0 & 0 & 0 \\
\hline 0.339 & 0 & 0 & 0 & 0 & 0 & 0 & 0 & 0 & 0 \\
\hline 0.389 & 0 & 0 & 0 & 0 & 0 & 0 & 0 & 0 & 0 \\
\hline 0.445 & 0.114 & 0 & 0 & 0 & 0 & 0 & 0 & 0 & 0 \\
\hline 0.51 & 0.14 & 0 & 0 & 0 & 0 & 0 & 0 & 0 & 0 \\
\hline 0.584 & 0.342 & 0.303 & 0.276 & 0.336 & 0.37 & 0.343 & 0.378 & 0.376 & 0.241 \\
\hline 0.669 & 0.462 & 0.415 & 0.381 & 0.475 & 0.525 & 0.484 & 0.537 & 0.547 & 0.349 \\
\hline 0.766 & 0.612 & 0.555 & 0.512 & 0.653 & 0.724 & 0.667 & 0.742 & 0.775 & 0.492 \\
\hline 0.877 & 0.787 & 0.718 & 0.664 & 0.863 & 0.96 & 0.885 & 0.988 & 1.054 & 0.669 \\
\hline 1.005 & 0.949 & 0.864 & 0.799 & 1.052 & 1.174 & 1.083 & 1.211 & 1.315 & 0.836 \\
\hline 1.151 & 1.121 & 1.007 & 0.93 & 1.237 & 1.384 & 1.279 & 1.432 & 1.573 & 1.006 \\
\hline 1.318 & 1.297 & 1.155 & 1.068 & 1.435 & 1.606 & 1.49 & 1.667 & 1.848 & 1.19 \\
\hline 1.51 & 1.469 & 1.317 & 1.222 & 1.658 & 1.854 & 1.727 & 1.929 & 2.16 & 1.403 \\
\hline 1.729 & 1.664 & 1.49 & 1.395 & 1.911 & 2.133 & 1.996 & 2.223 & 2.499 & 1.635 \\
\hline 1.981 & 1.877 & 1.683 & 1.593 & 2.203 & 2.458 & 2.309 & 2.562 & 2.885 & 1.894 \\
\hline 2.269 & 2.059 & 1.847 & 1.767 & 2.463 & 2.745 & 2.588 & 2.861 & 3.226 & 2.125 \\
\hline 2.599 & 2.336 & 2.102 & 2.044 & 2.882 & 3.219 & 3.038 & 3.344 & 3.77 & 2.462 \\
\hline 2.976 & 2.66 & 2.392 & 2.364 & 3.37 & 3.771 & 3.565 & 3.91 & 4.411 & 2.863 \\
\hline 3.409 & 3.126 & 2.816 & 2.84 & 4.09 & 4.6 & 4.337 & 4.742 & 5.336 & 3.394 \\
\hline 3.905 & 3.618 & 3.276 & 3.364 & 4.873 & 5.509 & 5.163 & 5.634 & 6.3 & 3.901 \\
\hline 4.472 & 4.094 & 3.734 & 3.886 & 5.616 & 6.388 & 5.923 & 6.464 & 7.15 & 4.273 \\
\hline 5.122 & 4.556 & 4.192 & 4.39 & 6.264 & 7.165 & 6.542 & 7.16 & 7.79 & 4.462 \\
\hline 5.867 & 4.964 & 4.628 & 4.763 & 6.525 & 7.465 & 6.641 & 7.342 & 7.747 & 4.194 \\
\hline 6.72 & 5.386 & 5.083 & 5.141 & 6.769 & 7.716 & 6.715 & 7.487 & 7.673 & 3.961 \\
\hline 7.697 & 5.754 & 5.507 & 5.408 & 6.761 & 7.619 & 6.484 & 7.299 & 7.219 & 3.566 \\
\hline 8.816 & 5.964 & 5.814 & 5.408 & 6.26 & 6.785 & 5.694 & 6.455 & 6.072 & 2.936 \\
\hline 10.097 & 6.139 & 6.09 & 5.396 & 5.819 & 6.036 & 5.036 & 5.719 & 5.126 & 2.458 \\
\hline 11.565 & 6.152 & 6.219 & 5.224 & 5.212 & 5.071 & 4.266 & 4.813 & 4.085 & 1.998 \\
\hline 13.246 & 5.977 & 6.165 & 4.91 & 4.513 & 4.02 & 3.482 & 3.852 & 3.078 & 1.596 \\
\hline 15.172 & 5.603 & 5.903 & 4.488 & 3.793 & 3.008 & 2.761 & 2.939 & 2.198 & 1.269 \\
\hline 17.377 & 5.041 & 5.433 & 4.004 & 3.109 & 2.13 & 2.147 & 2.144 & 1.494 & 1.021 \\
\hline 19.904 & 4.327 & 4.783 & 3.5 & 2.493 & 1.432 & 1.656 & 1.503 & 0.971 & 0.842 \\
\hline 22.797 & 3.525 & 4.015 & 3.014 & 1.963 & 0.918 & 1.282 & 1.016 & 0.607 & 0.724 \\
\hline 26.111 & 2.713 & 3.206 & 2.576 & 1.522 & 0.566 & 1.009 & 0.668 & 0.368 & 0.658 \\
\hline 29.907 & 1.966 & 2.436 & 2.201 & 1.162 & 0.338 & 0.816 & 0.429 & 0.218 & 0.638 \\
\hline 34.255 & 1.34 & 1.766 & 1.894 & 0.874 & 0.197 & 0.686 & 0.272 & 0.128 & 0.667 \\
\hline 39.234 & 0.859 & 1.224 & 1.653 & 0.646 & 0.114 & 0.604 & 0.171 & 0 & 0.753 \\
\hline 44.938 & 0.521 & 0.815 & 1.469 & 0.469 & 0 & 0.558 & 0.107 & 0 & 0.912 \\
\hline 51.471 & 0.301 & 0.523 & 1.332 & 0.334 & 0 & 0.542 & 0 & 0 & 1.175 \\
\hline 58.953 & 0.135 & 0.326 & 1.232 & 0.234 & 0 & 0.551 & 0 & 0 & 1.578 \\
\hline 67.523 & 0.049 & 0.199 & 1.157 & 0.161 & 0 & 0.582 & 0 & 0 & 2.156 \\
\hline 77.339 & 0 & 0 & 1.095 & 0 & 0 & 0.633 & 0 & 0 & 2.915 \\
\hline 88.583 & 0 & 0 & 1.034 & 0 & 0 & 0.698 & 0 & 0 & 3.774 \\
\hline 101.46 & 0 & 0 & 0.962 & 0 & 0 & 0.768 & 0 & 0 & 4.542 \\
\hline 116.21 & 0 & 0 & 0.873 & 0 & 0 & 0.826 & 0 & 0 & 4.954 \\
\hline 133.103 & 0 & 0 & 0.765 & 0 & 0 & 0.852 & 0 & 0 & 4.815 \\
\hline 152.453 & 0 & 0 & 0.649 & 0 & 0 & 0.83 & 0 & 0 & 4.155 \\
\hline 174.616 & 0 & 0 & 0.36 & 0 & 0 & 0.461 & 0 & 0 & 3.215 \\
\hline 200 & 0 & 0 & 0 & 0 & 0 & 0 & 0 & 0 & 2.281 \\
\hline 229.075 & 0 & 0 & 0 & 0 & 0 & 0 & 0 & 0 & 1.522 \\
\hline 262.376 & 0 & 0 & 0 & 0 & 0 & 0 & 0 & 0 & 0.983 \\
\hline 300.518 & 0 & 0 & 0 & 0 & 0 & 0 & 0 & 0 & 0.546 \\
\hline 344.206 & 0 & 0 & 0 & 0 & 0 & 0 & 0 & 0 & 0 \\
\hline 394.244 & 0 & 0 & 0 & 0 & 0 & 0 & 0 & 0 & 0 \\
\hline 451.556 & 0 & 0 & 0 & 0 & 0 & 0 & 0 & 0 & 0 \\
\hline 517.2 & 0 & 0 & 0 & 0 & 0 & 0 & 0 & 0 & 0 \\
\hline 592.387 & 0 & 0 & 0 & 0 & 0 & 0 & 0 & 0 & 0 \\
\hline
\end{tabular}




\begin{tabular}{|c|c|c|c|}
\hline $\begin{array}{l}\text { Filename } \\
\text { ID\# }\end{array}$ & $\begin{array}{l}: 9911082 W M 182 \# 3 A-01 \text { Set Vel } 1 \text { min } \\
: 200002080935085\end{array}$ & $\begin{array}{l}\text { Filename } \\
\text { ID\# }\end{array}$ & $\begin{array}{l}\text { :9911082WM182\#3A-01 Set Vel } 19 \mathrm{~min} \\
: 200002080953086\end{array}$ \\
\hline Circulation $\varsigma$ & peed $: 5$ & Circulation & oeed $: 5$ \\
\hline Ultra sonic & $: 00: 21$ & Ultra sonic & $: 00: 21$ \\
\hline |Laser T\% & $: 84.2(\%)$ & |Laser T\% & $: 88.5(\%)$ \\
\hline IForm of Dist & bution:Standard & IForm of Dist & ibution:Standard \\
\hline ICalc. Level & $: 30$ & ICalc. Level & $: 30$ \\
\hline IR.R.Index & $: 1.35-0.10 \mathrm{i}$ & IR.R.Index & $: 1.35-0.10 \mathrm{i}$ \\
\hline Material & :WM182 Samp 3A & Material & :WM182 Solids Sam 3A \\
\hline Source & $:$ & Source & $:$ \\
\hline Lot Number & : & Lot Number & : \\
\hline Dispersion N & edium :RAL demin water + usonic & Dispersion $\Lambda$ & edium :RAL demin water + usonic \\
\hline IRemarks & :GMH operator & IRemarks & :GMH operators \\
\hline IRemarks 1 & :08 Feb 2000 & IRemarks 1 & :08 Feb 2000 \\
\hline IRemarks 2 & :Run \#1 & IRemarks 2 & :Run \#2 \\
\hline IMean & $: 11.140379(\mu \mathrm{m})$ & IMean & $: 17.622223(\mu \mathrm{m})$ \\
\hline Variance & :88.970901 & Variance & :643.727295 \\
\hline S.D. & $: 9.432439(\mu \mathrm{m})$ & S.D. & $: 25.371782(\mu \mathrm{m})$ \\
\hline Mode & $: 10.814984(\mu \mathrm{m})$ & Mode & : 7.215792( $(\mu \mathrm{m})$ \\
\hline Geo. Mean & $: 7.715176(\mu \mathrm{m})$ & JGeo. Mean & $-9.138258(\mu \mathrm{m})$ \\
\hline IFilename & :9911082WM182\#3A-01 Set Vel $33 \mathrm{~min}$ & IFilename & :9911082WM182\#3A-01 Set Vel $47 \mathrm{~min}$ \\
\hline liD\# & $: 200002081008087$ & IID\# & :200002081021088 \\
\hline Circulation $s$ & seed $: 5$ & Circulation $\varsigma$ & oeed $: 5$ \\
\hline Ultra sonic & $: 00: 22$ & Ultra sonic & $: 00: 20$ \\
\hline Laser T\% & : 91.6(\%) & Laser T\% & : 89.5(\%) \\
\hline jForm of Dist & bution:Standard & Form of Dist & ibution:Standard \\
\hline |Calc. Level & $: 30$ & |Calc. Level & $: 30$ \\
\hline IR.R.Index & $: 1.35-0.10 \mathrm{i}$ & IR.R.Index & $: 1.35-0.10 i$ \\
\hline IMaterial & :WM182 Solids Sam 3A & IMaterial & :WM182 Solids Sam 3A \\
\hline Source & $:$ & Source & $:$ \\
\hline Lot Number & : & Lot Number & : \\
\hline Dispersion $\mathrm{N}$ & edium :RAL demin water + usonic & Dispersion 1 & edium :RAL demin water + usonic \\
\hline Remarks & :GMH operators & Remarks & :GMH operators \\
\hline Remarks 1 & :08 Feb 2000 & |Remarks 1 & :08 Feb 2000 \\
\hline IRemarks 2 & :Run \#3 & IRemarks 2 & :Run \#4 \\
\hline IMean & $: 8.391519(\mu \mathrm{m})$ & IMean & $: 6.556015(\mu \mathrm{m})$ \\
\hline IVariance & :63.008438 & IVariance & :23.448687 \\
\hline S.D. & $: 7.937786(\mu \mathrm{m})$ & S.D. & : 4.842384( $\mu \mathrm{m})$ \\
\hline Mode & $:$ 6.293754( $\mu \mathrm{m})$ & Mode & $: 6.287407(\mu \mathrm{m})$ \\
\hline Geo. Mean & $-: 5.855623(\mu \mathrm{m})$ & JGeo. Mean & L $: 5.007885(\mu \mathrm{m})$ \\
\hline
\end{tabular}




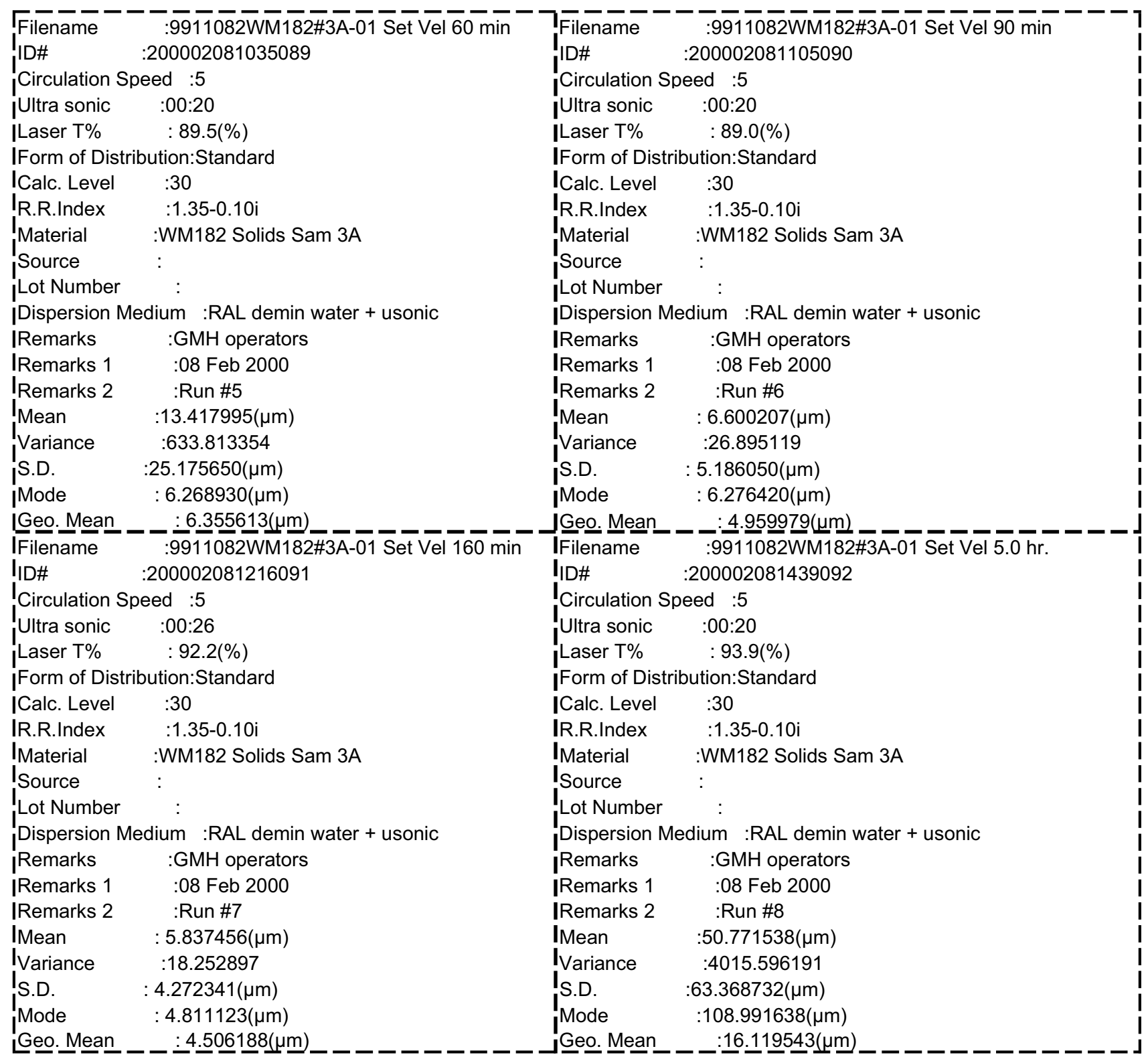




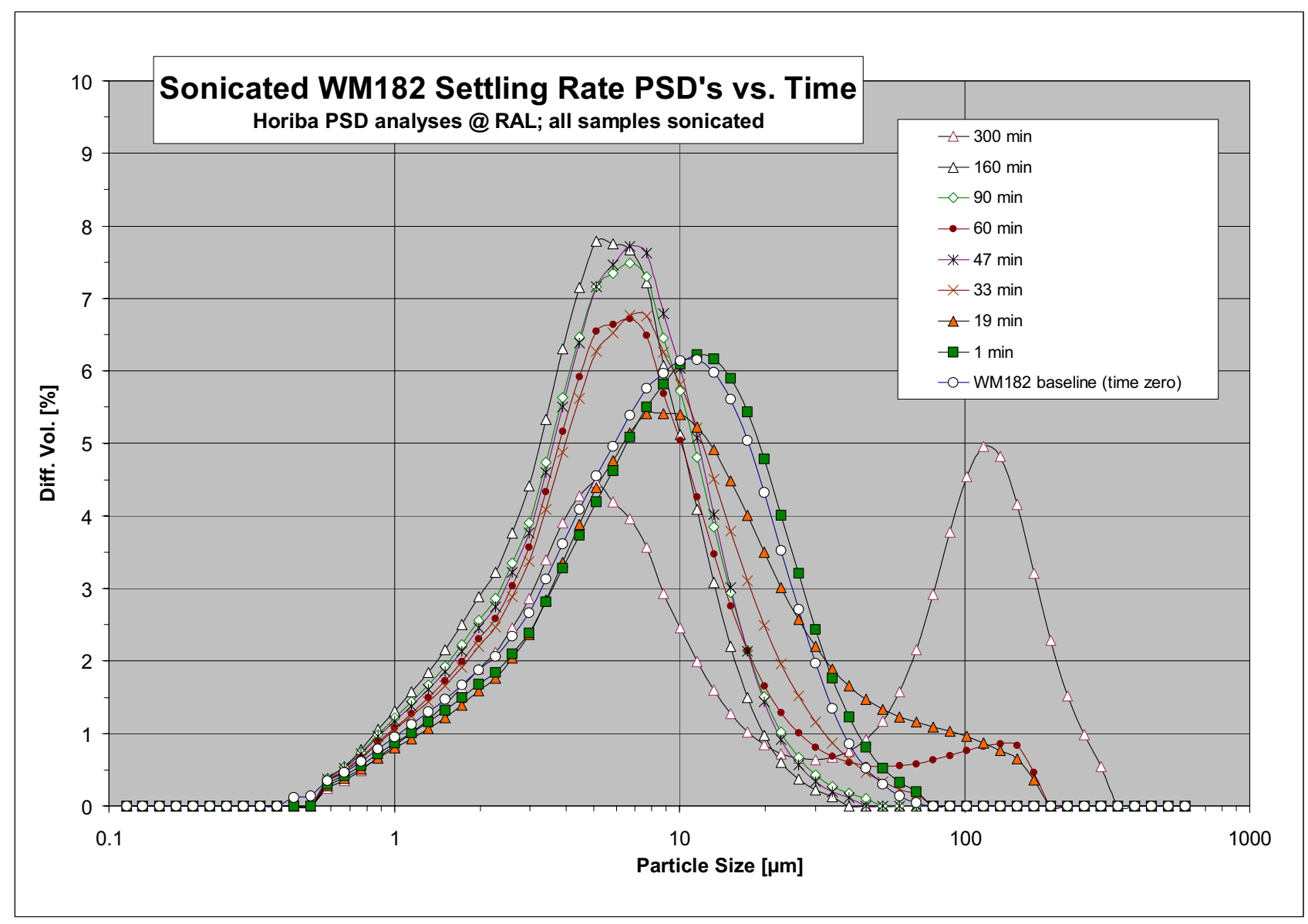


WM183 Composite A Settling Rate PSD's vs. Time

\begin{tabular}{|c|c|c|c|c|c|c|c|}
\hline \multirow[b]{2}{*}{ Time [min] } & \multirow{2}{*}{$\begin{array}{c}\text { Diameter } \\
\lceil\mu \mathrm{m}\rceil\end{array}$} & \multicolumn{6}{|c|}{ Frequency (\%) } \\
\hline & & 0 & 1 & 15 & 30 & 45 & 60 \\
\hline & 0.115 & 0 & 0 & 0 & 0 & 0 & 0 \\
\hline & 0.131 & 0 & 0 & 0 & 0 & 0 & 0 \\
\hline & 0.15 & 0 & 0 & 0 & 0 & 0 & 0 \\
\hline & 0.172 & 0 & 0 & 0 & 0 & 0 & 0 \\
\hline & 0.197 & 0 & 0 & 0 & 0 & 0 & 0 \\
\hline & 0.226 & 0 & 0 & 0 & 0 & 0 & 0 \\
\hline & 0.259 & 0 & 0 & 0 & 0 & 0 & 0 \\
\hline & 0.296 & 0 & 0 & 0 & 0 & 0 & 0 \\
\hline & 0.339 & 0 & 0 & 0 & 0 & 0 & 0 \\
\hline & 0.389 & 0 & 0 & 0 & 0 & 0 & 0 \\
\hline & 0.445 & 0 & 0 & 0 & 0 & 0 & 0 \\
\hline & 0.51 & 0 & 0 & 0 & 0 & 0 & 0 \\
\hline & 0.584 & 0 & 0.105 & 0 & 0 & 0 & 0.101 \\
\hline & 0.669 & 0 & 0.165 & 0 & 0.125 & 0.144 & 0.197 \\
\hline & 0.766 & 0.16 & 0.253 & 0.141 & 0.266 & 0.3 & 0.37 \\
\hline & 0.877 & 0.241 & 0.376 & 0.285 & 0.51 & 0.567 & 0.647 \\
\hline & 1.005 & 0.337 & 0.525 & 0.514 & 0.842 & 0.923 & 1.005 \\
\hline & 1.151 & 0.45 & 0.703 & 0.832 & 1.23 & 1.337 & 1.426 \\
\hline & 1.318 & 0.57 & 0.906 & 1.235 & 1.644 & 1.778 & 1.883 \\
\hline & 1.51 & 0.699 & 1.144 & 1.795 & 2.123 & 2.288 & 2.416 \\
\hline & 1.729 & 0.832 & 1.385 & 2.303 & 2.463 & 2.659 & 2.847 \\
\hline & 1.981 & 0.968 & 1.63 & 2.829 & 2.815 & 3.047 & 3.289 \\
\hline & 2.269 & 1.106 & 1.859 & 3.359 & 3.225 & 3.493 & 3.745 \\
\hline & 2.599 & 1.314 & 2.141 & 4.128 & 3.86 & 4.181 & 4.405 \\
\hline & 2.976 & 1.555 & 2.464 & 5.035 & 4.531 & 4.915 & 5.133 \\
\hline & 3.409 & 1.956 & 2.92 & 6.39 & 5.602 & 6.074 & 6.199 \\
\hline & 3.905 & 2.454 & 3.427 & 7.895 & 6.702 & 7.247 & 7.236 \\
\hline & 4.472 & 3.052 & 3.962 & 9.397 & 7.774 & 8.346 & 8.13 \\
\hline & 5.122 & 3.748 & 4.532 & 10.587 & 8.64 & 9.162 & 8.721 \\
\hline & 5.867 & 4.435 & 5.131 & 10.284 & 8.725 & 8.993 & 8.432 \\
\hline & 6.72 & 5.142 & 5.746 & 9.785 & 8.704 & 8.721 & 8.092 \\
\hline & 7.697 & 5.759 & 6.336 & 8.456 & 8.149 & 7.871 & 7.297 \\
\hline & 8.816 & 5.966 & 6.735 & 5.777 & 6.558 & 5.974 & 5.689 \\
\hline & 10.097 & 6.175 & 7.085 & 3.984 & 5.276 & 4.542 & 4.446 \\
\hline & 11.565 & 6.136 & 7.207 & 2.458 & 3.913 & 3.155 & 3.22 \\
\hline & 13.246 & 5.872 & 7.04 & 1.366 & 2.674 & 2.004 & 2.161 \\
\hline & 15.172 & 5.442 & 6.552 & 0.693 & 1.687 & 1.17 & 1.348 \\
\hline & 17.377 & 4.92 & 5.757 & 0.327 & 0.99 & 0.633 & 0.787 \\
\hline & 19.904 & 4.373 & 4.727 & 0.147 & 0.544 & 0.323 & 0.433 \\
\hline & 22.797 & 3.851 & 3.588 & 0 & 0.285 & 0.157 & 0.228 \\
\hline & 26.111 & 3.382 & 2.491 & 0 & 0.144 & 0 & 0.117 \\
\hline & 29.907 & 2.977 & 1.567 & 0 & 0 & 0 & 0 \\
\hline & 34.255 & 2.632 & 0.886 & 0 & 0 & 0 & 0 \\
\hline & 39.234 & 2.336 & 0.449 & 0 & 0 & 0 & 0 \\
\hline & 44.938 & 2.074 & 0.205 & 0 & 0 & 0 & 0 \\
\hline & 51.471 & 1.832 & 0 & 0 & 0 & 0 & 0 \\
\hline & 58.953 & 1.602 & 0 & 0 & 0 & 0 & 0 \\
\hline & 67.523 & 1.377 & 0 & 0 & 0 & 0 & 0 \\
\hline & 77.339 & 1.158 & 0 & 0 & 0 & 0 & 0 \\
\hline & 88.583 & 0.95 & 0 & 0 & 0 & 0 & 0 \\
\hline & 101.46 & 0.761 & 0 & 0 & 0 & 0 & 0 \\
\hline & 116.21 & 0.597 & 0 & 0 & 0 & 0 & 0 \\
\hline & 133.103 & 0.46 & 0 & 0 & 0 & 0 & 0 \\
\hline & 152.453 & 0.352 & 0 & 0 & 0 & 0 & 0 \\
\hline & 174.616 & 0 & 0 & 0 & 0 & 0 & 0 \\
\hline & 200 & 0 & 0 & 0 & 0 & 0 & 0 \\
\hline & 229.075 & 0 & 0 & 0 & 0 & 0 & 0 \\
\hline & 262.376 & 0 & 0 & 0 & 0 & 0 & 0 \\
\hline & 300.518 & 0 & 0 & 0 & 0 & 0 & 0 \\
\hline & 344.206 & 0 & 0 & 0 & 0 & 0 & 0 \\
\hline & 394.244 & 0 & 0 & 0 & 0 & 0 & 0 \\
\hline & 451.556 & 0 & 0 & 0 & 0 & 0 & 0 \\
\hline & 517.2 & 0 & 0 & 0 & 0 & 0 & 0 \\
\hline & 592.387 & 0 & 0 & 0 & 0 & 0 & 0 \\
\hline
\end{tabular}




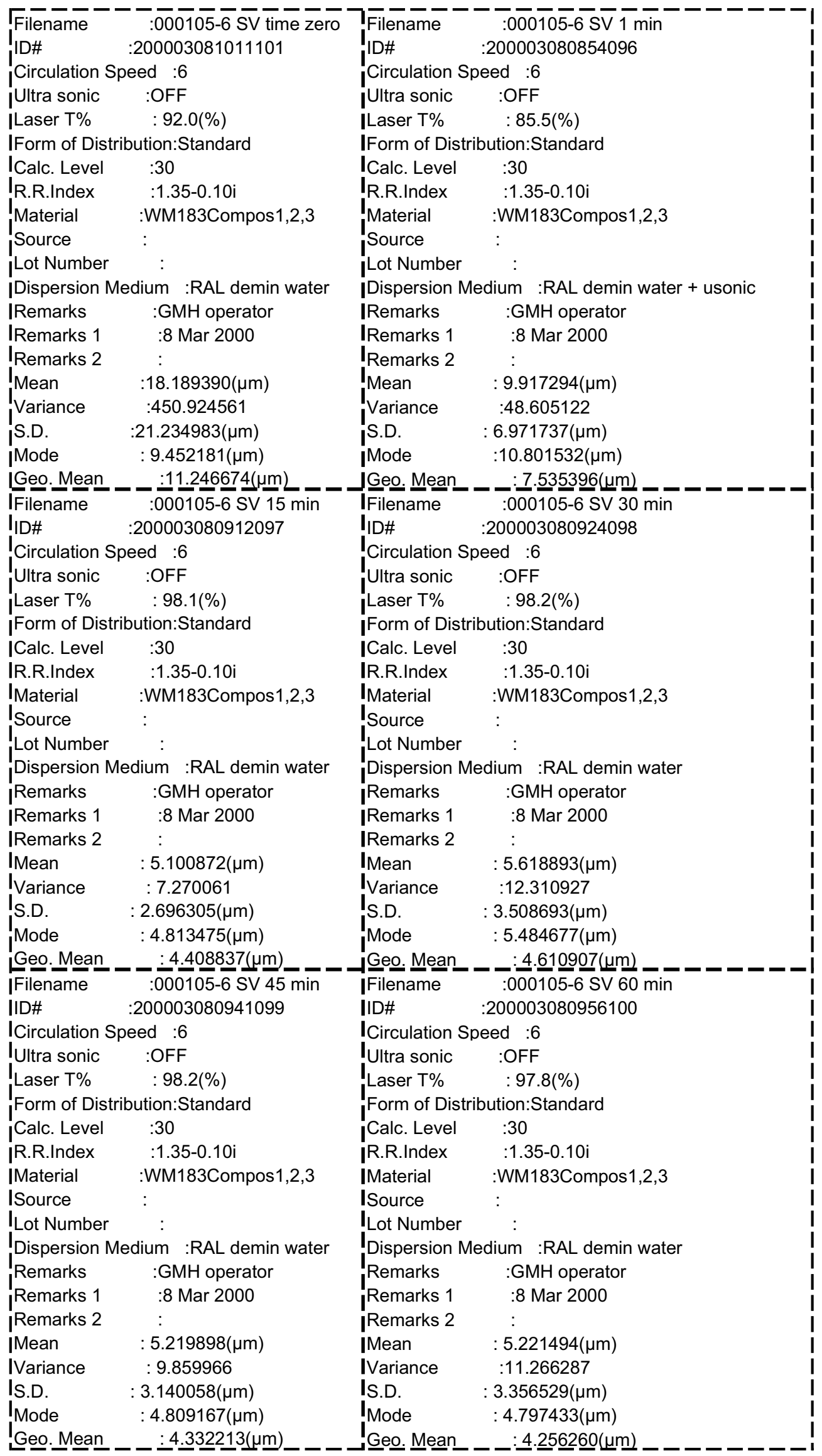




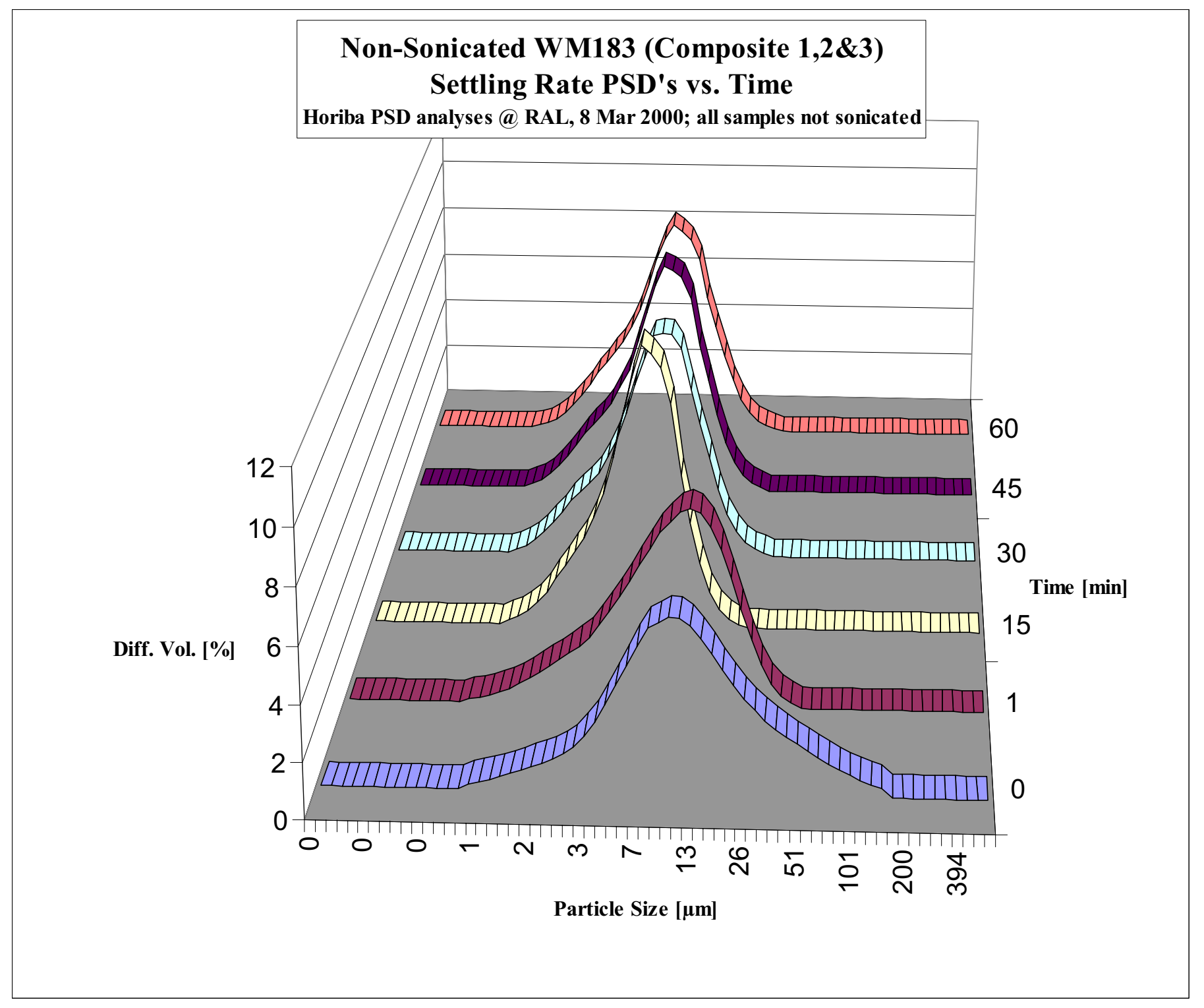


WM183 Composite A Settling Rate PSD's vs. Time

\begin{tabular}{|c|c|c|c|c|c|c|c|}
\hline \multirow[b]{2}{*}{ Time [min] } & \multirow{2}{*}{$\begin{array}{c}\text { Diameter } \\
\lceil\mu \mathrm{m}\rceil\end{array}$} & \multicolumn{6}{|c|}{ Frequency (\%) } \\
\hline & & 0 & 1 & 15 & 30 & 45 & 60 \\
\hline & 0.115 & 0 & 0 & 0 & 0 & 0 & 0 \\
\hline & 0.131 & 0 & 0 & 0 & 0 & 0 & 0 \\
\hline & 0.15 & 0 & 0 & 0 & 0 & 0 & 0 \\
\hline & 0.172 & 0 & 0 & 0 & 0 & 0 & 0 \\
\hline & 0.197 & 0 & 0 & 0 & 0 & 0 & 0 \\
\hline & 0.226 & 0 & 0 & 0 & 0 & 0 & 0 \\
\hline & 0.259 & 0 & 0 & 0 & 0 & 0 & 0 \\
\hline & 0.296 & 0 & 0 & 0 & 0 & 0 & 0 \\
\hline & 0.339 & 0 & 0 & 0 & 0 & 0 & 0 \\
\hline & 0.389 & 0 & 0 & 0 & 0 & 0 & 0 \\
\hline & 0.445 & 0 & 0 & 0 & 0 & 0 & 0 \\
\hline & 0.51 & 0 & 0 & 0 & 0 & 0 & 0 \\
\hline & 0.584 & 0 & 0.105 & 0 & 0 & 0 & 0.101 \\
\hline & 0.669 & 0 & 0.165 & 0 & 0.125 & 0.144 & 0.197 \\
\hline & 0.766 & 0.16 & 0.253 & 0.141 & 0.266 & 0.3 & 0.37 \\
\hline & 0.877 & 0.241 & 0.376 & 0.285 & 0.51 & 0.567 & 0.647 \\
\hline & 1.005 & 0.337 & 0.525 & 0.514 & 0.842 & 0.923 & 1.005 \\
\hline & 1.151 & 0.45 & 0.703 & 0.832 & 1.23 & 1.337 & 1.426 \\
\hline & 1.318 & 0.57 & 0.906 & 1.235 & 1.644 & 1.778 & 1.883 \\
\hline & 1.51 & 0.699 & 1.144 & 1.795 & 2.123 & 2.288 & 2.416 \\
\hline & 1.729 & 0.832 & 1.385 & 2.303 & 2.463 & 2.659 & 2.847 \\
\hline & 1.981 & 0.968 & 1.63 & 2.829 & 2.815 & 3.047 & 3.289 \\
\hline & 2.269 & 1.106 & 1.859 & 3.359 & 3.225 & 3.493 & 3.745 \\
\hline & 2.599 & 1.314 & 2.141 & 4.128 & 3.86 & 4.181 & 4.405 \\
\hline & 2.976 & 1.555 & 2.464 & 5.035 & 4.531 & 4.915 & 5.133 \\
\hline & 3.409 & 1.956 & 2.92 & 6.39 & 5.602 & 6.074 & 6.199 \\
\hline & 3.905 & 2.454 & 3.427 & 7.895 & 6.702 & 7.247 & 7.236 \\
\hline & 4.472 & 3.052 & 3.962 & 9.397 & 7.774 & 8.346 & 8.13 \\
\hline & 5.122 & 3.748 & 4.532 & 10.587 & 8.64 & 9.162 & 8.721 \\
\hline & 5.867 & 4.435 & 5.131 & 10.284 & 8.725 & 8.993 & 8.432 \\
\hline & 6.72 & 5.142 & 5.746 & 9.785 & 8.704 & 8.721 & 8.092 \\
\hline & 7.697 & 5.759 & 6.336 & 8.456 & 8.149 & 7.871 & 7.297 \\
\hline & 8.816 & 5.966 & 6.735 & 5.777 & 6.558 & 5.974 & 5.689 \\
\hline & 10.097 & 6.175 & 7.085 & 3.984 & 5.276 & 4.542 & 4.446 \\
\hline & 11.565 & 6.136 & 7.207 & 2.458 & 3.913 & 3.155 & 3.22 \\
\hline & 13.246 & 5.872 & 7.04 & 1.366 & 2.674 & 2.004 & 2.161 \\
\hline & 15.172 & 5.442 & 6.552 & 0.693 & 1.687 & 1.17 & 1.348 \\
\hline & 17.377 & 4.92 & 5.757 & 0.327 & 0.99 & 0.633 & 0.787 \\
\hline & 19.904 & 4.373 & 4.727 & 0.147 & 0.544 & 0.323 & 0.433 \\
\hline & 22.797 & 3.851 & 3.588 & 0 & 0.285 & 0.157 & 0.228 \\
\hline & 26.111 & 3.382 & 2.491 & 0 & 0.144 & 0 & 0.117 \\
\hline & 29.907 & 2.977 & 1.567 & 0 & 0 & 0 & 0 \\
\hline & 34.255 & 2.632 & 0.886 & 0 & 0 & 0 & 0 \\
\hline & 39.234 & 2.336 & 0.449 & 0 & 0 & 0 & 0 \\
\hline & 44.938 & 2.074 & 0.205 & 0 & 0 & 0 & 0 \\
\hline & 51.471 & 1.832 & 0 & 0 & 0 & 0 & 0 \\
\hline & 58.953 & 1.602 & 0 & 0 & 0 & 0 & 0 \\
\hline & 67.523 & 1.377 & 0 & 0 & 0 & 0 & 0 \\
\hline & 77.339 & 1.158 & 0 & 0 & 0 & 0 & 0 \\
\hline & 88.583 & 0.95 & 0 & 0 & 0 & 0 & 0 \\
\hline & 101.46 & 0.761 & 0 & 0 & 0 & 0 & 0 \\
\hline & 116.21 & 0.597 & 0 & 0 & 0 & 0 & 0 \\
\hline & 133.103 & 0.46 & 0 & 0 & 0 & 0 & 0 \\
\hline & 152.453 & 0.352 & 0 & 0 & 0 & 0 & 0 \\
\hline & 174.616 & 0 & 0 & 0 & 0 & 0 & 0 \\
\hline & 200 & 0 & 0 & 0 & 0 & 0 & 0 \\
\hline & 229.075 & 0 & 0 & 0 & 0 & 0 & 0 \\
\hline & 262.376 & 0 & 0 & 0 & 0 & 0 & 0 \\
\hline & 300.518 & 0 & 0 & 0 & 0 & 0 & 0 \\
\hline & 344.206 & 0 & 0 & 0 & 0 & 0 & 0 \\
\hline & 394.244 & 0 & 0 & 0 & 0 & 0 & 0 \\
\hline & 451.556 & 0 & 0 & 0 & 0 & 0 & 0 \\
\hline & 517.2 & 0 & 0 & 0 & 0 & 0 & 0 \\
\hline & 592.387 & 0 & 0 & 0 & 0 & 0 & 0 \\
\hline
\end{tabular}




\begin{tabular}{|c|c|c|c|}
\hline $\begin{array}{l}\text { Filename } \\
\text { ID\# }\end{array}$ & $\begin{array}{l}\text { :000105-6 SV time zero } \\
: 200003081011101\end{array}$ & $\begin{array}{l}\text { Filename } \\
\text { ID\# }\end{array}$ & $\begin{array}{l}: 000105-6 \text { SV } 1 \mathrm{~min} \\
: 200003080854096\end{array}$ \\
\hline \multicolumn{2}{|c|}{ Circulation Speed :6 } & \multicolumn{2}{|c|}{ Circulation Speed :6 } \\
\hline Ultra sonic & :OFF & Ultra sonic & :OFF \\
\hline |Laser T\% & $: 92.0(\%)$ & Laser T\% & $: 85.5(\%)$ \\
\hline \multicolumn{2}{|c|}{ IForm of Distribution:Standard } & \multicolumn{2}{|c|}{ IForm of Distribution:Standard } \\
\hline ICalc. Level & :30 & ICalc. Level & $: 30$ \\
\hline R.R.Index & $: 1.35-0.10 \mathrm{i}$ & R.R.Index & $: 1.35-0.10 \mathrm{i}$ \\
\hline Material & :WM183Compos1,2,3 & Material & :WM183Compos1,2,3 \\
\hline Source & $:$ & Source & $:$ \\
\hline Lot Number & : & Lot Number & : \\
\hline \multicolumn{2}{|c|}{ Dispersion Medium :RAL demin water } & \multicolumn{2}{|c|}{ Dispersion Medium :RAL demin water + usonic } \\
\hline IRemarks & :GMH operator & IRemarks & :GMH operator \\
\hline IRemarks 1 & :8 Mar 2000 & IRemarks 1 & :8 Mar 2000 \\
\hline IRemarks 2 & : & IRemarks 2 & : \\
\hline Mean & $: 18.189390(\mu \mathrm{m})$ & Mean & : $9.917294(\mu \mathrm{m})$ \\
\hline Variance & $: 450.924561$ & Variance & $: 48.605122$ \\
\hline S.D. & $: 21.234983(\mu \mathrm{m})$ & S.D. & : 6.971737( $\mu \mathrm{m})$ \\
\hline Mode & $: 9.452181(\mu \mathrm{m})$ & Mode & $: 10.801532(\mu \mathrm{m})$ \\
\hline \multicolumn{2}{|c|}{ IGeo. Mean _ : $: 11.246674(\mu \mathrm{m})$} & \multicolumn{2}{|c|}{ JGeo. Mean - } \\
\hline IFilename & $: 000105-6$ SV 15 min & IFilename & $: 000105-6$ SV 30 min \\
\hline lID\# & :200003080912097 & IID\# & $: 200003080924098$ \\
\hline \multicolumn{2}{|c|}{ ICirculation Speed :6 } & \multicolumn{2}{|c|}{ Circulation Speed :6 } \\
\hline Ultra sonic & :OFF & Ultra sonic & :OFF \\
\hline Laser T\% & : 98.1(\%) & Laser T\% & $: 98.2(\%)$ \\
\hline \multicolumn{2}{|c|}{ Form of Distribution:Standard } & \multicolumn{2}{|c|}{ Form of Distribution:Standard } \\
\hline |Calc. Level & $: 30$ & ICalc. Level & $: 30$ \\
\hline IR.R.Index & $: 1.35-0.10 i$ & IR.R.Index & $: 1.35-0.10 \mathrm{i}$ \\
\hline IMaterial & :WM183Compos1,2,3 & IMaterial & :WM183Compos1,2,3 \\
\hline Source & $:$ & ISource & $:$ \\
\hline Lot Number & : & Lot Number & : \\
\hline \multicolumn{2}{|c|}{ Dispersion Medium :RAL demin water } & \multicolumn{2}{|c|}{ Dispersion Medium :RAL demin water } \\
\hline Remarks & :GMH operator & Remarks & :GMH operator \\
\hline |Remarks 1 & :8 Mar 2000 & IRemarks 1 & :8 Mar 2000 \\
\hline IRemarks 2 & : & IRemarks 2 & : \\
\hline IMean & $: 5.100872(\mu \mathrm{m})$ & IMean & $: 5.618893(\mu \mathrm{m})$ \\
\hline Variance & $: 7.270061$ & IVariance & :12.310927 \\
\hline S.D. & : 2.696305( $\mu \mathrm{m})$ & S.D. & : 3.508693( $\mu \mathrm{m})$ \\
\hline Mode & $: 4.813475(\mu \mathrm{m})$ & Mode & $: 5.484677(\mu \mathrm{m})$ \\
\hline \multicolumn{2}{|c|}{ 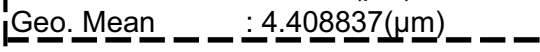 } & \multicolumn{2}{|c|}{ Geo. Mean _: $4.610907(\mu \mathrm{m})$} \\
\hline Filename & :000105-6 SV $45 \mathrm{~min}$ & Filename & :000105-6 SV $60 \mathrm{~min}$ \\
\hline IID\# & :200003080941099 & IID\# & $: 200003080956100$ \\
\hline \multicolumn{2}{|c|}{ ICirculation Speed :6 } & \multicolumn{2}{|c|}{ ICirculation Speed :6 } \\
\hline lUltra sonic & :OFF & IUltra sonic & :OFF \\
\hline Laser T\% & : 98.2(\%) & Laser T\% & $: 97.8(\%)$ \\
\hline Form of Dist & ibution:Standard & Form of Dist & ibution:Standard \\
\hline Calc. Level & $: 30$ & Calc. Level & $: 30$ \\
\hline R.R.Index & $: 1.35-0.10 \mathrm{i}$ & R.R.Index & $: 1.35-0.10 \mathrm{i}$ \\
\hline |Material & :WM183Compos1,2,3 & IMaterial & :WM183Compos1,2,3 \\
\hline ISource & $:$ & ISource & : \\
\hline Lot Number & : & Lot Number & : \\
\hline Dispersion $\mathrm{N}$ & ledium :RAL demin water & Dispersion 1 & edium $:$ RAL demin water \\
\hline Remarks & :GMH operator & Remarks & :GMH operator \\
\hline Remarks 1 & :8 Mar 2000 & Remarks 1 & :8 Mar 2000 \\
\hline Remarks 2 & : & Remarks 2 & : \\
\hline |Mean & $: 5.219898(\mu \mathrm{m})$ & IMean & $: 5.221494(\mu \mathrm{m})$ \\
\hline IVariance & : 9.859966 & IVariance & :11.266287 \\
\hline IS.D. & $: 3.140058(\mu \mathrm{m})$ & IS.D. & $: 3.356529(\mu \mathrm{m})$ \\
\hline Mode & : 4.809167( $\mu \mathrm{m})$ & IMode & : 4.797433( $\mu \mathrm{m})$ \\
\hline Geo. Mean & $: 4.332213(\mu \mathrm{m})$ & Geo. Mean & - _: $\underline{4} . \underline{25} \underline{6} \underline{60}(\underline{\mu} \underline{m})$ \\
\hline
\end{tabular}




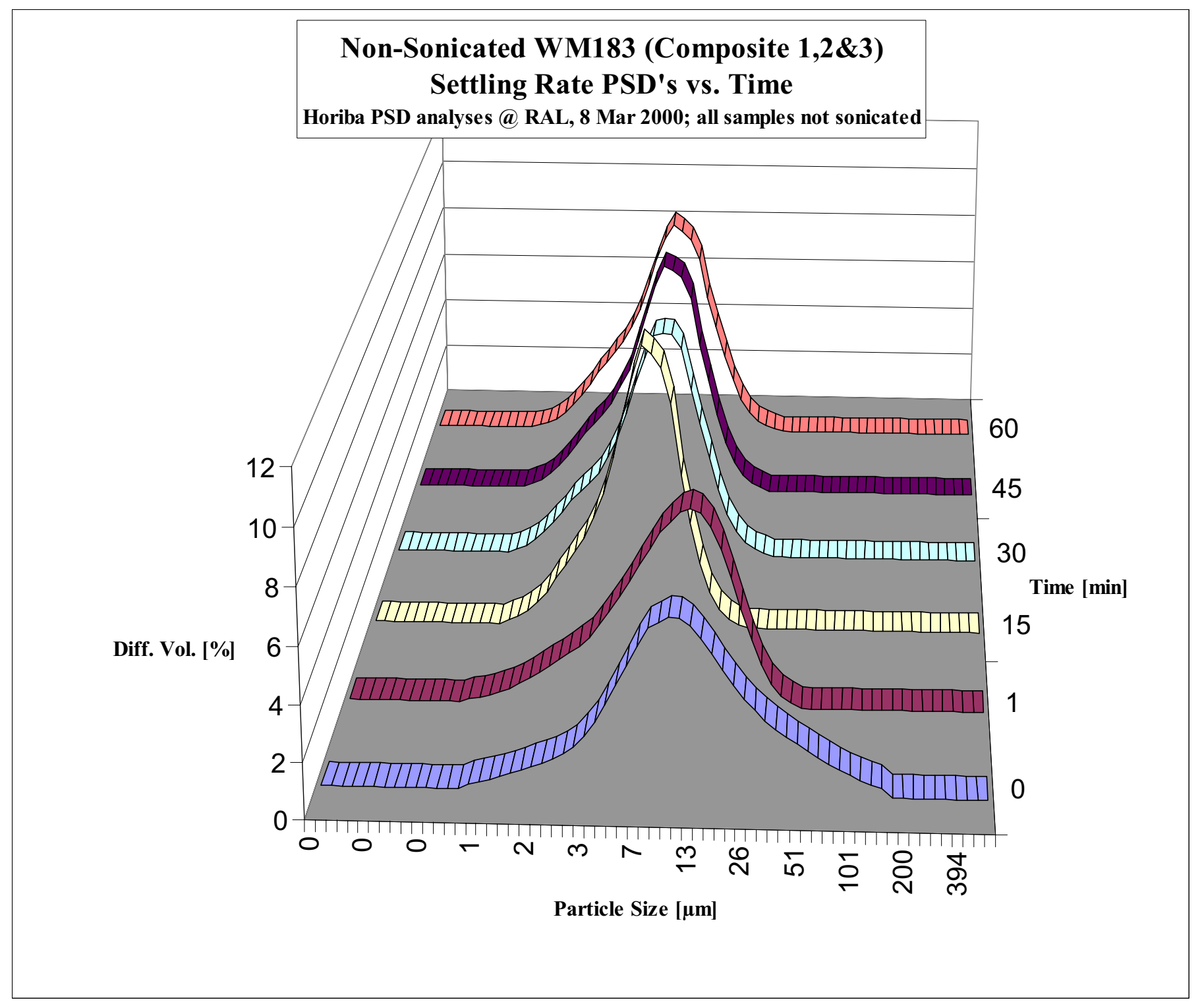




\section{Non-Sonicated WM183 Composite B Settling Rate PSD's vs. Time \\ 8 Mar 2000}

\begin{tabular}{|c|c|c|c|c|c|c|c|}
\hline \multirow[b]{2}{*}{ Time [min] } & \multirow{2}{*}{$\begin{array}{c}\text { Diameter } \\
\qquad \mu \mathrm{m}\rceil\end{array}$} & \multicolumn{6}{|c|}{ Frequency (\%) } \\
\hline & & 0 & 1 & 15 & 30 & 45 & 60 \\
\hline & 0.115 & 0 & 0 & 0 & 0 & 0 & 0 \\
\hline & 0.131 & 0 & 0 & 0 & 0 & 0 & 0 \\
\hline & 0.15 & 0 & 0 & 0 & 0 & 0 & 0 \\
\hline & 0.172 & 0 & 0 & 0 & 0 & 0 & 0 \\
\hline & 0.197 & 0 & 0 & 0 & 0 & 0 & 0 \\
\hline & 0.226 & 0 & 0 & 0 & 0 & 0 & 0 \\
\hline & 0.259 & 0 & 0 & 0 & 0 & 0 & 0 \\
\hline & 0.296 & 0 & 0 & 0 & 0 & 0 & 0 \\
\hline & 0.339 & 0 & 0 & 0 & 0 & 0 & 0 \\
\hline & 0.389 & 0 & 0 & 0 & 0 & 0 & 0 \\
\hline & 0.445 & 0 & 0 & 0 & 0 & 0 & 0 \\
\hline & 0.51 & 0 & 0 & 0 & 0 & 0 & 0 \\
\hline & 0.584 & 0 & 0 & 0 & 0 & 0 & 0 \\
\hline & 0.669 & 0 & 0 & 0.17 & 0.156 & 0.136 & 0.166 \\
\hline & 0.766 & 0.101 & 0.104 & 0.304 & 0.317 & 0.294 & 0.332 \\
\hline & 0.877 & 0.157 & 0.159 & 0.511 & 0.589 & 0.579 & 0.618 \\
\hline & 1.005 & 0.227 & 0.228 & 0.768 & 0.95 & 0.984 & 1.013 \\
\hline & 1.151 & 0.313 & 0.312 & 1.063 & 1.374 & 1.479 & 1.499 \\
\hline & 1.318 & 0.41 & 0.407 & 1.38 & 1.828 & 2.017 & 2.036 \\
\hline & 1.51 & 0.524 & 0.516 & 1.757 & 2.368 & 2.656 & 2.679 \\
\hline & 1.729 & 0.639 & 0.627 & 2.061 & 2.763 & 3.079 & 3.147 \\
\hline & 1.981 & 0.757 & 0.742 & 2.353 & 3.147 & 3.472 & 3.583 \\
\hline & 2.269 & 0.878 & 0.859 & 2.645 & 3.561 & 3.9 & 4.026 \\
\hline & 2.599 & 1.035 & 1.018 & 3.015 & 4.142 & 4.494 & 4.591 \\
\hline & 2.976 & 1.215 & 1.2 & 3.422 & 4.77 & 5.115 & 5.202 \\
\hline & 3.409 & 1.484 & 1.479 & 3.982 & 5.681 & 6.042 & 6.038 \\
\hline & 3.905 & 1.796 & 1.808 & 4.518 & 6.551 & 6.911 & 6.774 \\
\hline & 4.472 & 2.143 & 2.183 & 5 & 7.329 & 7.689 & 7.364 \\
\hline & 5.122 & 2.519 & 2.597 & 5.408 & 7.919 & 8.251 & 7.731 \\
\hline & 5.867 & 2.881 & 2.998 & 5.683 & 7.994 & 8.19 & 7.591 \\
\hline & 6.72 & 3.259 & 3.418 & 5.958 & 7.984 & 8.017 & 7.395 \\
\hline & 7.697 & 3.607 & 3.804 & 6.139 & 7.591 & 7.411 & 6.89 \\
\hline & 8.816 & 3.825 & 4.034 & 6.12 & 6.371 & 5.918 & 5.755 \\
\hline & 10.097 & 4.054 & 4.277 & 6.07 & 5.31 & 4.702 & 4.787 \\
\hline & 11.565 & 4.2 & 4.421 & 5.861 & 4.103 & 3.432 & 3.728 \\
\hline & 13.246 & 4.264 & 4.468 & 5.485 & 2.925 & 2.295 & 2.708 \\
\hline & 15.172 & 4.261 & 4.435 & 4.945 & 1.923 & 1.408 & 1.831 \\
\hline & 17.377 & 4.21 & 4.347 & 4.264 & 1.167 & 0.796 & 1.153 \\
\hline & 19.904 & 4.131 & 4.228 & 3.487 & 0.659 & 0.42 & 0.68 \\
\hline & 22.797 & 4.046 & 4.1 & 2.682 & 0.35 & 0.21 & 0.379 \\
\hline & 26.111 & 3.968 & 3.981 & 1.929 & 0.178 & 0.102 & 0.202 \\
\hline & 29.907 & 3.904 & 3.878 & 1.296 & 0 & 0 & 0.105 \\
\hline & 34.255 & 3.855 & 3.792 & 0.814 & 0 & 0 & 0 \\
\hline & 39.234 & 3.811 & 3.712 & 0.483 & 0 & 0 & 0 \\
\hline & 44.938 & 3.754 & 3.624 & 0.274 & 0 & 0 & 0 \\
\hline & 51.471 & 3.665 & 3.507 & 0.151 & 0 & 0 & 0 \\
\hline & 58.953 & 3.52 & 3.34 & 0 & 0 & 0 & 0 \\
\hline & 67.523 & 3.304 & 3.109 & 0 & 0 & 0 & 0 \\
\hline & 77.339 & 3.011 & 2.812 & 0 & 0 & 0 & 0 \\
\hline & 88.583 & 2.651 & 2.46 & 0 & 0 & 0 & 0 \\
\hline & 101.46 & 2.25 & 2.077 & 0 & 0 & 0 & 0 \\
\hline & 116.21 & 1.84 & 1.692 & 0 & 0 & 0 & 0 \\
\hline & 133.103 & 1.452 & 1.335 & 0 & 0 & 0 & 0 \\
\hline & 152.453 & 1.114 & 1.025 & 0 & 0 & 0 & 0 \\
\hline & 174.616 & 0.619 & 0.57 & 0 & 0 & 0 & 0 \\
\hline & 200 & 0.344 & 0.316 & 0 & 0 & 0 & 0 \\
\hline & 229.075 & 0 & 0 & 0 & 0 & 0 & 0 \\
\hline & 262.376 & 0 & 0 & 0 & 0 & 0 & 0 \\
\hline & 300.518 & 0 & 0 & 0 & 0 & 0 & 0 \\
\hline & 344.206 & 0 & 0 & 0 & 0 & 0 & 0 \\
\hline & 394.244 & 0 & 0 & 0 & 0 & 0 & 0 \\
\hline & 451.556 & 0 & 0 & 0 & 0 & 0 & 0 \\
\hline
\end{tabular}




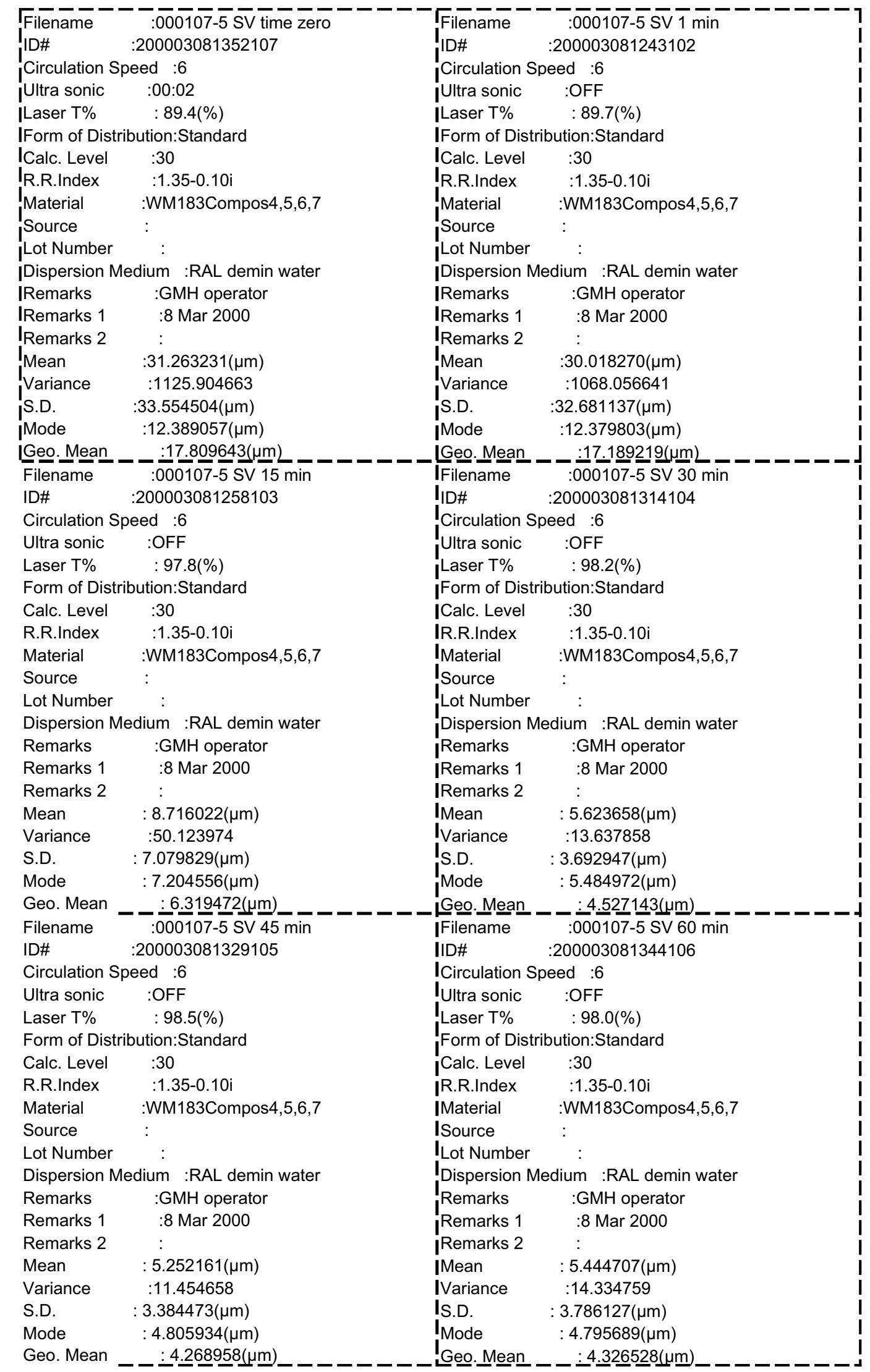




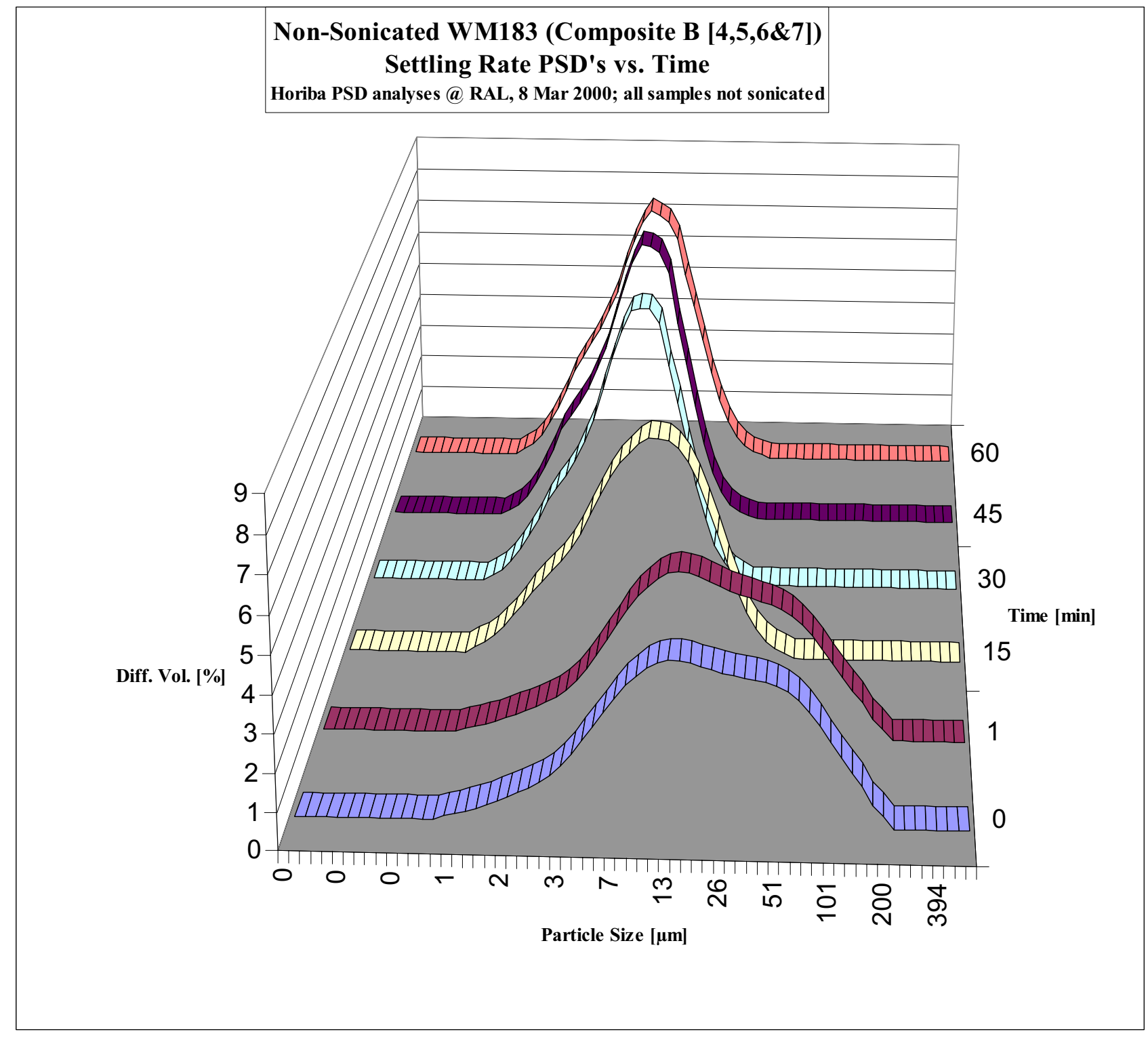




\section{Appendix A-3 \\ Settling Rate Testing Photographs}
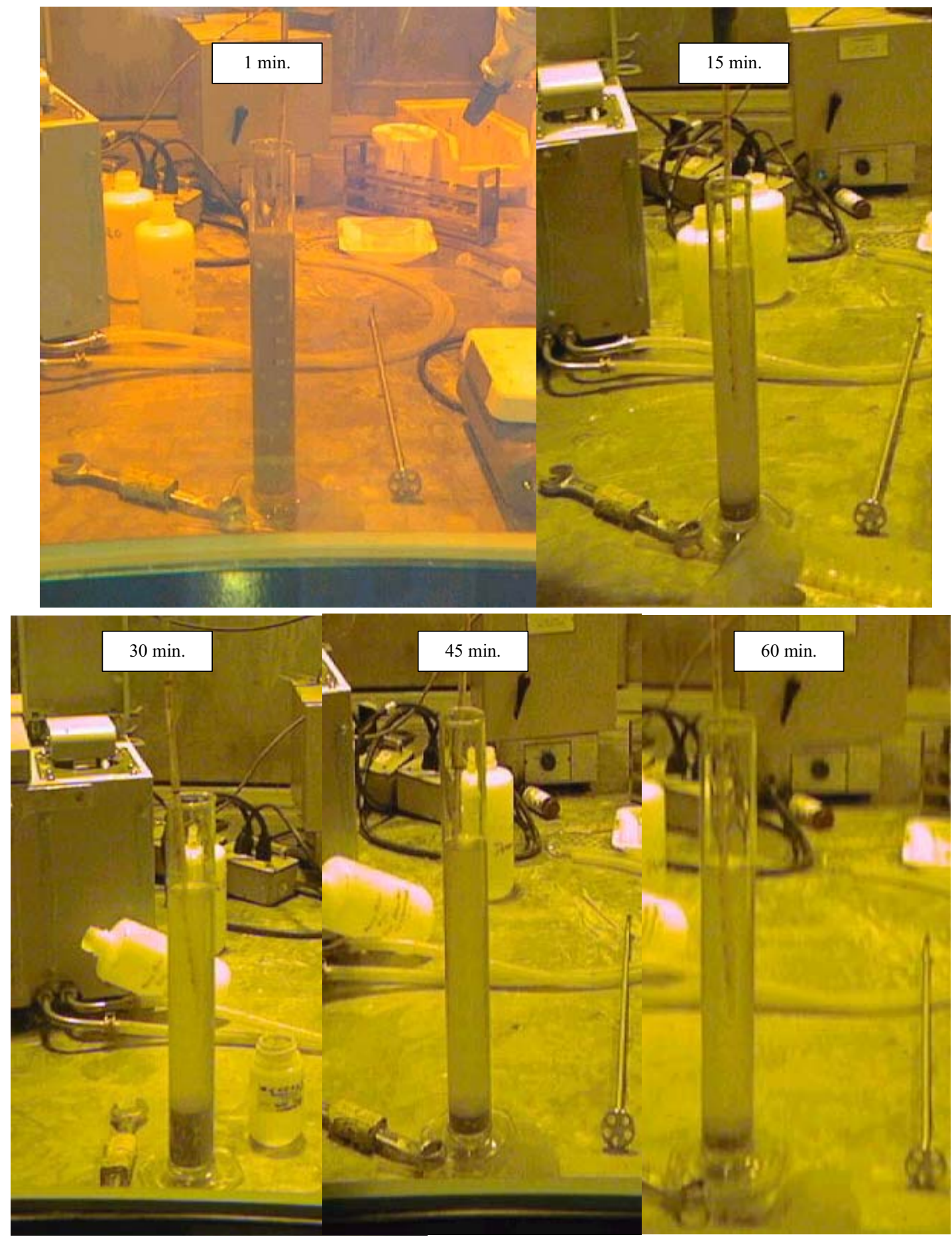

Figure A-3a. WM-183 Composite A settling rate testing photographs. 


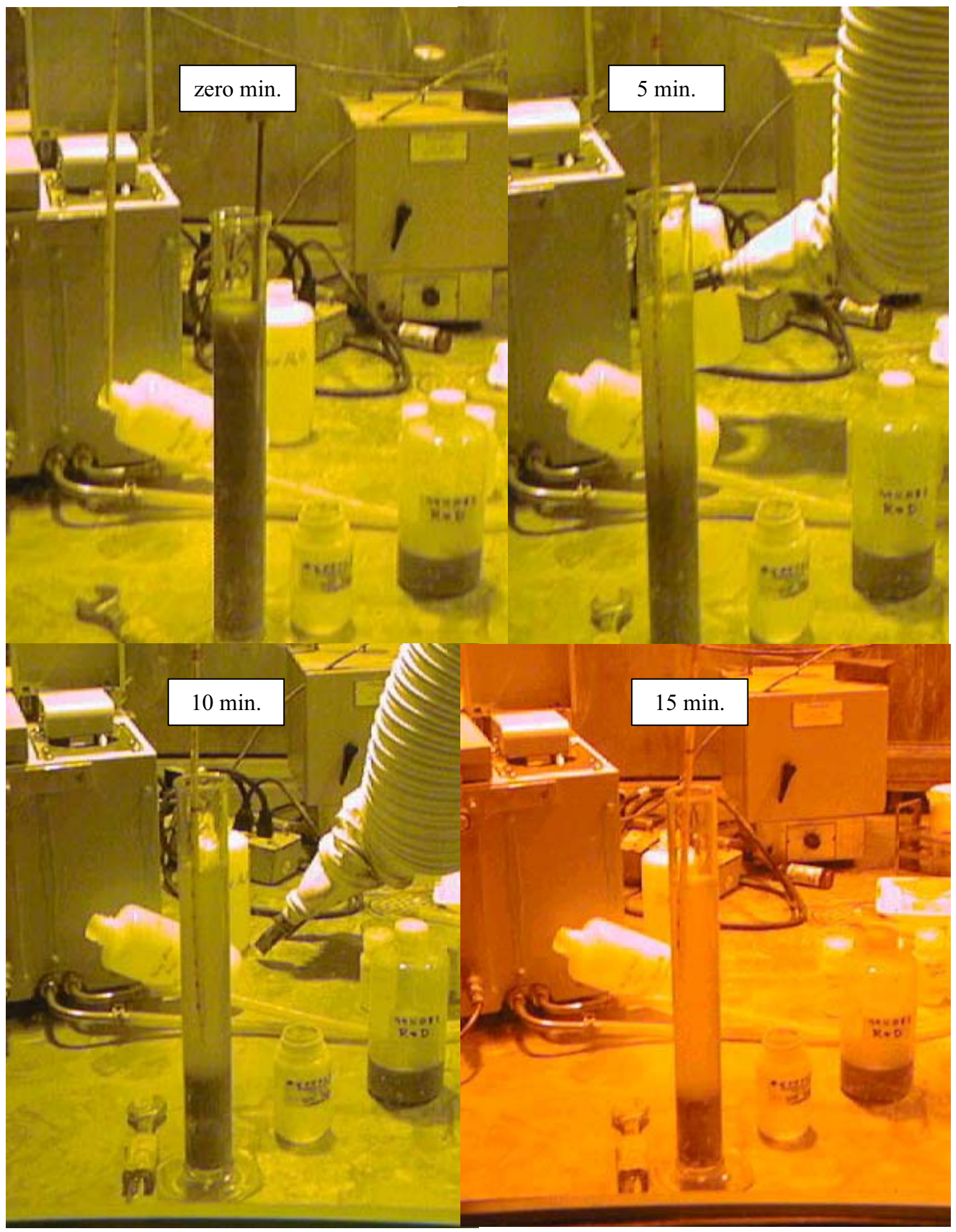

Figure A-3b. WM-183 Composite B settling rate testing photographs. 


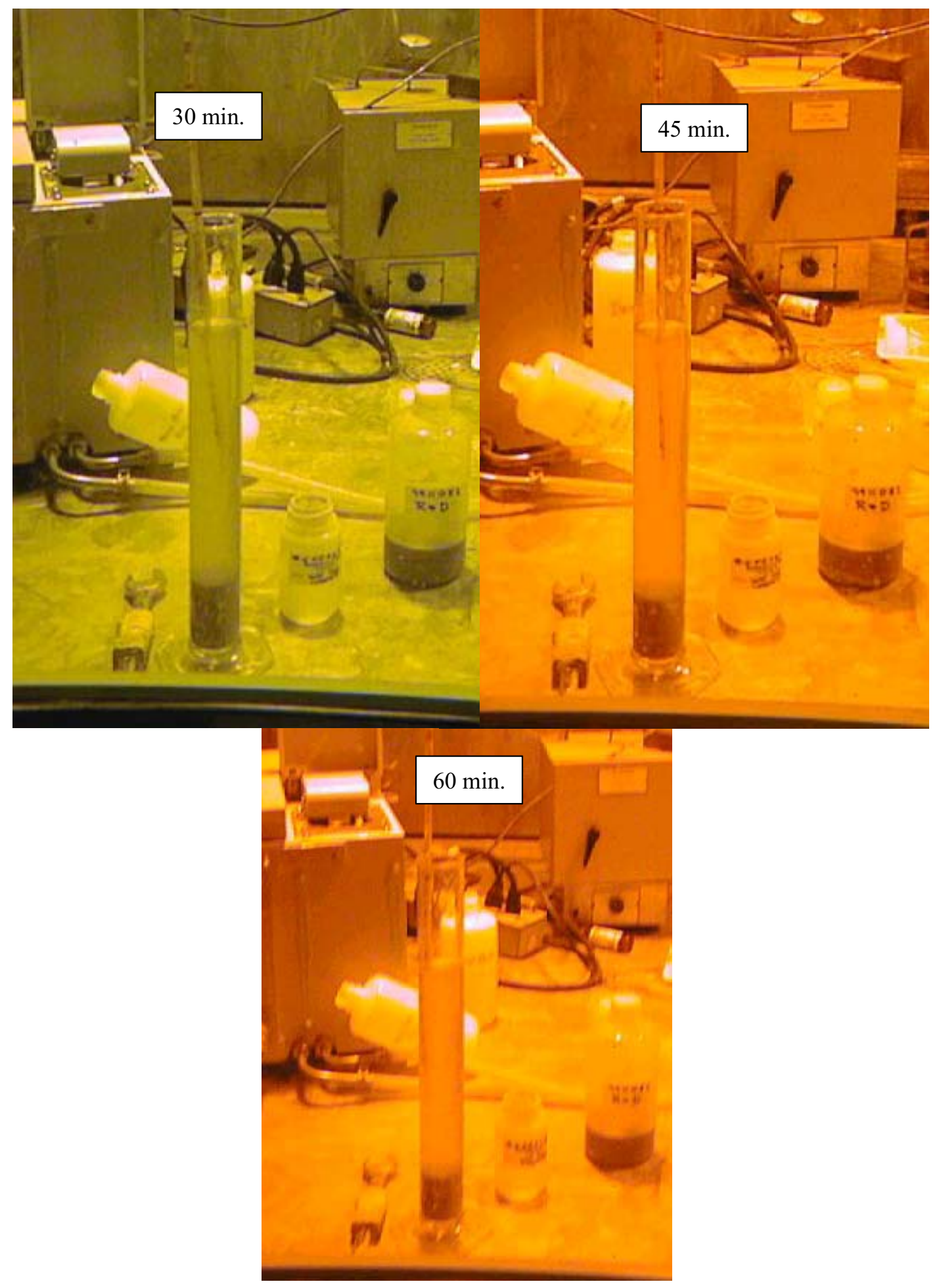

Figure A-3b. (continued). WM-183 Composite B settling rate testing photographs. 